\title{
A Solution for Overcoming Transradial Prosthetic Control Limitations with Additive Manufacturing and Modeling Techniques
}

\author{
Olivia Layne Santee
}

West Virginia University, olsantee@mix.wvu.edu

Follow this and additional works at: https://researchrepository.wvu.edu/etd

Part of the Biomechanical Engineering Commons

\section{Recommended Citation}

Santee, Olivia Layne, "A Solution for Overcoming Transradial Prosthetic Control Limitations with Additive Manufacturing and Modeling Techniques" (2021). Graduate Theses, Dissertations, and Problem Reports. 10165.

https://researchrepository.wvu.edu/etd/10165

This Thesis is protected by copyright and/or related rights. It has been brought to you by the The Research Repository @ WVU with permission from the rights-holder(s). You are free to use this Thesis in any way that is permitted by the copyright and related rights legislation that applies to your use. For other uses you must obtain permission from the rights-holder(s) directly, unless additional rights are indicated by a Creative Commons license in the record and/ or on the work itself. This Thesis has been accepted for inclusion in WVU Graduate Theses, Dissertations, and Problem Reports collection by an authorized administrator of The Research Repository @ WVU. For more information, please contact researchrepository@mail.wvu.edu. 


\section{A Solution for Overcoming Transradial Prosthetic Control Limitations with Additive Manufacturing and Modeling Techniques}

Olivia Layne Santee

Follow this and additional works at: https://researchrepository.wvu.edu/etd

Part of the Biomechanical Engineering Commons 


\title{
A Solution for Overcoming Transradial Prosthetic Control Limitations with Additive Manufacturing and Modeling Techniques
}

\author{
Olivia Layne Santee \\ Thesis submitted to the Mechanical and Aerospace Department at West Virginia University \\ In partial fulfillment of the requirements for the degree of \\ Master of Science in Mechanical Engineering
}

Sergiy Yakovenko, Ph.D., Chair

Victor Mucino, Ph.D.

Gregory Thompson, Ph.D.

Department of Mechanical and Aerospace Engineering

Morgantown, West Virginia

2021

Keywords: 3D-printed, prosthetic, cable-driven, multi-DOF, wrist flexion-extension

Copyright 2021 Olivia Layne Santee 


\begin{abstract}
A Solution for Overcoming Transradial Prosthetic Control Limitations with Additive Manufacturing and Modeling Techniques
\end{abstract}

\author{
Olivia Layne Santee
}

Prosthetic limbs have aided in the restoration of both cosmetic and functional capabilities of the human hand; however, structural and control limitations hinder widespread adoption. One example of these limitations addressed in this study is the lack of wrist degrees of freedom (DOF) in robotic hands. Using the mechanical model-driven design, I developed a hand with cable-driven actuation of fingers and direct actuation of the wrist pronation-supination and flexion-extension DOFs. An inverse dynamic simulation determined torque requirements in common tasks manipulating a $1 \mathrm{~kg}$ mass with rotational speeds up to $60 \mathrm{deg} / \mathrm{s}$, setting a minimum limitation of $0.76 \mathrm{Nm}$ on the wrist servomotors. The performance of the manufactured robotic hand was validated using motion capture. Kinematic errors were 19 deg on average with the best and worst values spanning 2 to 55 deg (thumb CMC and third digit PIP DOFs, respectively). The execution delays computed with the cross-correlation of the command-movement relationship were 200 to $400 \mathrm{~ms}$ in 6 DOF movements. This performance was appropriate for the functional use of the device and serves as the framework for testing future controller designs. 


\section{Acknowledgements}

Over the past few years at West Virginia University, I have gained invaluable knowledge and received unconditional support.

First, I would like to thank the Department of Mechanical and Aerospace Engineering for funding my first year of graduate school. I would also like to thank the Clinical and Translational Science Institute for funding the following year and a half of graduate school. I greatly appreciated the financial support from both sources, while having the opportunity to learn new material and maintain mastery of old material outside of my thesis work.

I would like to thank Dr. Sergiy Yakovenko for his help and guidance over the past few years. I was able to grow both as an engineer and a person by working in his lab.

I would also like to thank Dr. Gregory Thompson and Dr. Victor Mucino for being on my committee and offering guidance and support when needed.

Without the support of my fellow colleagues this would not have been possible. Thank you very much for all you have done from helping with general questions, experiments in the lab, and just being there: Trevor Moon, Dr. Matthew Boots, Serhii Bahdasariants, Dr. Ariel Thomas, Dr. Russel Hardesty, Dr. Anton Sobinov, Brooklyn Metz, Emily Herrick, and Kacie O’Neil.

Although they did not work with me directly in the lab, I would like to thank Benjamin Moidel and Cortney Moidel for their help and support. Thank you to my girlfriend Jenna Bailey for supporting me through even the longest nights and more challenging tasks.

Finally, I would like to thank my family for their help and support through all my years of school. I could not have done it without my family and everyone who has helped me along the way.

I would like to give a special thank you to Bergen Cable for their donation of cables, conduit, end fittings, as well as general advice. 


\section{Table of Contents}

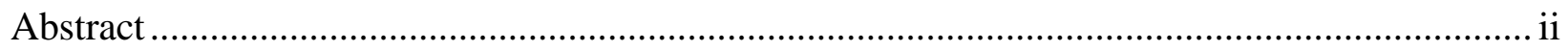

Acknowledgements ........................................................................................................ ii

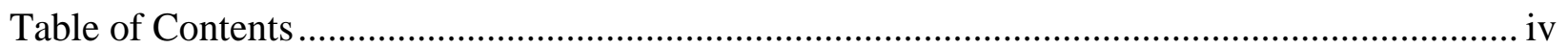

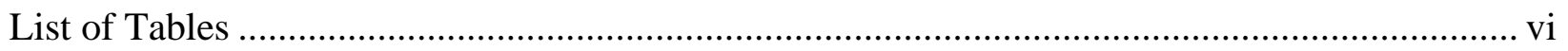

List of Figures ............................................................................................................. vii

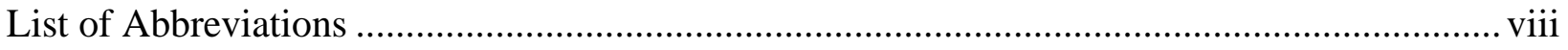

Chapter 1: Background and Literature Review …………..................................................... 1

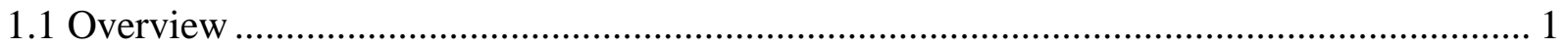

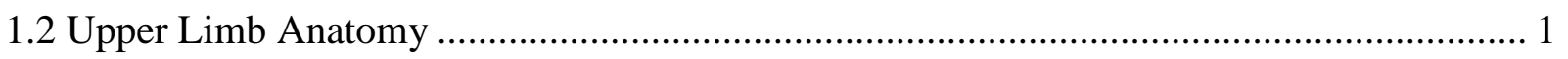

1.2.1 Hand and Forearm Anatomy ……………..................................................................... 2

1.2.2 Wrist Structure and Function.............................................................................................. 3

1.3 Mechanical Relationship to Anatomical Structure................................................................ 4

1.4 Prosthetics Review and Manufacturing Overview ................................................................. 5

1.4.1 Prosthetic Classifications and Control Techniques ...................................................... 6

1.4.2 The Balance Between Complexity and Functionality ....................................................... 9

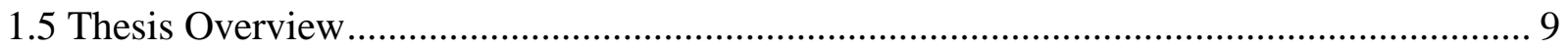

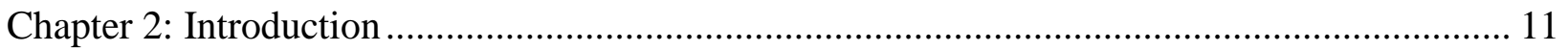

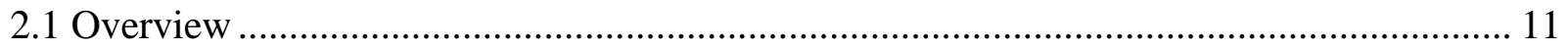

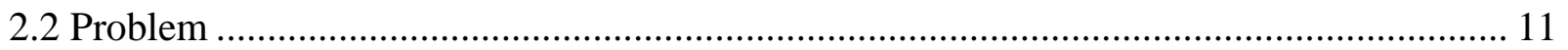

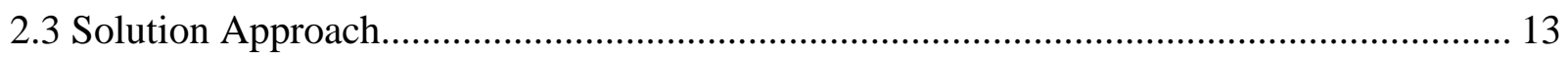

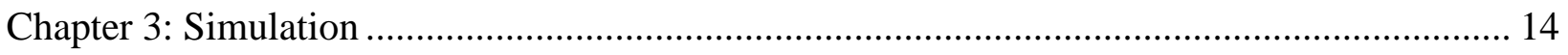

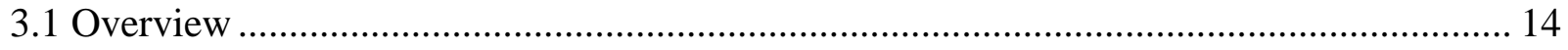

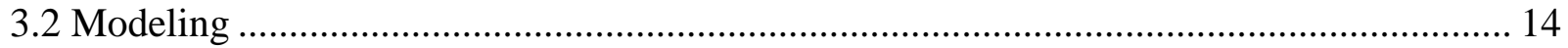

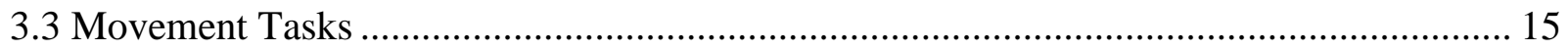

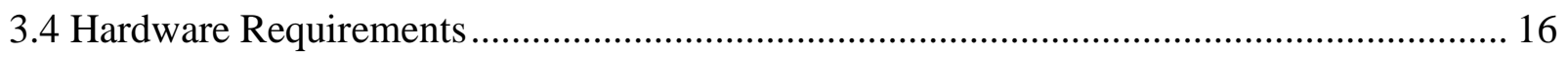

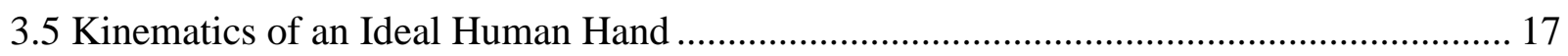

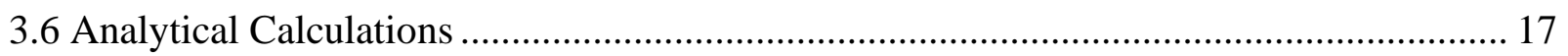

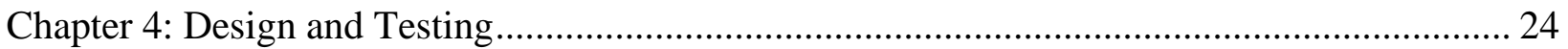

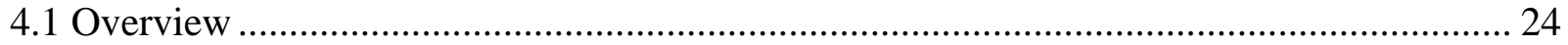

4.2 Hand Design ............................................................................................................... 24

4.3 Wrist and Forearm Design ........................................................................................ 27 


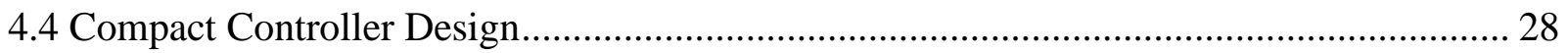

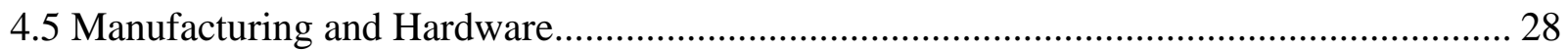

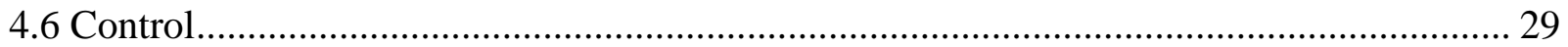

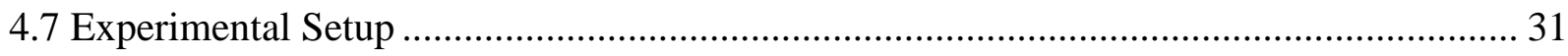

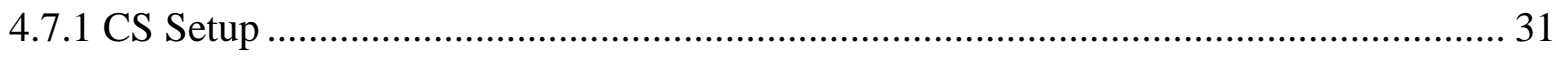

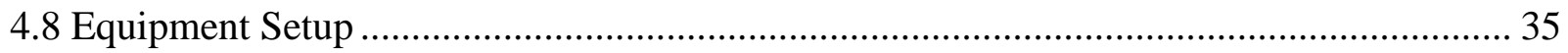

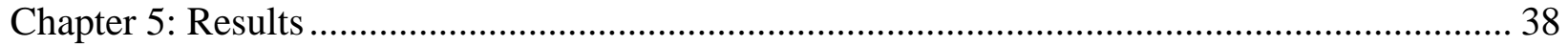

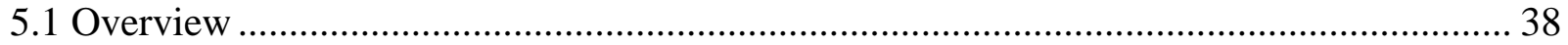

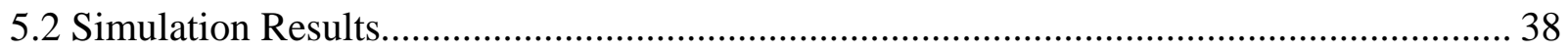

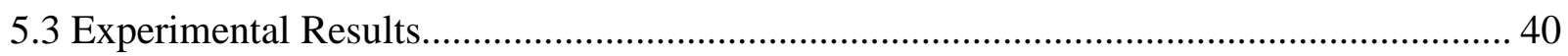

Chapter 6: Discussion and Conclusions................................................................................... 49

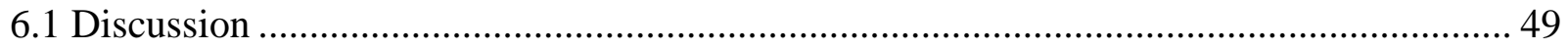

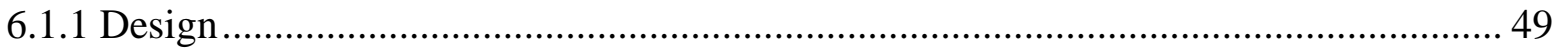

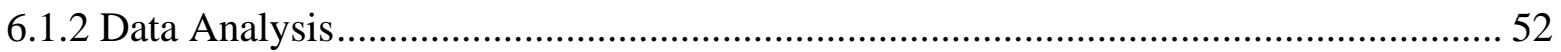

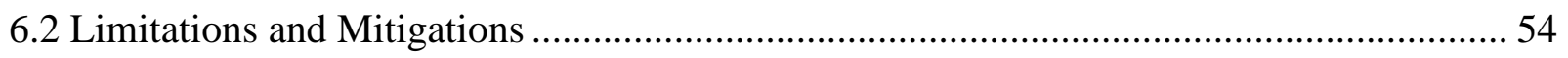

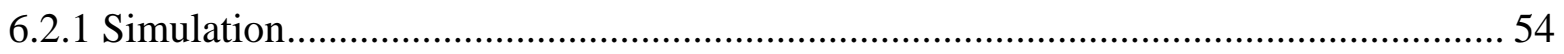

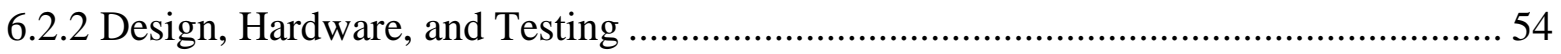

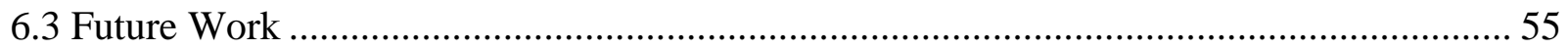

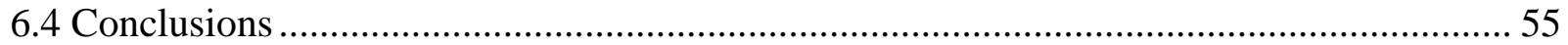

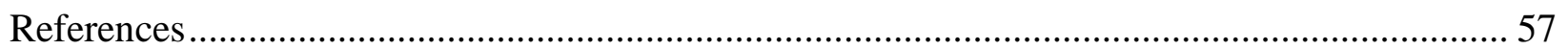

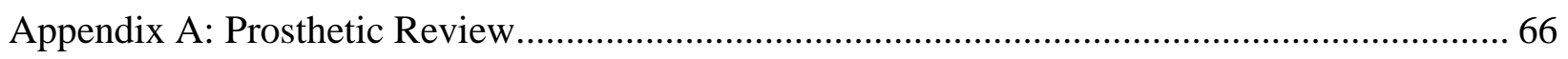

A.1: Commercial and Research Based Prosthetic Comparison ................................................. 66

This section of Appendix A contains two tables detailing the prosthetics that are commercially available as well as those that are still undergoing research...................................................... 66

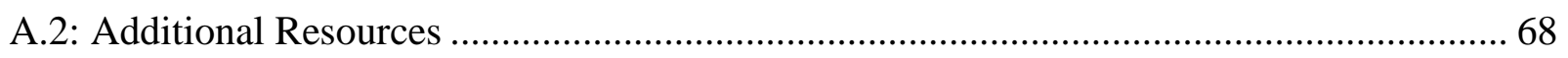

Appendix B: Method for Calculating Analytical Model Solutions ……….................................. 70

Appendix C: Establishing a Local Point in a Global CS Example .................................................. 71

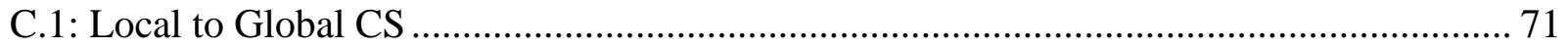

C.2: Joint CS Setup …………………………………….................................................. 71

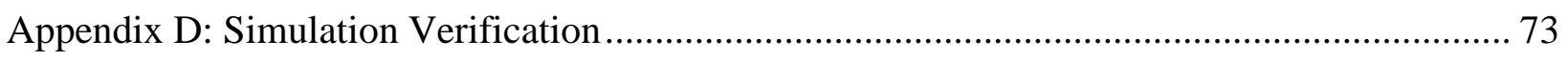




\section{List of Tables}

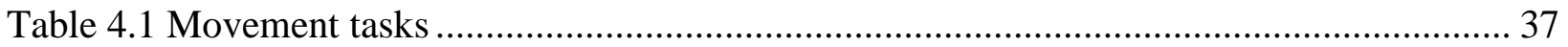

Table 5.1. Simulated torque data for hardware selection........................................................ 39

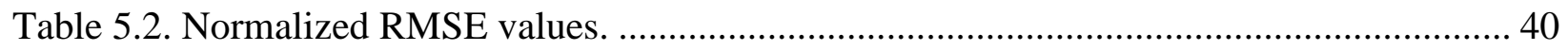

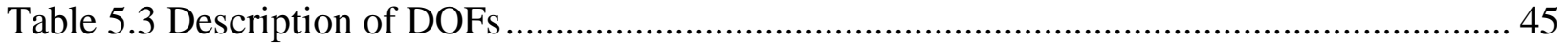




\section{List of Figures}

Figure 1.1. Upper limb anatomy................................................................................ 2

Figure 1.2. Anatomical layout of the left hand ................................................................ 2

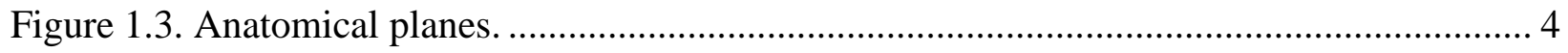

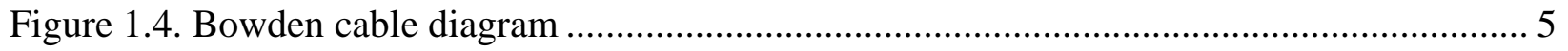

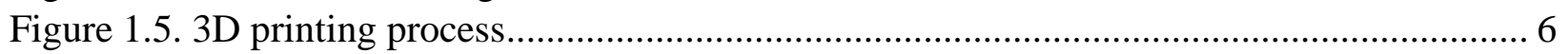

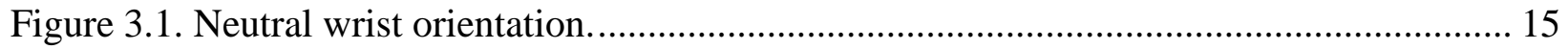

Figure 3.2. Arm postures based on the effects of gravity .................................................. 17

Figure 3.3. Diagram for analytical wrist flexion-extension calculations ................................. 19

Figure 3.4. Diagram for analytical wrist pronation calculations .......................................... 21

Figure 3.5. Diagram for analytical wrist supination calculations ........................................ 21

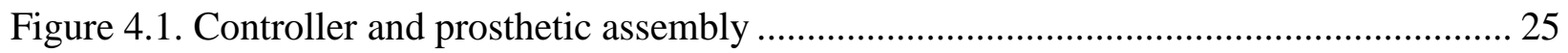

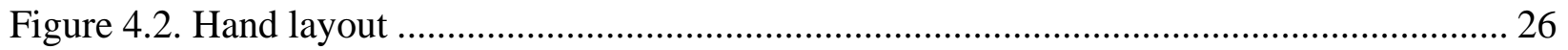

Figure 4.3. Sample schematic used to demonstrate torque calculations ................................... 27

Figure 4.4. Command and response signal recording process ............................................... 30

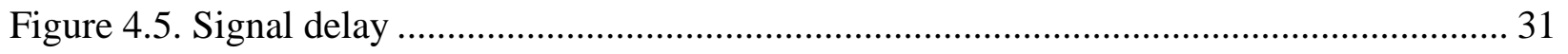

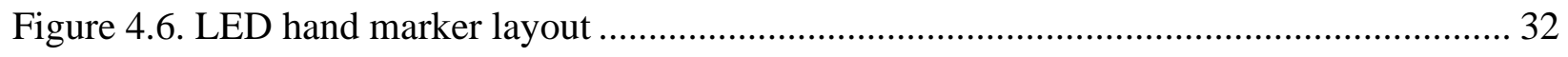

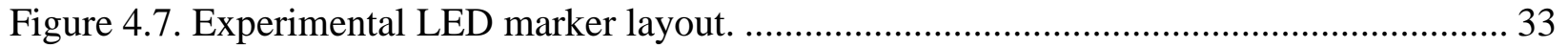

Figure 4.8. Local coordinate system setup..................................................................... 34

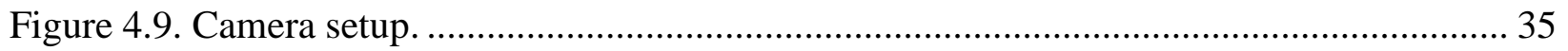

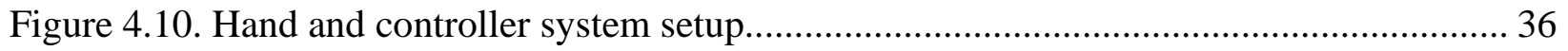

Figure 5.1. Examples of signals with representative delays ............................................... 42

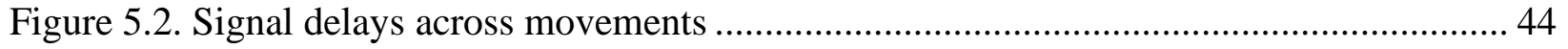

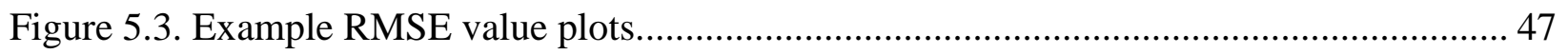

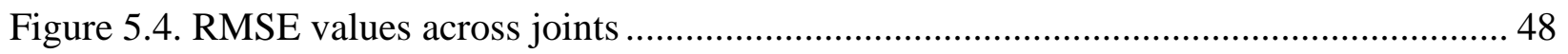




\section{List of Abbreviations}

\begin{tabular}{|c|c|}
\hline ADL & Activities of Daily Living \\
\hline CAD & Computer Aided Design \\
\hline CG & Center of Gravity \\
\hline CM & Center of Mass \\
\hline CMC & Carpometacarpal \\
\hline DIP & Distal Interphalangeal \\
\hline DOF & Degrees of Freedom \\
\hline EMG & Electromyography \\
\hline FDM & Fused Deposition Modeling \\
\hline MCP & Metacarpophalangeal \\
\hline NRMSE & Normalized Root Mean Square Error \\
\hline PETG & Polyethylene Terephthalate Glycol \\
\hline PIP & Proximal Interphalangeal \\
\hline PLA & Polylactic Acid \\
\hline RMSE & Root Mean Square Error \\
\hline ROM & Range of Motion \\
\hline SHAP & Southampton Hand Assessment Procedure \\
\hline TPU & Thermoplastic Polyurethane \\
\hline
\end{tabular}




\section{Chapter 1: Background and Literature Review}

\subsection{Overview}

Chapter 1 serves as an introduction to some of the major topics discussed throughout the thesis. This includes topics such as the upper limb anatomy, the relationship between mechanics and the human anatomy, a general prosthetic review, and thesis outline.

\subsection{Upper Limb Anatomy}

The upper limb refers to the combination of the upper arm, forearm, and hand, as shown in Figure 1.1 (Forro \& Lowe, 2019). Each segment is separated by joints whether it is the elbow joint between the upper arm and forearm or the wrist joint between the forearm and hand. Joints allow for the segments to move with respect to one another, providing functional advantages over situations in which joint movement is hindered. The focus of the following sub sections is to introduce anatomy specific to the hand and forearm as well as the wrist's structure and function.

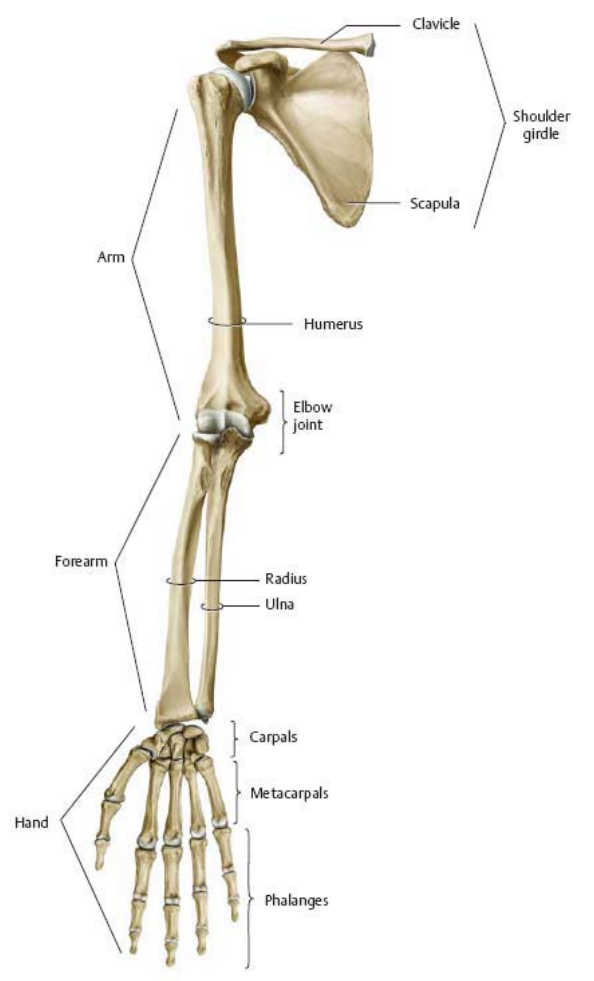


Figure 1.1. Upper limb anatomy. The main upper limb segments are shown. The elbow joint connects humerus to radius-ulna, and the wrist joint (not pictured) connects radius-ulna to hand segments (Arm and Shoulder Bones Anterior View and Posterior View, 2018).

\subsubsection{Hand and Forearm Anatomy}

The hand and forearm comprise 29 of the 30 bones in the upper limb. Describing from distal to proximal segments, fingers consist of the proximal, middle, and distal phalanx bones, but the thumb has only the distal and proximal phalanges (BCcampus, 2013a). Following the phalanges bones are the five metacarpal and eight carpal bones, shown in Figure 1.2. Next are the radius and ulnar bones connected via the radiocarpal wrist joint (Bair \& Gondal, 2019).

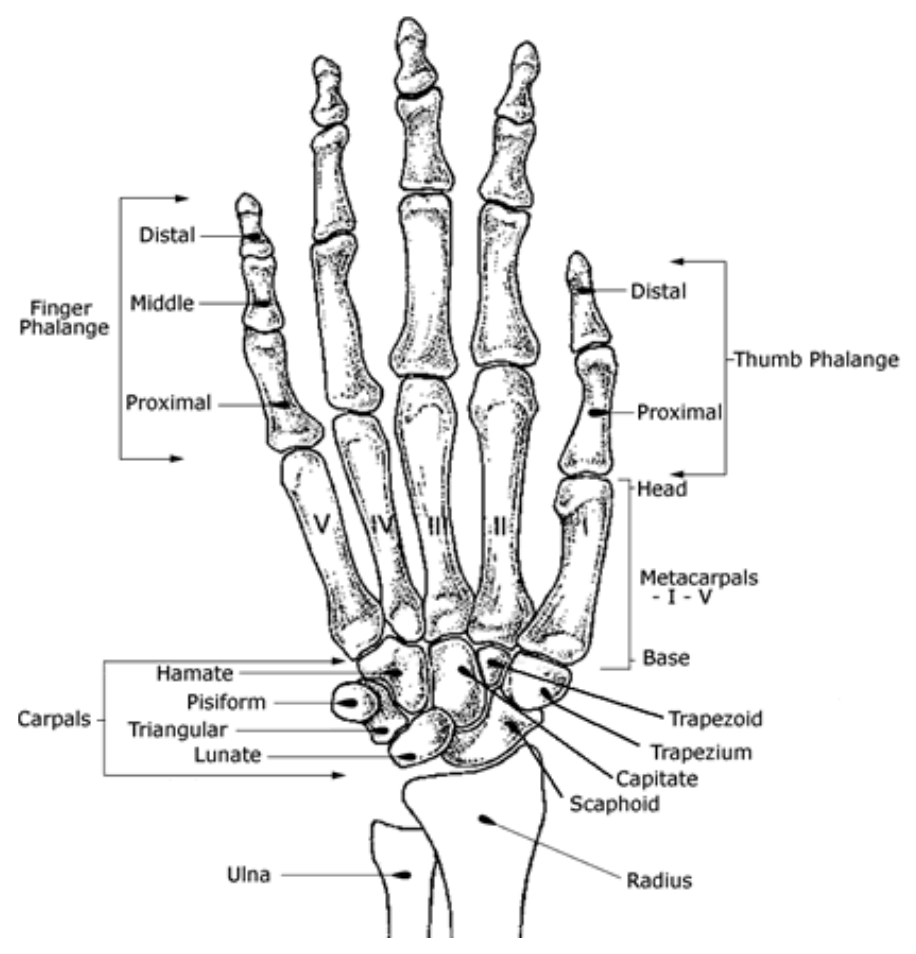

Figure 1.2. Anatomical layout of the left hand. The hand is described by the phalanges, carpals, radius, and ulna (Anatomy of the Bones: Hand). 


\subsubsection{Wrist Structure and Function}

There are three main planes that describe the body orientation: sagittal (left and right), frontal/coronal (front and back), and transverse (upper and lower) (Figure 1.3) (The Three Anatomical Planes of Movement, 2016). The wrist rotates in all three planes: flexion-extension occurs in the sagittal plane, radial-ulnar deviation occurs in the frontal plane, and pronationsupination occurs in the transverse plane (Crisco et al., 2011; BCcampus, 2013b). The wrist rotates because of the movement of the radius and ulna bones, and can be captured with the simplified radius-ulna models (Yough et al., 2021). This 3 degree of freedom (DOF) joint, along with the bones in the hand and forearm are essential activities of daily living (ADLs). ADLs are described as movements that can measure a person's functional ability to independently take care of theirself; however, for those who have suffered from transradial amputations, only three of the 30 bones remain (Edemekong et al., 2020). A transradial amputation is when the bones in the forearm become severed and only part of them remain (National Academies of Sciences, Engineering, and Medicine, 2017). In addition to the lack of bones, after an amputation, muscles have also been severed or completely lost during this procedure. One way to replace the function of the missing limb is to wear a prosthetic. 


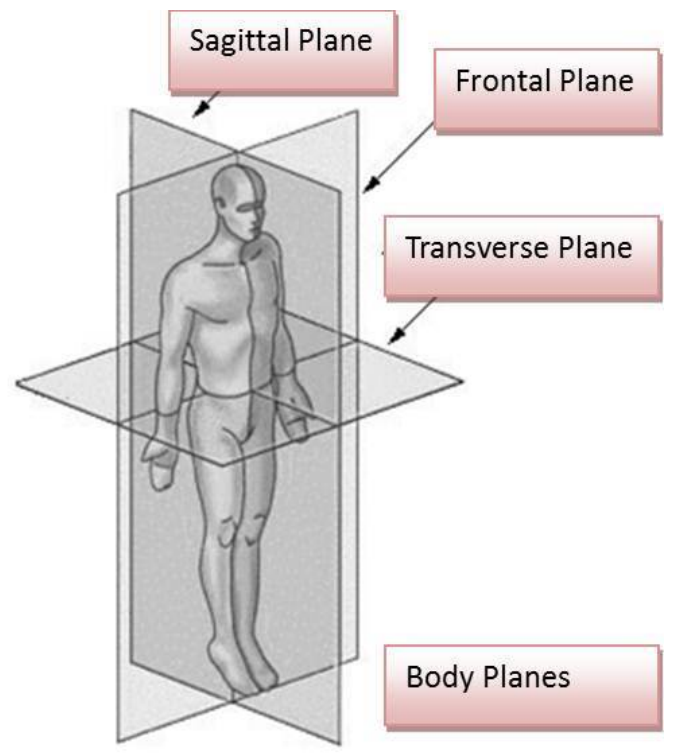

Figure 1.3. Anatomical planes. The body is described in three planes: sagittal, frontal, and transverse planes (The Three Anatomical Planes of Movement, 2016).

\subsection{Mechanical Relationship to Anatomical Structure}

The human arm is an over-actuated system where the number of muscles exceeds the number of joints. Even if this fully actuated system were to be replaced with a prosthetic, to independently actuate each DOF would be a difficult task due to weight and spatial restrictions (Semasinghe et al., 2019). Under-actuated systems can reduce space requirement in a mechanical design. In addition, the principle of remote actuation could be used through the implementation of a Bowden cable system. What was originally used for bicycle brakes, Bowden cables have been integrated into both body powered and myoelectric prosthetics (Bowden Cable, 2012). The principles of this cable system are similar to human mechanics where muscles are connected to the fingers by tendons to open and close the hand (Bordoni \& Varacallo, 2019). Instead of tendons sliding through a sheath, in a prosthetic the muscles are replaced by actuators and the tendon and tendon sheath are replaced by an inner cable and outer tube, respectively (Bordoni \& Varacallo, 2019). This mechanical setup can be seen in Figure 1.4. 

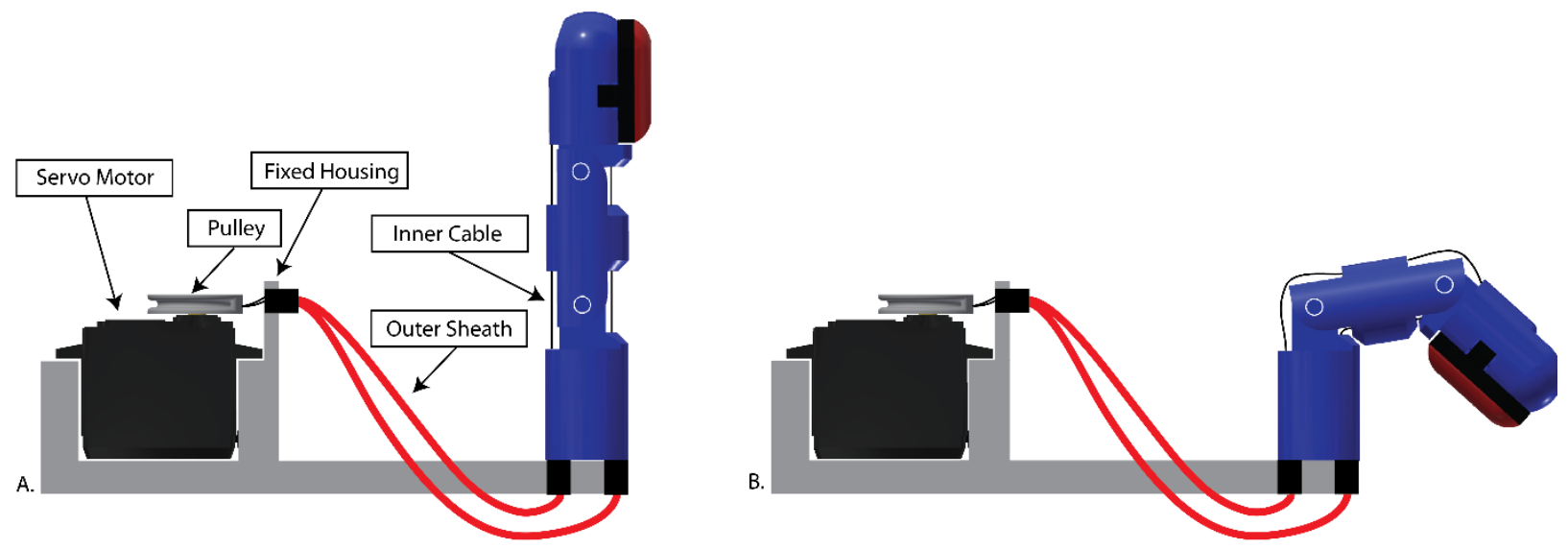

Figure 1.4. Bowden cable diagram. A. Demonstration of an extended finger with all main components of the Bowden cable system labeled. B. Demonstration of a flexed finger when the pulley is rotated (Ramirez et al., 2019b).

\subsection{Prosthetics Review and Manufacturing Overview}

Originally constructed from heavy materials such as iron and steel, modern prosthetics use lighter alternatives, compatible with 3D printing, including polycarbonates and carbon fiber (Timeline, 2015). 3D printing has become a more popular manufacturing method due to ability to produce lightweight parts, reduced time from conception to final production, and low overall cost. Several types of additive manufacturing methods exist including binder jetting, materials jetting, powder bed fusion, and materials extrusion (Gibson et al., 2015). Although materials extrusion is not quite as fast as material jetting or as precise as binder jetting or powder bed fusion, it produces some of the strongest parts available using additive manufacturing and can be done using several types of materials. In addition, materials extrusion, or more specifically fused deposition modeling (FDM) printers can be purchased starting at $\$ 200$ or less making this affordable. Figure 1.5 shows an example of FDM printing. Complex designs have been developed to include individual finger actuation with wrist pronation-supination and less frequently with wrist flexion-extension and 
radial-ulnar deviation. Although prosthetics have become more advanced, some prosthetics continue to be used only for cosmetic purposes.

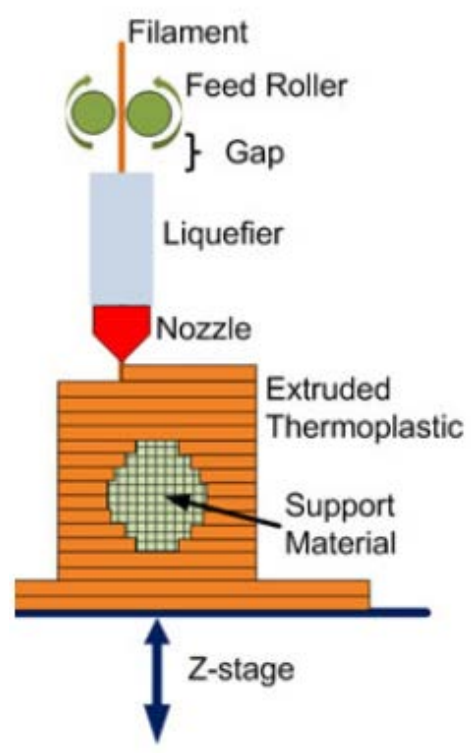

Figure 1.5. 3D printing process. The filament is fed into the liquefier and then the "liquid-like" material is extruded onto the build plate (Material Extrusion).

\subsubsection{Prosthetic Classifications and Control Techniques}

There are two main categories of upper limb prosthetics: active and passive (Ottobock, 2015). Passive devices serve as a cosmetic replacement to a missing limb generally restoring little to no function (Maat et al., 2018). Two subcategories fall under passive devices: prosthetic hands and prosthetic tools. One of the main differences between them is that prosthetic hands are meant to be used for several types of tasks whereas prosthetic tools are typically designed for a specific task Static and adjustable capabilities are listed under both categories and refer to either a stationary device or one that moves when it interacts with the healthy hand or the environment, respectively Unlike passive devices that lack independent control, active devices are capable of functioning independently. 
Active devices can serve as a cosmetic replacement while restoring function and include both body powered and electric powered devices (Ottobock, 2015). The body powered group includes cable and harness systems such as the Bowden cable system. Body powered prosthetics rely on the residual limb and the affected side of the body to generate enough force to control the prosthetic with movements such as opening and closing the hand (Weir, 2005a, p. 32.28). Externally powered prosthetics include switch, pneumatic, and myoelectric controls and do not require the residual limb to do any work to operate the device (Weir, 2005a, p. 32.2,32.26). The most common type of this category is myoelectric control and will be the topic of discussion. Myoelectric control is the use of electromyographic (EMG) signals, which are the electric potentials measured during muscle contraction, to carry out movement of the end effector (Reaz et al., 2006). There are many categories of myoelectric control including on/off, proportional, direct, pattern recognition, regression, posture, and finite state machine (Geethanjali, 2016). The on/off control strategy is the simplest since there are only two options for control. The proportional activation is complex, but it can take into account the dynamical properties of arm during movement and task dexterity. Opening and closing the hand as all or none can be intuitive with the binary control, but it lacks the nuanced aperture control needed in grabbing objects of different size and shape. The proportional control may be achieved through the direct scaling of EMG to the rate of opening closing of hand (Gaetani et al., 2019). The EMG signals could control different motions generated by the powered prosthetic.

Another type of prosthetic that would take features from both body powered and externally powered devices is a hybrid prosthetic. This could work by combining electrically powered servomotors with a Bowden cable system to remotely actuate the device. Although, there are several control strategies that can be implemented, the first step is choosing type of prosthetic. If 
an active prosthetic is chosen, then the next step is to weigh the advantages and disadvantages of the devices in this category.

On one hand, body powered devices are typically lighter weight, lower cost, and can lift heavier objects; however, they are not as aesthetically pleasing and generally do not have the dexterous capabilities required for ADLs (Uellendahl, 2017; Ottobock, 2015). On the other hand, myoelectric powered prosthetics tend to be heavier and more expensive, but are able to deliver the energy of missing muscles, have high DOF capabilities, require less compensation, and have more independent actions (Uellendahl, 2017; Ottobock, 2015; Pasquina et al., 2015). For example, all five digits could flex independently for an externally powered prosthetic; whereas, a body powered prosthetic could only open and close the hand as a single action. The expansion of movement repertoire, including independent finger control and wrist flexion-extension, is only one of many preferences that amputees have regarding prosthetics. Others include affordability, sensory feedback, dexterity, prosthetic durability, socket comfort, and weight. Failure to fulfill these preferences can lead to prosthetic abandonment (upwards of 20-30\%) (Benz et al., 2016; Cordella et al., 2016). We have no solution that captures all the desired attributes, and the selection of prosthetics remains a subjective task. Some people consider cost, and others focus on weight over function and cost, or aesthetics and individual finger control over weight (Benz et al., 2016; Biddiss et al., 2011; Cordella et al., 2016). Balancing complexity and function can be difficult. While some simple devices (few DOFs) can perform tasks well, others underperform and cannot complete tasks. Designs with increased complexity could be difficult to control and add more weight. This might deter people due to the higher learning curve. 


\subsubsection{The Balance Between Complexity and Functionality}

The human hand is a complex structure comprised of 27 DOFs (ElKoura \& Singh, 2003). Due to physical limitations, creating a wearable prosthetic capable of individually actuating each DOF is challenging; therefore, developing underactuated devices to control different DOFs deemed “important”, is the current approach. One way to determine which DOFs are necessary to actuate is by evaluating a device’s reach-to-grasp ability. Anthropomorphic reach-to-grasp is an essential function of hand prosthetics. The grasp itself can be further subdivided into two main types-power and precision grasps. Spherical grip, cylindrical grip, hook grip, and plate or lateral prehension are categorized as power grasps, and “chuck” or tripod position, lateral prehension, and pincer grasp are categorized as precision grasps (Mandich, 2015). Simple one DOF (hand open/close) devices can complete some power grasps such as the Unlimbited Arm v2.1 and Phoenix v2 Hand by Enabling the Future; however, this simple body-powered design sacrifices function at the cost of a low complexity design (“Which Design?,” 2016). Myoelectric devices with multiple DOFs, such as the Luke Arm, Michelangelo Hand, Bebionic3 Hand, iLimb Quantum, and TASKA, can perform some of the most common grasps for ADLs including power, precision, and lateral pinch grasps. Most of the devices have wrist pronation-supination and some have individual finger control (either all fingers or the index and thumb), but there are less prosthetics available commercially or developed in research with flexion-extension capabilities. A detailed table of each commercial and research-based devices can be seen in Appendix A.

\subsection{Thesis Overview}

The objectives were to simulate, design, manufacture, and test a newly developed 6 DOF prosthetic. This process was broken into several chapters. Chapter 2 serves an introduction focused on presenting the research problem. In this chapter I discuss where current research stands on 
prosthetics and the wrist flexion-extension DOF, and where there is a gap. This is followed by the solution approach where I present the steps taken to bridge this gap. The next chapter, Chapter 3, revolves around the simulation of the original robotic arm design. Included is detailed information on the modeling methods using the database of movement tasks generated in our previous work (Sobinov, 2019). Chapter 4 describes the device design, its development with hardware selection, and the manufacturing method. I explain in detail how the control system worked along with the process for synchronizing the command and response signals. Furthermore, a step-by-step solution is shown for calculating the joint angles starting with the marker placement. Device testing is also explained including equipment setup and the experimental tasks that were performed. The results are presented in Chapter 5. These include simulations used to perform the hardware selection and experimental testing. I performed calculations of the latency between the command and response signals and the root mean square error (RMSE) between the kinematics of reference and response angles. The results are discussed and summarized in Chapter 6. This section details other design choices that were considered, but not selected, as well as possible improvements that could be made. Discussion of the data is presented for both the simulation and experimental results as well as overall experimental limitations and mitigations. Finally, future work is discussed, followed by a wrap up of the project in the concluding section. 


\section{Chapter 2: Introduction}

\subsection{Overview}

This chapter provides a brief introduction to the problem including current prosthetic research and the gap between what is necessary for a functional prosthetic, and why it is not being delivered. A solution approach is presented focused on filling this gap. It includes the design, manufacturing, and testing of an alternative multi-DOF device.

\subsection{Problem}

The wrist joint is used in at least 24 different ADLs, but when the wrist is unable to function at full capacity postural actions at other joints may be required (Neumann, 2017). For example, shoulder compensation increases when the wrist is splinted during reaching over barriers (Mell et al., 2005). Even rudimentary wrist flexion-extension function (limited to three positions: extension, neutral, and flexion) has functional advantages over the non-flexible wrist in every grasp during the Southampton Hand Assessment Protocol test (Kyberd, 2012). Despite the proven benefits, wrist flexion-extension is less frequently seen among commercial and research prosthetic devices (Appendix A). One issue with this is that it is not always clear that increased effectiveness and improved function are associated with the addition of wrist flexion-extension (Deijs et al., 2016; Olsen et al., 2019). In Deijs et al., the addition of wrist flexion-extension did not result in functional improvements; however, there was high inter-individual variability (15\%-50\%), indicating that there may have been confounding factors shaping performance (Deijs et al., 2016). Although no compensatory change in shoulder posture was detected between the wrist conditions, the compensation associated with trunk motion was not measured, and it was shown in a related study (Bertels et al., 2009). Similarly, wrist flexion-extension function did not result in faster task completion in another study where neither shoulder nor trunk movements were measured (Olsen 
et al., 2019). Thus, the lack of improvements in this type of study could have been due to the reliance on the compensatory mechanisms at other body segments. When the compensation was matched between conditions, the addition of wrist flexion-extension resulted in the improved performance. Thus, it is imperative to measure compensatory movements and include them in the consideration of performance. Regarding the speed of task completion, speed could also be affected by lack of proper compensatory measurements as relying on other parts of the body to complete the task would skew the results. We then might ask the question, if wrist flexionextension is beneficial, why is it not included in commercialized prosthetics? To answer we need to examine the challenges of using wrist flexion-extension in addition to the pronation-supination wrist action. The limitations include prosthetic weight, size, and overall controllability. It has been suggested that pronation-supination and not flexion-extension is more important for the wrist function; therefore, when designing a simple device, an added pronation-supination DOF is preferred over the flexion-extension DOF despite the benefits of reduced compensation (Fan et al., 2016; Kanitz et al., 2018; Kyberd et al., 2011; Olsen et al., 2019). The average hand weighs around $0.4 \mathrm{~kg}$, and, subsequently, hand prosthetic weight is recommended to be under $0.5 \mathrm{~kg}$, which limits the number of independent actuators and their power usually correlated with weight (Belter \& Dollar, 2011). Device size is typically defined by anthropomorphic measurements for functional and aesthetic reasons. The space limitation affects may be further exacerbated by the space required for the interface with the residual limb left, the less space there is to work with inside of the device. Finally, controlling two DOFs at the wrist is difficult. Not only must both be able to move independently, but they must also move together in certain circumstances. For example, the simultaneous control of both pronation-supination and flexion-extension during complex tasks, like picking up a teacup, is more intuitive than the control of single actions. This is not easily 
achievable by simply hooking up a motor directly to the hand as is seen with pronation-supination. Despite the potential drawbacks of adding wrist flexion-extension, this capability may improve functional performance.

\subsection{Solution Approach}

The goal of this study was to add wrist flexion-extension to an open-source 3D printable robotic arm. The starting point in our development was a 7-DOF robotic arm (i.e., InMoov) with the following DOFs: wrist pronation-supination, thumb abduction-adduction, and thumb, index, middle, ring, and small finger flexion-extension (Hand \& Forearm). This device was chosen for its open-source control and simple design. Humanoid anthropomorphic naturally dexterous intelligent hand is an example of a device developed from the same open-source foundation, which was modified to include features such as force sensors and position sensors for improved prosthetic control (Brenneis et al., 2017). Despite both the original and modified devices having similar DOFs, neither included the wrist flexion-extension DOF. The new device was designed and demonstrated to move each available DOF and simultaneously multiple DOFs in a diverse set of realistic hand movements. The purpose of testing individual finger movements was to demonstrate movement over the device's ROM. The purpose of testing full hand movements was to demonstrate prosthetic performance in movements similar to those commonly seen during ADLs. These goals were accomplished in three steps. First, the prosthetic design was instructed by simulations of the robotic arm with an added wrist flexion-extension DOF to provide suggested servomotor requirements. Second, I developed a prototype using computer aided design (CAD) and manufactured it using 3D printed rapid prototyping. Finally, I tested it by demonstrating its movement range and dynamics. 


\section{Chapter 3: Simulation}

\subsection{Overview}

This chapter details the design of a new 6-DOF device presented in the study. Initially introduced are the preliminary simulations and their involvement in hardware selection. Following this are the design and manufacturing, control methods, performance metrics, and the experimental setup and testing.

\subsection{Modeling}

In this study, I modified the original robotic arm to include wrist flexion-extension, and used Simscape Multibody (MATLAB) to perform static and inverse dynamic simulations. The purpose of these simulations was to obtain the torques generated at the wrist during flexion-extension and pronation-supination for various arm postures, spherical weights, and wrist rotational speeds. The 20-DOF model consisted of 20 major segments with inertia defined by segment geometry (from the CAD model) and uniform material density. To simplify the simulation, only one DOF could move at a time: either wrist flexion-extension or pronation-supination. Throughout the simulation, the fingers remained flexed around a spherical solid: this was representative of moving a physical object encountered in daily life. This setup can be seen in Figure 3.1 (A-C) with the sphere placed at the palm's center of mass (CM), to simplify analytical calculations. Within the global coordinate system, the model was oriented with the positive $x$-axis pointing from lateral to medial, the $y$-axis

pointing from the posterior palm to the anterior palm, and the z-axis pointing from proximal to distal along the arm segment, also shown in Figure 3.1 (A-C). Local coordinate systems were also established at each segment. 

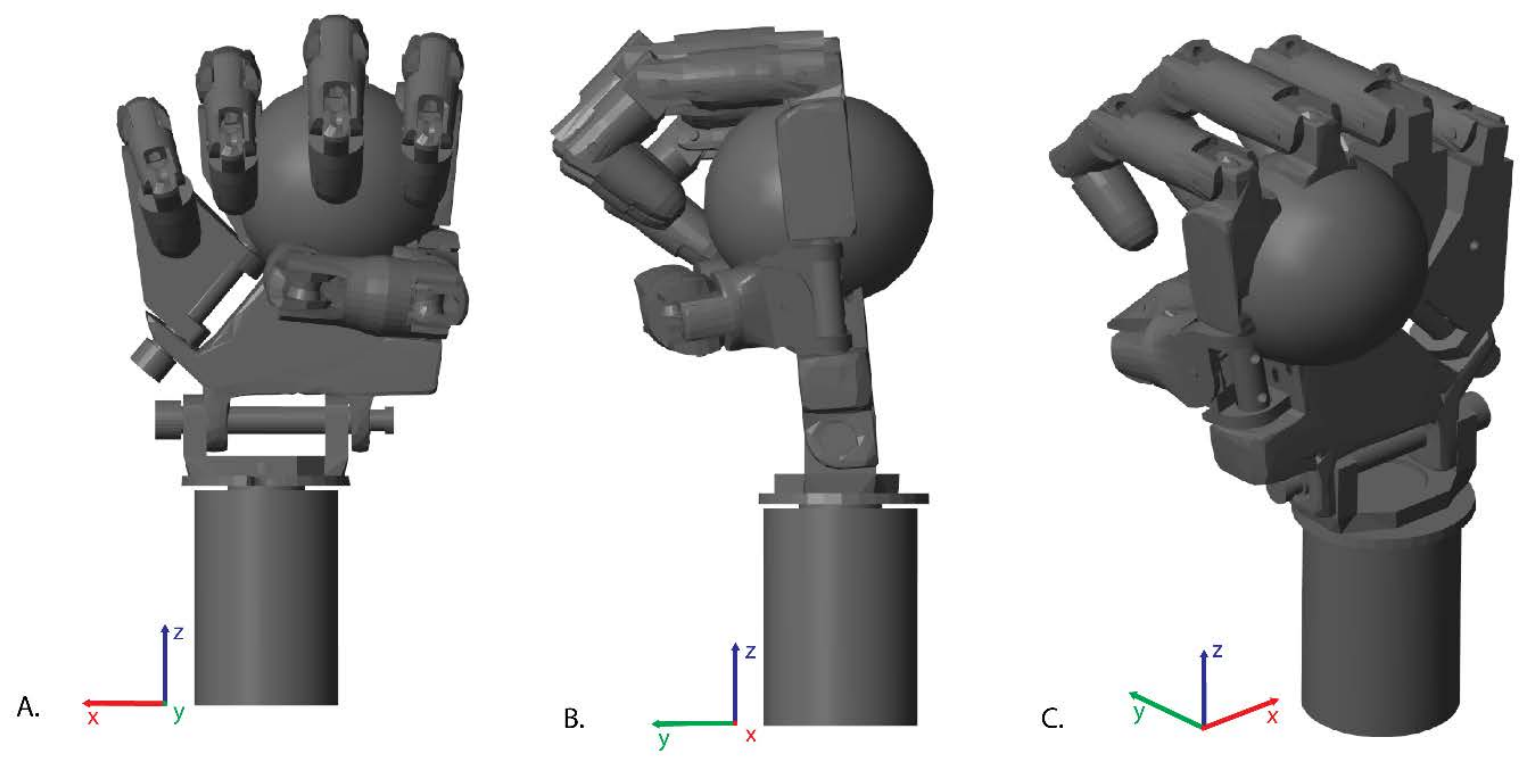

Figure 3.1. Neutral wrist orientation. A. Anterior, B. side, and C. isometric views of the model orientation within the Simulink environment.

The model was simulated using ode45 (variable step Runge-Kutta), with a relative tolerance of 10 ${ }^{3}$. Since the model was not run in real-time, the variable step size was not an issue. The accuracy of the model was determined by comparing two sets of simulations with a maximum step size set to either $10^{-3} \mathrm{~s}$ or $10^{-5} \mathrm{~s}$ (Important Concepts and Choices in Physical Simulation - MATLAB \& Simulink). Performing simulations at both step sizes allowed for the ability to verify the numerical stability of the solution. This was tested by taking the difference in the step sizes, and if there was no difference then the solution was stable and it was acceptable to use either the larger step size of $10^{-3} \mathrm{~s}$ or the smaller value of $10^{-5} \mathrm{~s}$.

\subsection{Movement Tasks}

Two movement types were discussed in this section. The first was used to help determine hardware requirements, one of the preliminary steps in the design process. The second was used during experimental testing to demonstrate kinematic movements of an ideal human hand. 


\subsection{Hardware Requirements}

Simulations were performed to determine the minimal configuration of the required servomotor hardware. I defined the requirements with various combinations of postures (horizontal, diagonal, and vertical limb orientation), rotational speeds (15, 30, 60, $120 \mathrm{deg} / \mathrm{s})$, and weights (0, 1, 2, $6 \mathrm{~kg})$. In the dynamic flexion-extension movement, the DOF was placed in the initial neutral position (arm oriented with fingers parallel to the forearm) and moved into full flexion, returned to neutral, then full extension, and finally returned to the neutral position. Similarly, for the pronationsupination DOF, the wrist was moved through the sequence: neutral, full pronation, neutral, full supination, neutral.

To simulate the effects of gravity, the model remained fixed in the global coordinate system (CS) and the gravity vector was rotated to simulate the different postures. For example, in the case of Figure 3.2 (A) the arm is in a horizontal position because gravity is acting in the $\mathrm{x}$ direction (with a $[9.81,0,0] \mathrm{XYZ}$ vector). Similarly, in the diagonal and vertical orientations, gravity points in the $-\mathrm{XZ}$ (gravity is partially in both the $\mathrm{x}$ and $\mathrm{z}$ directions) and $\mathrm{-Z}$ directions with vectors [6.936, $0,-6.936]$ and [0, 0, -9.81], respectively. 


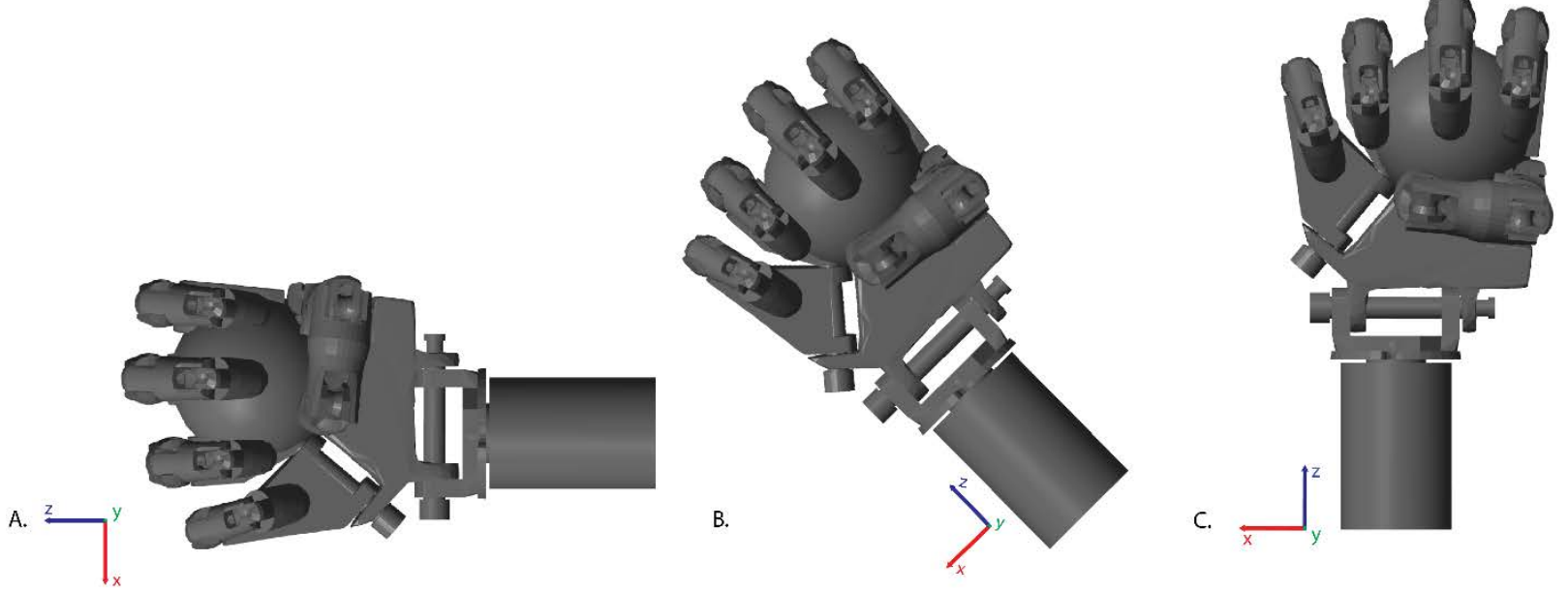

Figure 3.2. Arm postures based on the effects of gravity. A. Horizontal, B. diagonal, and C. vertical model orientations within the Simulink environment. These orientations represent the different positions of an arm during activities of daily living.

\subsection{Kinematics of an Ideal Human Hand}

Complex movements comprising ADLs served as the basis for selecting the repertoire of the simulated movements (Sobinov, 2019). Individual DOF movement profiles were created to simplify complex motions and better display the capabilities of each DOF separately. The input trajectories were created using cubic splines to map the range of motion (ROM) of the DOF. Originally the data included the position, velocity, and acceleration for each joint of an ideal human hand, but it was modified to only include positional changes. The ideal data is defined by the ROM of an average human hand. Using a set period of time this the data is mapped using a spline, between the ROM limits. This ideal database served as a metric of comparison between the accuracy of a human hand and that of the new 6-DOF device.

\subsection{Analytical Calculations}

The simulation, described above, was verified using analytical calculations. A simplified model was created, and the hand was represented by a simple rectangular shape. The weight was 
represented by a spherical solid. One assumption was that calculating the moment of inertia for the hand separately from the sphere would not cause significantly different results from the simulated ones. So instead, the weight of the hand $(\sim 0.2 \mathrm{~kg})$ was added to the sphere. Detailed equations are presented in Appendix B explaining how the forces, moments of inertia, and torques were calculated. The following briefly explains the three movement tasks that were verified and where the angles were measured to calculate the distance for the torque calculations.

The first movement was performed with the arm in a vertical posture. During this movement, the wrist was flexing and extending at a maximum rotational speed of $120 \mathrm{deg} / \mathrm{s}$ holding a $6 \mathrm{~kg}$ mass. The weight was represented by the red vector which always acts in the negative global $\mathrm{z}$ direction, as shown in Figure 3.3. This figure was adapted to be able to calculate the wrist torques in a horizontal posture as well. The second movement also examined the wrist during flexion-extension motion, but this the maximum rotational speed was $30 \mathrm{deg} / \mathrm{s}$ holding a $1 \mathrm{~kg}$ mass. In this movement the forearm was rotated $90^{\circ}$ clockwise such that the global $x$-axis was along the center axis of the forearm and the z-axis was perpendicular to the palm. Again, the weight vector acted in the negative z-direction. 


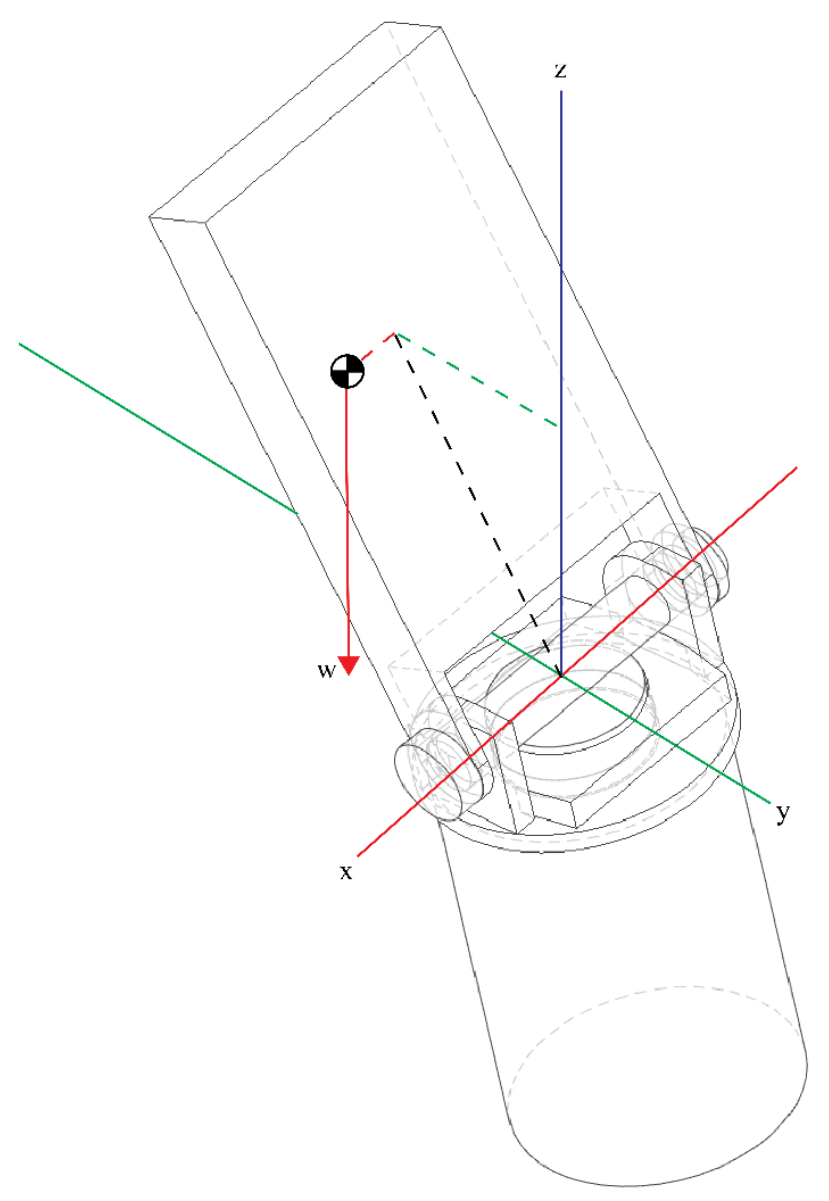

Figure 3.3. Diagram for analytical wrist flexion-extension calculations. This diagram was used in the calculation of wrist torques, in a vertical posture, during flexion-extension. The figure was adapted to be used when the wrist was horizontally positioned.

The third movement was also in the horizontal direction. This time wrist pronation-supination was the prescribed motion moving at a maximum rotational speed of $60 \mathrm{deg} / \mathrm{s}$ holding a $6 \mathrm{~kg}$ mass. Figure 3.4 shows several examples of the wrist during pronation from which the angles were calculated. These angles were used in conjunction with the distances (represented by green or red lines to the CG) and the weight to calculate the toque about each axis. Figure 3.4 (A) shows the wrist in a neutral posture with the CG offset by an angle $\psi$ from the $\mathrm{x}$ axis. This angle remained constant through the entirety of the movement. Panel B represents any angle, $\theta$ between $0^{\circ}$ and 
$90^{\circ}$. The sum of $\theta$ and $\psi$ represents the angle of the CG measured from the global $\mathrm{x}$ axis $\varphi$. Panel $\mathrm{C}$ represents when $\theta$ is at $90^{\circ}$. At this point the local $\mathrm{x}$ and global $y$-axis have aligned. The distance from the CG to the axes can be calculated with the two angles shown. Finally, panel D shows where the CG would lie at angles larger than $90^{\circ}$ but smaller than $270^{\circ}$.

A.

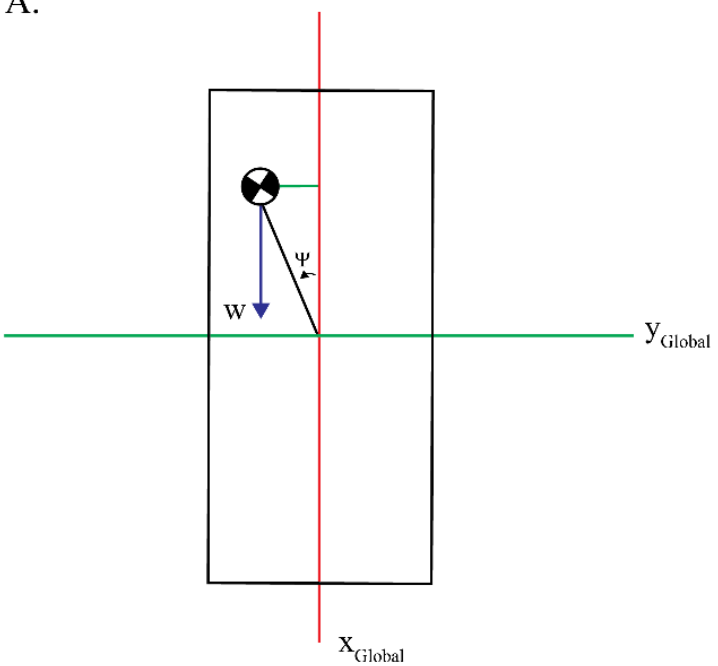

C.

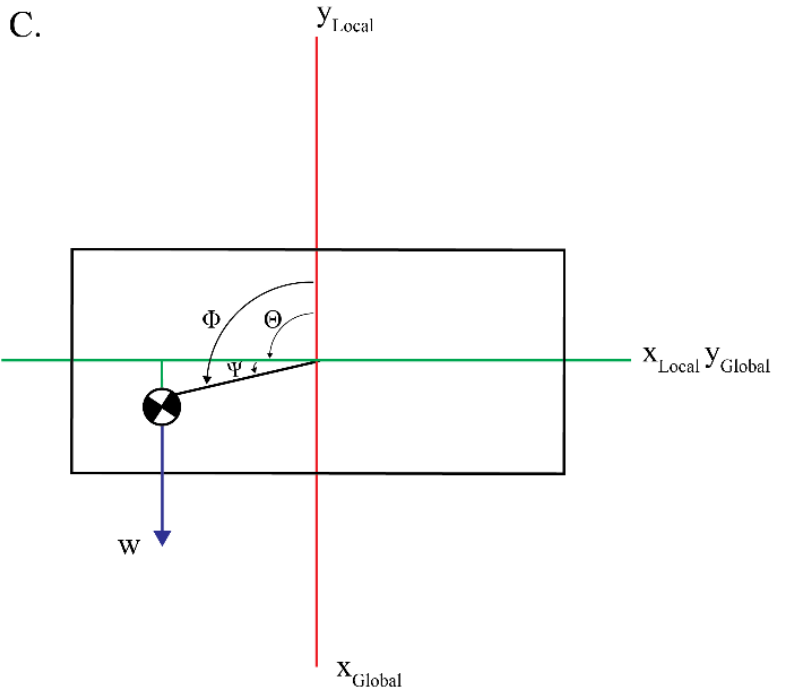

B.

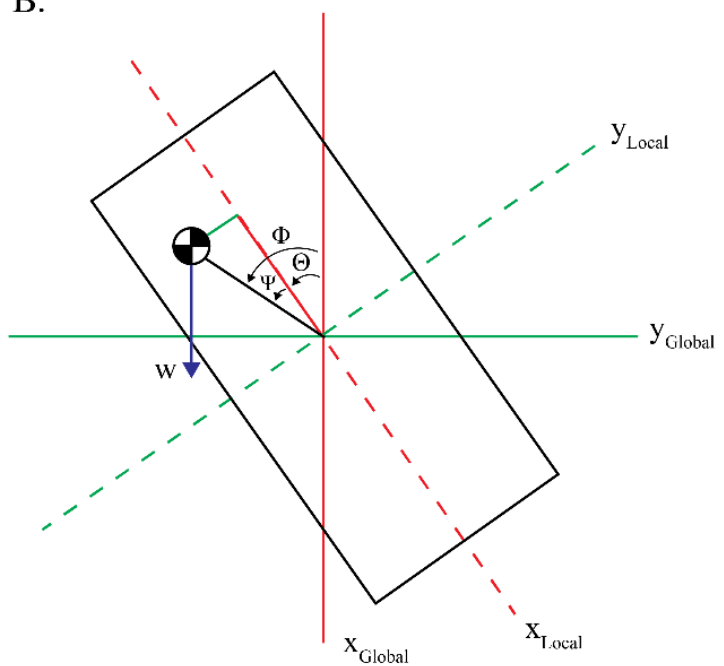

D.

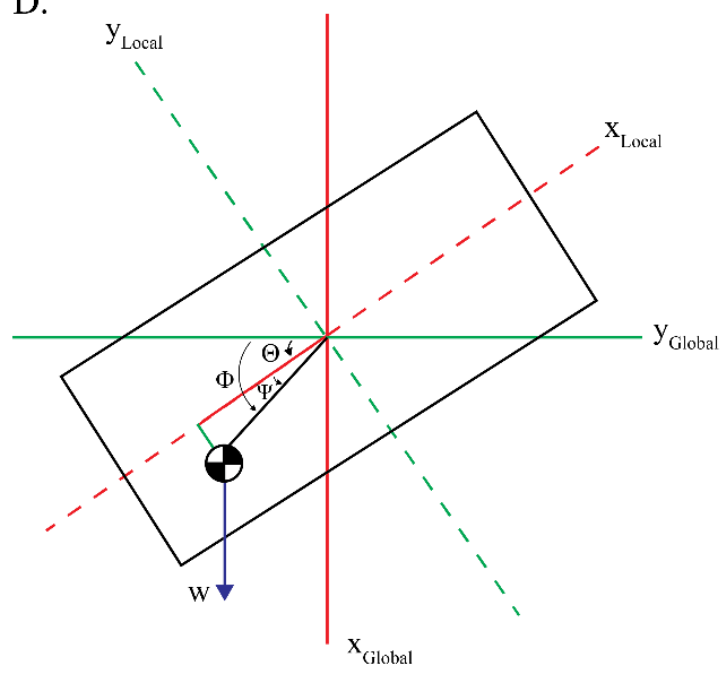


Figure 3.4. Diagram for analytical wrist pronation calculations. A. The wrist oriented in a neutral position $\left(0^{\circ}\right)$. B. Cases when the wrist has been pronated more than $0^{\circ}$ but less than 90 . C. The wrist has pronated to $90^{\circ}$ and the local $\mathrm{x}$ axis has aligned with the global y axis. D. This is when the wrist has pronated beyond an absolute angle of $90^{\circ}$ but less than $270^{\circ}$.

The other half of the pronation-supination movement was when the wrist was supinated. Figure 3.5 demonstrates one case of supination. It is similar to the pronation calculations except this time the sign has changed.

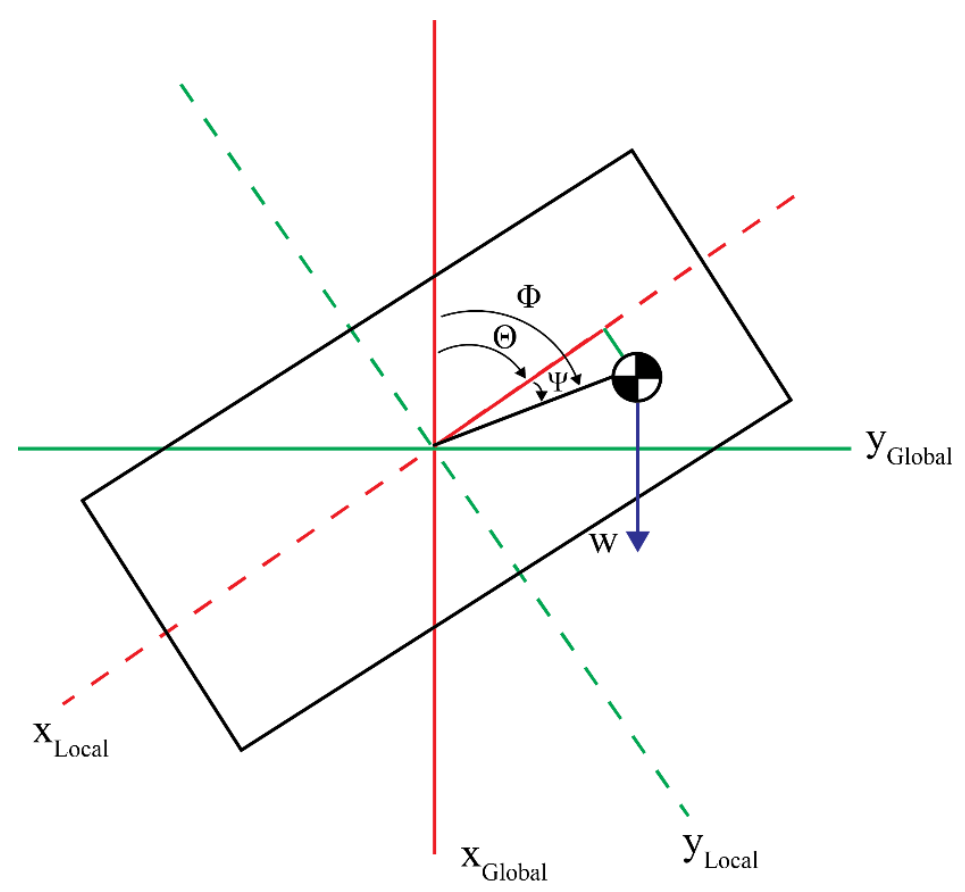

Figure 3.5. Diagram for analytical wrist supination calculations. This figure shows one position in which the distances were able to be calculated using the known angles above.

The equations of motion below were used to calculate the torques generated at the pronationsupination and flexion-extension axes. Calculating the sum of forces was the first step to determine the analytical torque, and this process can be seen in Appendix B. After the forces were summed 
in the $\mathrm{x}, \mathrm{y}$, and $\mathrm{z}$ directions, the next step was to determine the summation of moments about each axis (Hibbeler, 2013). This is shown in eq. (3-1) through eq. (3-3).

$$
\begin{aligned}
\sum M_{x}= & I_{x x} \dot{\omega}_{x}-\left(I_{y y}-I_{z z}\right) \omega_{y} \omega_{z}-I_{x y}\left(\dot{\omega}_{y}-\omega_{z} \omega_{x}\right) \\
& -I_{y z}\left(\omega_{y}^{2}-\omega_{z}^{2}\right)-I_{z x}\left(\dot{\omega}_{z}+\omega_{x} \omega_{y}\right) \\
\sum M_{x}= & I_{x x} \dot{\omega}_{x}-\left(I_{y y}-I_{z z}\right) \omega_{y} \omega_{z}-I_{x y}\left(\dot{\omega}_{y}-\omega_{z} \omega_{x}\right) \\
& -I_{y z}\left(\omega_{y}^{2}-\omega_{z}^{2}\right)-I_{z x}\left(\dot{\omega}_{z}+\omega_{x} \omega_{y}\right) \\
\sum M_{z}= & I_{z z} \dot{\omega}_{z}-\left(I_{x x}-I_{y y}\right) \omega_{x} \omega_{y}-I_{z x}\left(\dot{\omega}_{x}-\omega_{y} \omega_{z}\right) \\
& -I_{x y}\left(\omega_{x}^{2}-\omega_{y}^{2}\right)-I_{y z}\left(\dot{\omega}_{y}+\omega_{z} \omega_{x}\right)
\end{aligned}
$$

The I terms correspond to the mass moment of inertia with units of $\left(\mathrm{kg} \cdot \mathrm{m}^{2}\right)$, the $\dot{\omega}$ term corresponds to the angular acceleration ( $\left.\mathrm{rad} / \mathrm{s}^{2}\right)$, and the $\omega$ term corresponds to the angular velocity ( $\mathrm{rad} / \mathrm{s}$ ) all of which are with respect to axis of the attached subscript. The moment eq. (3-1) through eq. (3-3) are measured in units of Nm. Figure 3.3-Figure 3.5 were crucial to properly determine the mass moments of inertia as well as with properly determining the force and its direction in the summation of torques equations. Using eq. (3-4) through eq. (3-6), the torque about the $x$-axis and z-axes which correspond to the wrist flexion-extension and pronation-supination axes, respectively, was calculated (Hibbeler, 2013).

$$
\begin{aligned}
& \sum T_{x}=M_{x}+z F_{y}+y F_{z} \\
& \sum T_{y}=M_{y}+x F_{z}+z F_{x} \\
& \sum T_{z}=M_{z}+x F_{y}+y F_{x}
\end{aligned}
$$


The $M_{x}, M_{y}$, and $M_{z}$ values correspond to the moment outputs. The $x, y$, and $z$ values are the perpendicular distance to the forces in the $F_{x}, F_{y}$, and $F_{z}$ directions measured to the appropriate axis of rotation. 


\section{Chapter 4: Design and Testing}

\subsection{Overview}

The design for the new 6-DOF device was organized into three main sections: hand and finger, wrist and forearm, and the compact controller design. The section on the hand details the finger layout and mechanical design. The wrist and forearm section examines the motor placement, and the compact controller design discusses the components housed in the system as well as the Bowden cable system.

\subsection{Hand Design}

Weight and space limitations are two major constraints when designing a prosthetic. Weight reduction is necessary to minimize strain on the residual limb, and minimizing space is necessary such that there is room for the residual limb. Typically, prosthetics strive to be under $0.5 \mathrm{~kg}$; however, some research-based prosthetics can weigh as much as $2.2 \mathrm{~kg}$. To help increase space and reduce weight, I took advantage of the natural interdependency of the middle, ring, and small fingers with one another, and designed the hand to incorporate this ability. Using a Whipple-treelike mechanism, these fingers were coupled to minimize both space and hardware (as shown in Figure 4.1(B) and (C)). Unlike a typical Whipple-tree, the mechanism does not rotate; however, the design principle of a main cable actuating multiple cables was used (Cuellar et al., 2018; Meetze, 2020). The thumb and the index finger were the only two independent digits. Due to their independency, both digits flex-extend (or abduct-adduct) with their own pulley cable; however, the middle, ring, and small finger were coupled. 


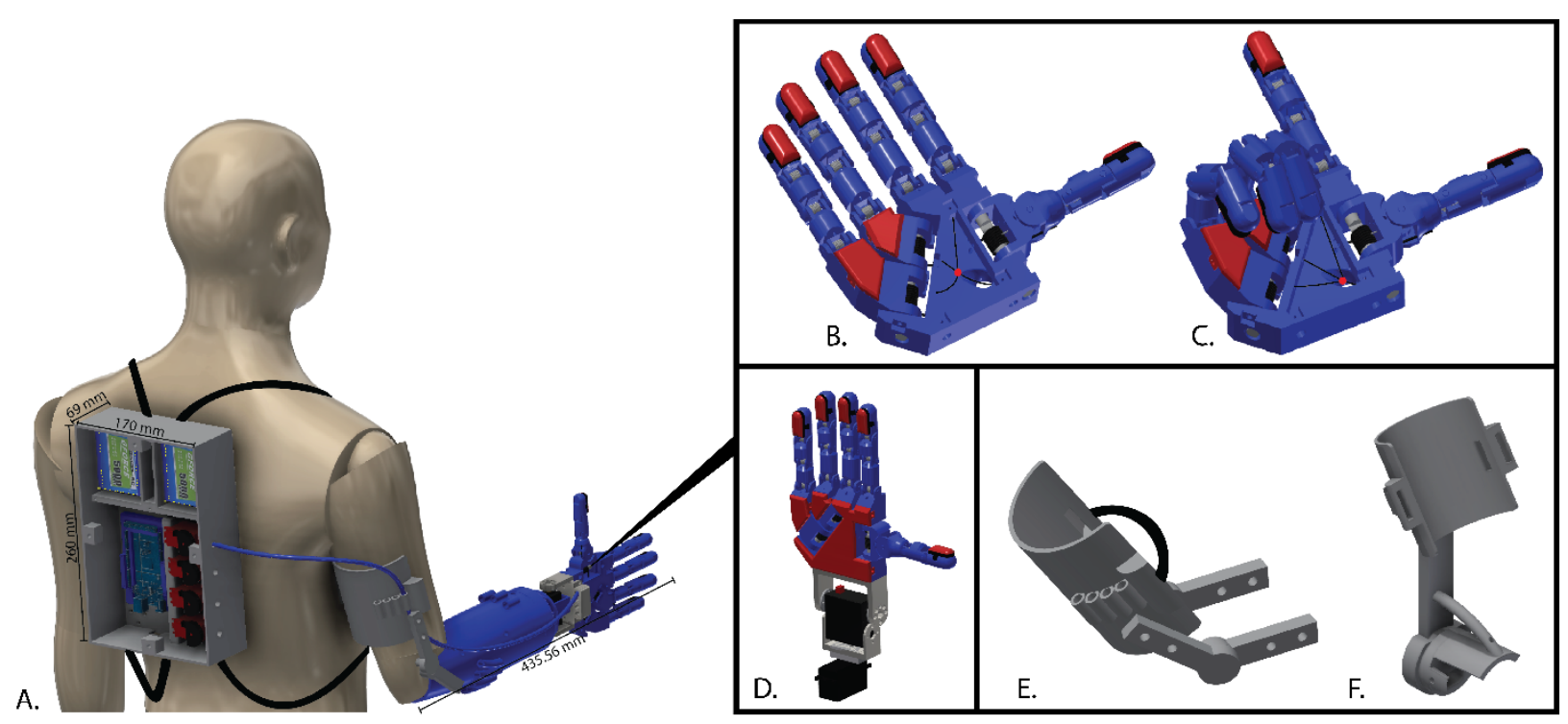

Figure 4.1. Controller and prosthetic assembly. A. Bowden cable system with controller and terminal device, B. the hand in an opened position with the "Whipple-tree" like mechanism, $\mathbf{C}$. demonstration of how pulling on the cable will close the middle, ring, and small fingers, D. the wrist design for flexion-extension and pronation-supination, E. bicep attachment for amputees, and $\mathbf{F}$. bicep attachment for healthy subjects.

The hand was designed (Autodesk Inventor, 2019) with four fingers and one thumb, to resemble the physical appearance of a human hand. In total, there were four actuated DOFs: thumb abduction-adduction, thumb flexion-extension, index flexion-extension, and the coupled flexionextension of the middle, ring, and small fingers. The layout of each finger was comprised of segments connected by joints. Each joint included a torsional spring held in place by a pin (as shown in Figure 4.2). This uses a similar approach to Dalley et al. (2009) by taking advantages of the natural extension properties of a spring (Dalley et al., 2009). A combination of springs and cables, instead of muscles and tendons, were used to flex-extend the fingers. The cable forced the fingers to flex when the servomotor was activated, while the springs passively actuated the system helping to stabilize and restore the hand to an extended position. 


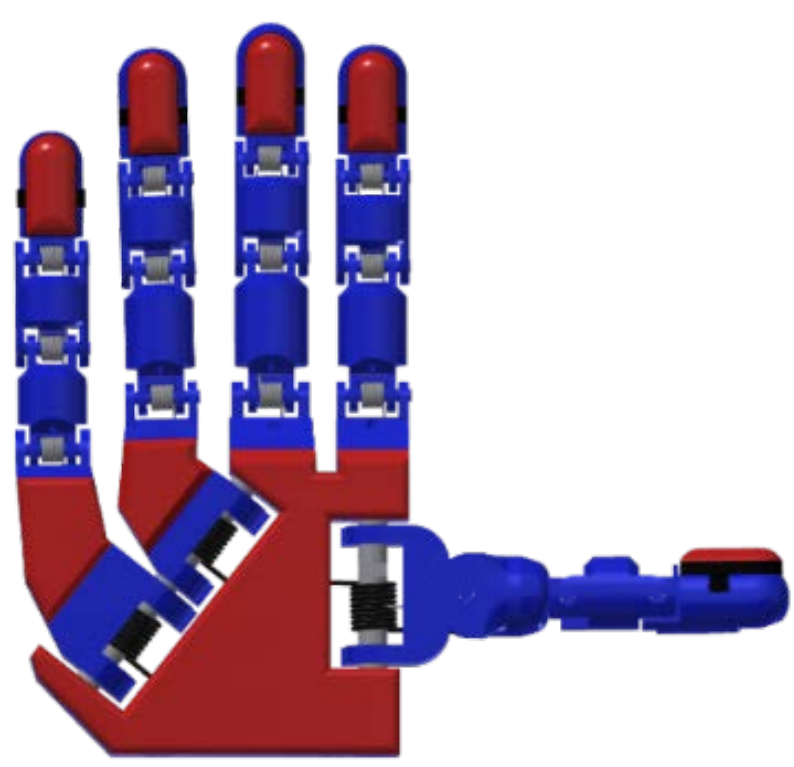

Figure 4.2. Hand layout. The hand diagram includes the palm as well as the thumb, index, middle, ring, and small fingers (right to left). Between each finger segment is a torsional spring held in place by a pin.

This system is known as forced-close: a force, in this case provided through a pulley system, caused the hand to close and when it was removed, the hand voluntarily returned to an extended position. The necessary spring torque required to restore the hand to an extended position was calculated to be approximately $0.014 \mathrm{Nm}$ and under for all joints. The estimated torque, $\tau$, was calculated as shown in eq. (4-1), with the spring represented by the orange circle to the bottom of Figure 4.3 (A, C) and left of Figure 4.3 (B) (block 1).

$$
\tau=\sum m_{1} d_{1}+m_{2} d_{2}+m_{3} d_{3}
$$

The mass for each block is represented by $m$ and the distance from the block's center of mass to the fixed point is represented by $d$. After performing the calculations, and testing multiple sets of springs, the springs that were selected for the project were a $180^{\circ}$ right hand wound spring with a maximum torque of $0.302 \mathrm{Nm}$ and a $180^{\circ}$ left hand wound springs with a torque of $0.0454 \mathrm{Nm}$. 
The springs with the larger torque were used at the joints where the finger segments attached to the palm, and the springs with the smaller torque were used in the remainder of the finger joints.

A.

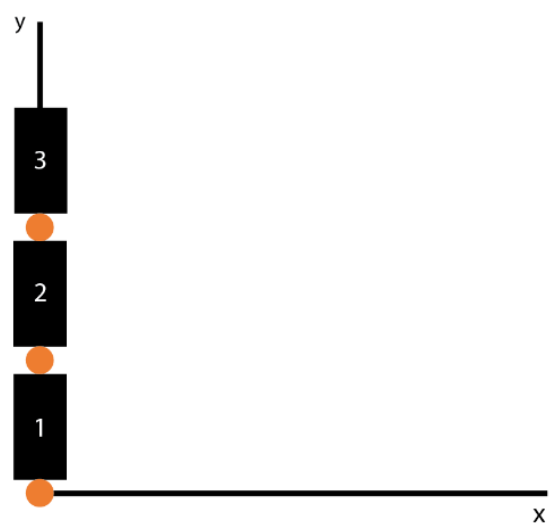

B.

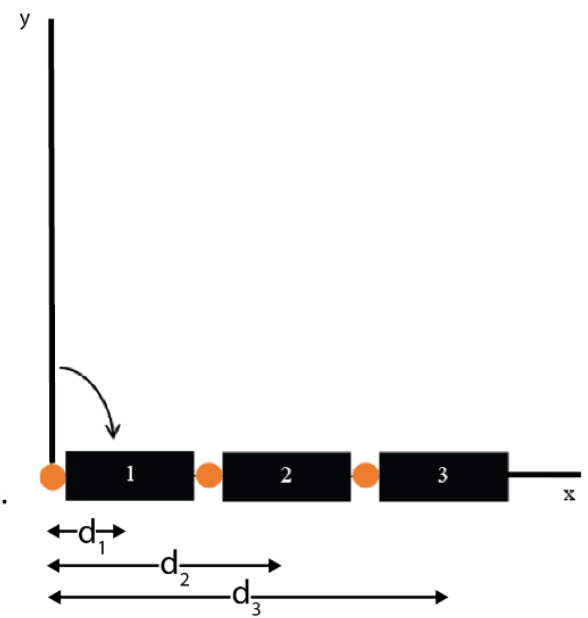

C.

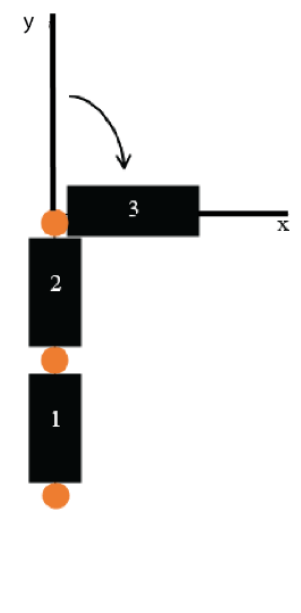

Figure 4.3. Sample schematic used to demonstrate torque calculations. A. Representation of the finger in an extended position with the springs in a neutral position. B. Representation of how the torque was calculated at the metacarpal joint (orange circle to the far left of block 1). $\mathbf{C}$. Representation of how the torque was calculated at a single segment.

\subsection{Wrist and Forearm Design}

The wrist was designed with the goal of increasing the actuated DOFs to incorporate not only pronation-supination, but also flexion-extension. A similar attempt to add these DOFs was seen in Olsen et al. (2019) (Olsen et al., 2019). I similarly used two servo motors in the wrist; however, the motors were directly attached with a bracket in between a low-profile servomotor for pronation-supination and a standard servomotor for flexion-extension. Figure 4.1 (D) shows how the servos were attached to one another. The servo for pronation-supination attached directly to the forearm. The forearm was a hollow segment that could be used for the interface with an amputee's residual limb. To use the device, an added attachment as shown in Figure 4.1 (E) could 
be implemented. For healthy individuals, the bicep attachment in Figure 4.1 (F) could be used instead, where the device would be mounted in parallel to the forearm.

\subsection{Compact Controller Design}

The controller system housed the following hardware: two batteries, a microcontroller, and four servomotors (Figure 4.1 (A)). The batteries serve as a portable powerhouse for the servos which were used to drive the Bowden cable system. I designed the hardware to be stored in a remote compact location to help minimize the weight in the residual limb. In the work of Delph et al. their servomotors were similarly stored in a backpack design but were bulky and not practical for a portable prosthetic. The Bowden cables were arranged on the side of the controller and travel directly to the hand. The $3.89 \mathrm{~mm}$ OD and $1.57 \mathrm{~mm}$ ID tubes were fixed at both ends using conduit bulkhead fittings that screw into a fixed point. Running through the tubes was 7x19 cable with a $0.97 \mathrm{~mm}$ diameter stretching the length of the fingertips to the servos.

\subsection{Manufacturing and Hardware}

There were several manufacturing methods that could have been selected to produce the device; however, trading off between availability, cost, speed of manufacturing, and weight, 3D printing was the most viable option. Regarding availability, with 3D printing growing in popularity it is becoming more easily available, this allows the device to be easily reproduced for either personal or commercial use. Product cost and speed vary depending on the size of a component, its weight (in regard to how dense the object happens to be), and complexity. For example, the center palm piece shown in Figure 4.2 cost $\$ 1.59$ to produce. It weighed $51 \mathrm{~g}$ and took $9 \mathrm{hr}$ and $19 \mathrm{~min}$ to manufacture. Despite the longer print times, this method of manufacturing was affordable, durable, and I was able to print with several types of materials. Some parts took more or less time depending on the parameters above. These specifications were based on one material selected, known as 
PETG. Other materials included PLA and TPU. PLA and PETG are more rigid thermoplastics (PETG is more durable though) whereas TPU is a flexible thermoplastic (Ultimate Materials Guide -Tips for 3D Printing with PLA, 2020; Ultimate Materials Guide - 3D Printing Flexible Filament, 2020; Ultimate Materials Guide - Tips for 3D Printing with PETG, 2020). TPU was used in situations such as the fingertips where flexibility could help with grip. PLA and PETG were used interchangeably; however, PETG has its advantages in components where durability is needed.

The finger and thumb movement was controlled using four $1.96 \mathrm{Nm}$ servomotor. All motors and Arduino Mega 2560 microcontroller were powered by two 5000 mAh GFORCE batteries. Additional hardware included the cable and conduit end fittings.

\subsection{Control}

Ideal joint trajectories, as described previously, were used to drive the servomotors. To ensure the system was running as close to real time as possible, a time controller was implemented to properly adapt the command signal to the ideal input signal. There were only four actuators controlling the fingers and thumb with their motion described by the MCP and CMC joint trajectories, respectively, the additional actuators were used to drive the two wrist DOFs. The goal was to drive the device using kinematics; therefore, some conversion was necessary. To use the kinematic inputs, they were first mapped onto the angular ROM of the servo motor pulley. The angle was normalized to the pully limits and then mapped to the motor input limits (between 0-1). Each time a command was sent from MATLAB, the time was recorded. If the time to execute the command was longer than the ideal (reference) signal, then the code would essentially "catch up" to the ideal time. 
One important metric to quantify during the movements was the latency between the command and response signals. The first step to do this was to timestamp the data for both the command signal and the kinematic output signal, over the course of the trial. This process can be seen in Figure 4.4. The data was then synced and for each movement, cross correlation was performed to determine the time delay of the command and output signals. Since there were fewer motors than there were joints, the same command signal was used to calculate the corresponding delay for all joints in a finger. For example, the index finger consists of the MCP, PIP, and DIP joints, and it is driven by one servomotor; therefore, the cross correlation was performed using the same command signal but varying the output signal corresponding to the different joints. Figure 4.5 shows and example of the different start and end times for the ideal, command, and output signals.

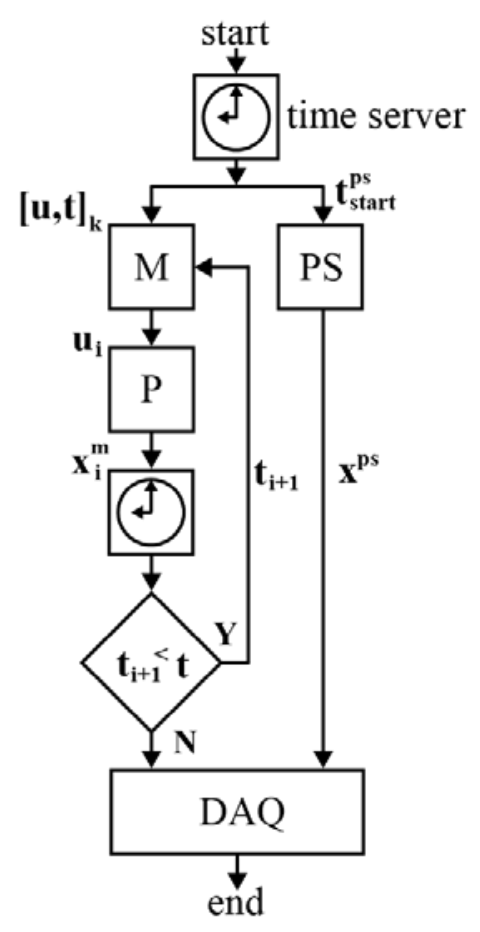

Figure 4.4. Command and response signal recording process. Both signals were recorded on the same time server for the purposes of being able to compare the data and determine the delay between their start times. 


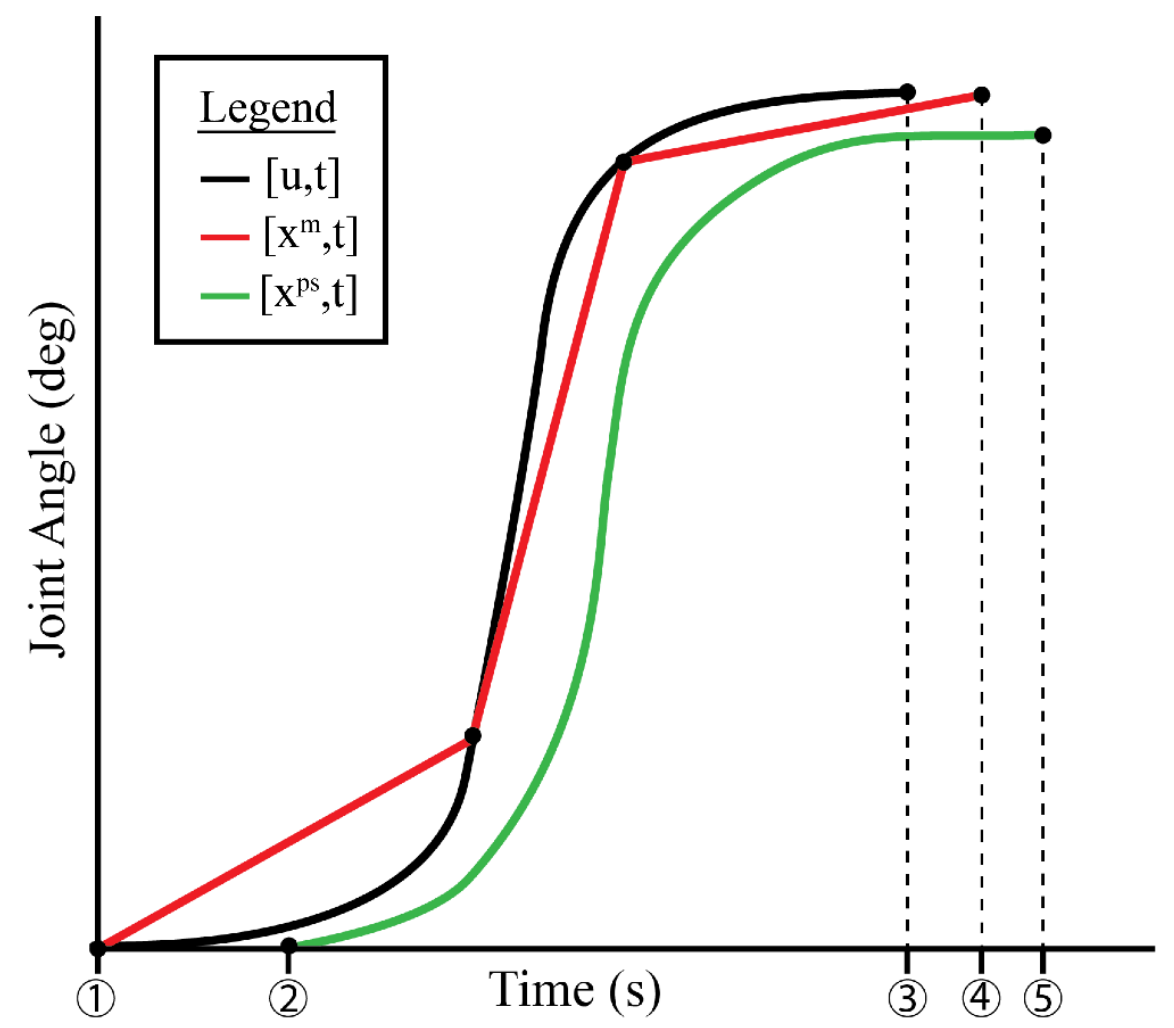

Figure 4.5. Signal delay. 1) Reference/command start time, 2) response start time, 3) reference end time, 4) command end time, and 5) response end time. The difference between the command and actual signal start times can be seen in the plot above. The plot also demonstrates the difference in the joint angles between the ideal, command, and kinematic output signals.

\subsection{Experimental Setup}

\subsubsection{CS Setup}

Kinematics of the robotic hand were compared to the reference and command signals. The hand was instrumented with active LED markers tracked with a motion capture system (PhaseSpace, San Leandro, CA). The minimal set of needed anatomical landmarks was collected to reconstruct digit and wrist DOFs, as shown in Figure 4.6-7. 


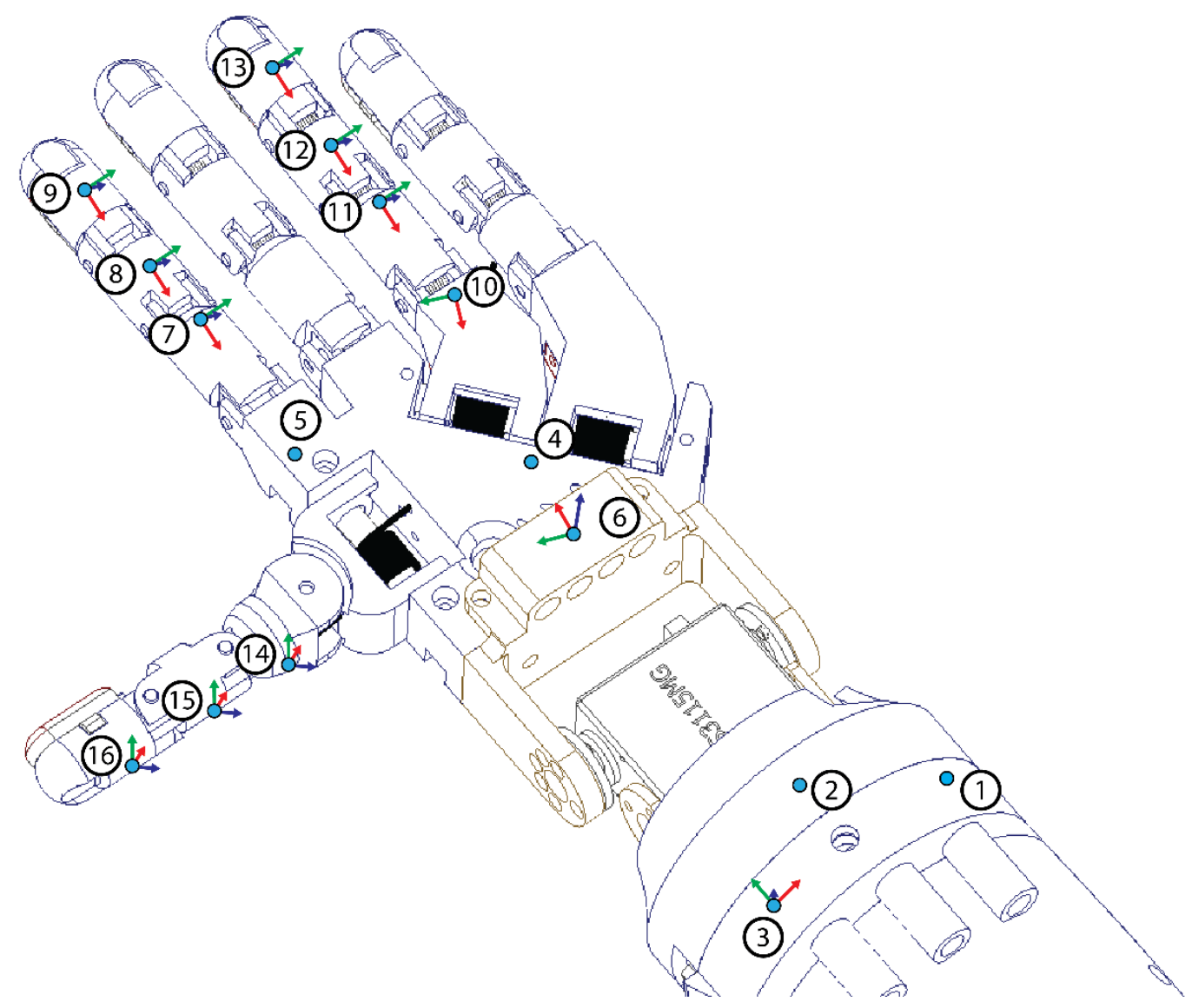

Figure 4.6. LED hand marker layout. This diagram includes the layout of the LEDs (blue circles) as well as the local coordinate systems used to calculate the joint angles. The $\mathrm{x}, \mathrm{y}$, and $\mathrm{z}$ directions are represented by the red, green, and blue arrows, respectively. 

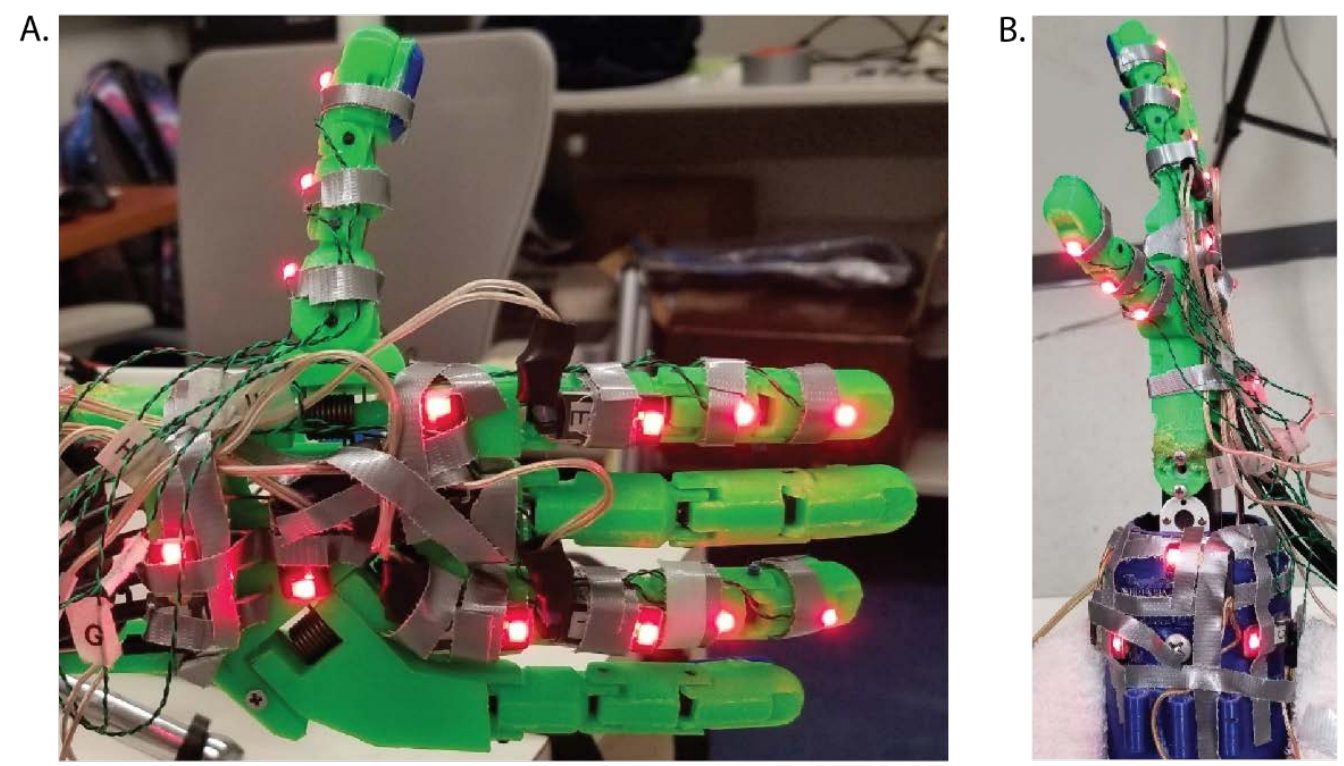

Figure 4.7. Experimental LED marker layout. A. Palm view of markers 4 through 16 . B. Top view of markers 1 through 3.

Local coordinate systems (CS) were established at anatomical landmarks on each rigid body in the kinematic chain. These landmarks were labeled with markers 3, 6, and 7 through 16 . The kinematic chain of segments was setup initially at the forearm (established with markers (1-3)). This segment was rigidly connected on one end, and on the other end there was a two DOF joint separating the wrist. The wrist was established using markers (4-6). There were three additional joints connected to the palm including the thumb, index, and ring fingers, each 1 DOF. The thumb and ring were made up of four segments with three additional joints. The last link in the kinematic chain was the distal segment for each of the digits. The index finger, unlike the other digits, only had three segments with two additional joints. With the exception of the forearm, wrist, and first digit segments, the coordinate systems of the digits were constructed using the most proximal segment. Figure 4.8 shows an example of how the coordinate systems were constructed by knowing points on the proximal segment. 


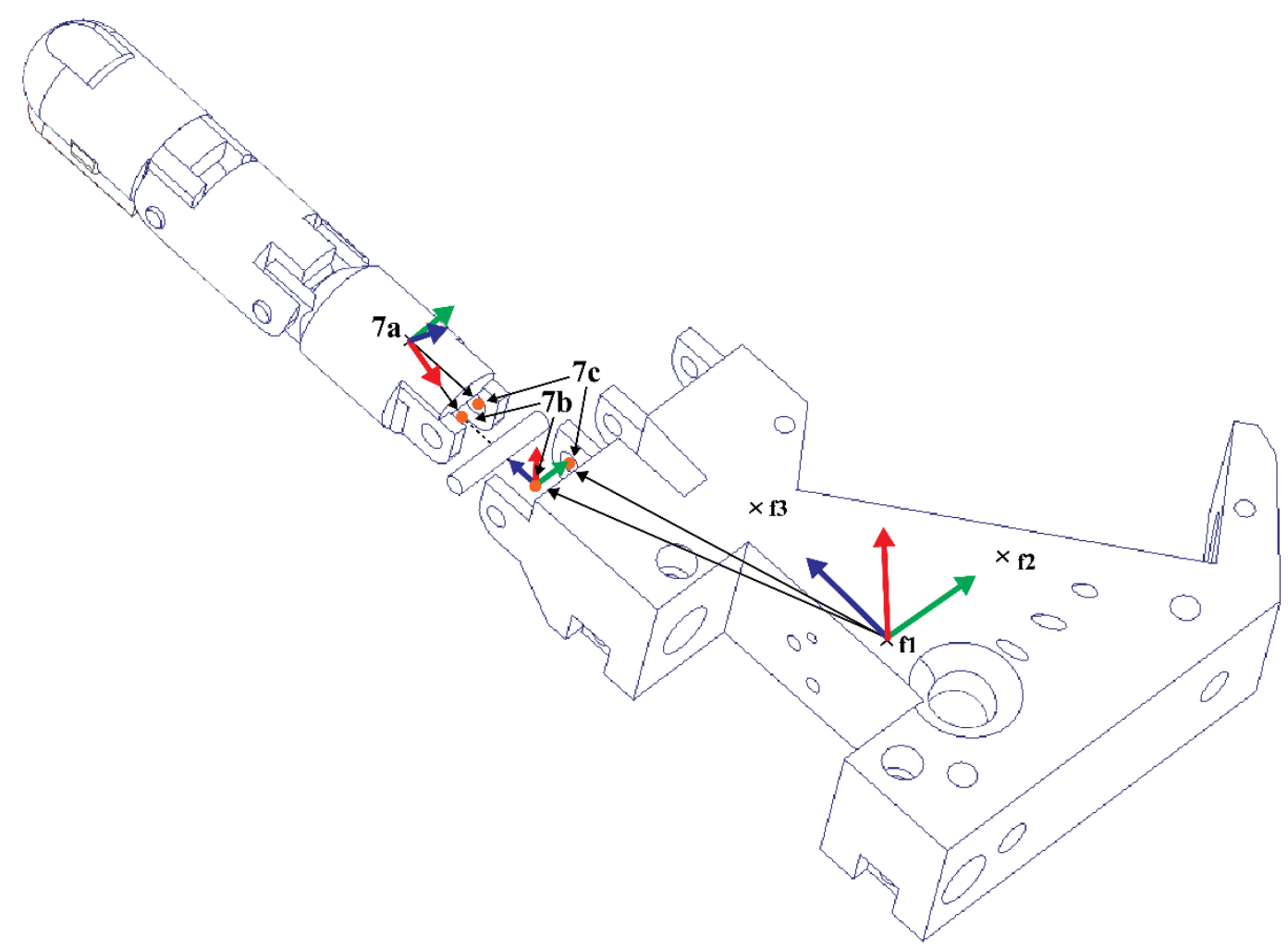

Figure 4.8. Local coordinate system setup. Local coordinate systems were established relative to the more proximally defined coordinate systems, except the CS at markers 3 and 6.

Most of the finger CSs, including the one at marker 7 in Figure 4.8, used the following method to generate the local $\mathrm{x}, \mathrm{y}$, and $\mathrm{z}$ axes. Using the local CS at marker 6, the distances to points $\mathrm{f} 1, \mathrm{f} 2$, f3, 7b, and 7c were measured. To convert these local points into global points, it was necessary to follow the process shown in Appendix C.1. Here, a specific example for transforming a point in CS 6 to the global CS is provided. To convert the points to their global form, the rotation matrix from CS 6 to the global CS was calculated, as well as the coordinates of the point in local coordinates, and global coordinates of CS 6's origin. With the CSs established at local joints, the next step was the calculation of joint angles using the method described in Research Methods in Biomechanics detailed in Appendix C.2 (Robertson et al., 2013). The joint angles were used in the 
upcoming sections to determine the error in performance between an ideal hand and the new design.

\subsection{Equipment Setup}

Eight motion capture cameras were setup in an arc shape to record hand kinematics. Figure 4.9 shows four of the floor cameras. The cameras captured data at $480 \mathrm{~Hz}$, this was later filtered using a $6 \mathrm{~Hz}$ low-pass second order Butterworth filter to smooth the kinematic data.

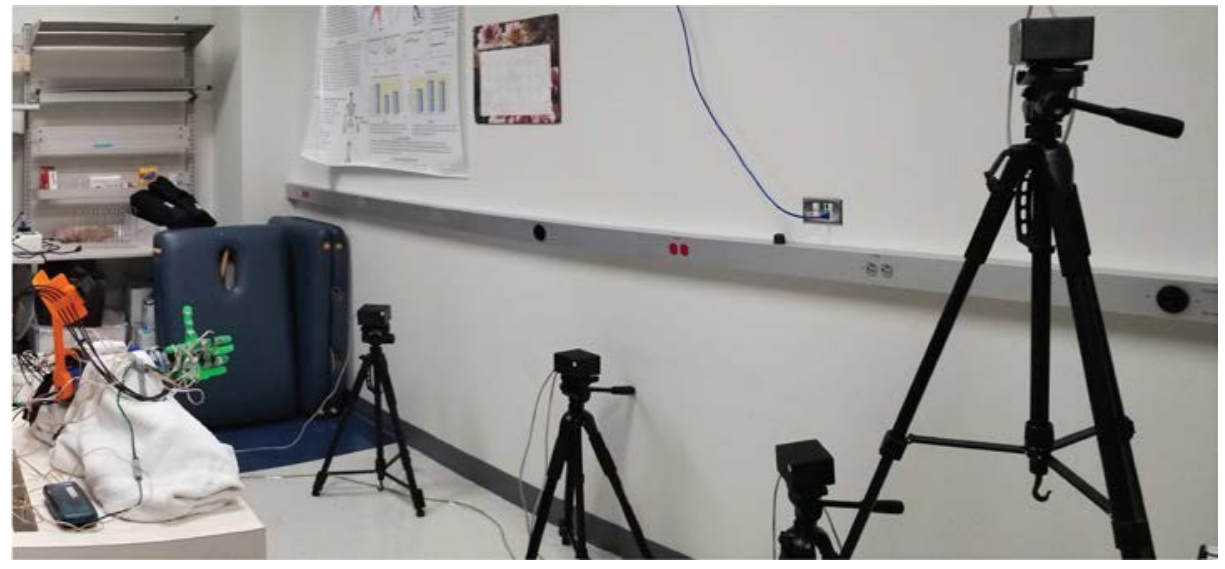

Figure 4.9. Camera setup. The motion capture cameras were oriented to best capture the LED hand and forearm markers. Some were placed below the height of the table, slightly above, and even higher on the ceiling.

The hand and forearm were oriented in a neutral position; however, they were slightly angled off the floor. This was done to allow the LEDs to be seen better by the cameras. Due to the lack of available markers, the movement of the middle, ring, and small finger was evaluated using only the movement of the ring finger (as it is the center of the coupled fingers). Also, the thumb did not have enough markers to directly measure the movement for both DOFs. To obtain the joint angles for both the flexion-extension and abduction-adduction movements an assumption made was that the normal to the plane, made up by the 3 thumb markers (14-16), was parallel to the palm normal. The angle between these two normals was the thumb abduction-adduction angle. To prevent 
translational motion, the forearm was clamped and placed on a stationary table (shown in Figure 4.10). The controller was also fixed to the table. By securing both ends of the system, the Bowden cable would properly slide within the inner tubing to open and close the fingers. A total of 10 movements, listed in Table 4.1, were performed 10 times each. Movements 1-6 were explicitly shown with an associated CAD drawing. The remaining movements 7-10 were grouped together to represent the extension position, meaning that if a digit was to flex and then extend, this is what it would look like fully extended.

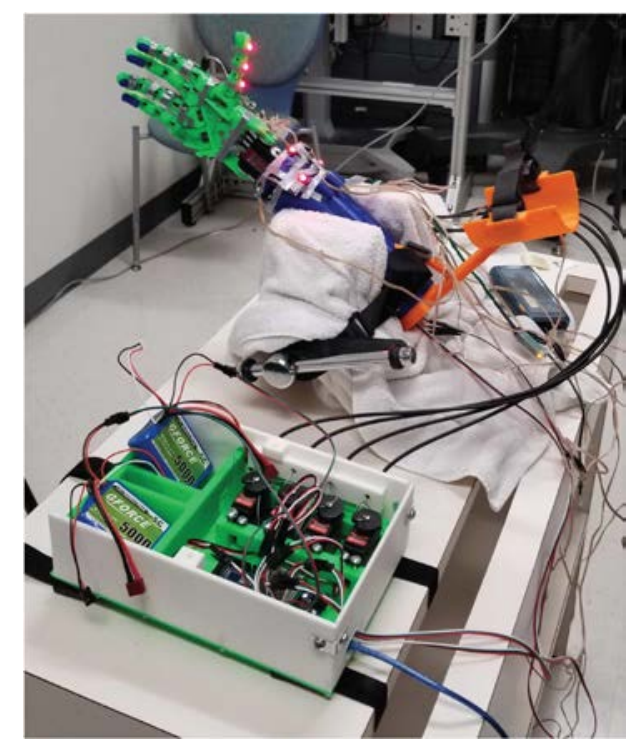

Figure 4.10. Hand and controller system setup. The forearm and hand were fixed and angled to be in better view of the cameras. The controller system was also strapped to the table to prevent unwanted movement. 
Table 4.1 Movement tasks. There were 10 movement tasks performed. Each tested an individual DOF through the entire range of motion.

\begin{tabular}{|l|}
\hline Movement Task \\
\hline 1) Index Flexion \\
2) Thumb Flexion \\
3) Thumb Adduction \\
4) Middle, Ring, Small \\
Finger Flexion
\end{tabular}




\section{Chapter 5: Results}

\subsection{Overview}

The results for the simulation are presented below within the following two sections: simulation results and experimental results. Simulation results were tailored toward the Simulink model whereas the experimental results focused on the latency and joint angle RMSE calculations.

\subsection{Simulation Results}

The data was recorded for a total of 1200 trials. The trials were grouped into two categories based on their step size: $10^{-3}$ and $10^{-5} \mathrm{~s}$. The normalized root mean square error (NRMSE) was used to quantify the error of the output torque between the step sizes of $10^{-3}$ and $10^{-5} \mathrm{~s}$. The trials were grouped into wrist flexion-extension and pronation-supination with a total of 300 NRMSE values generated for each wrist DOF. The error between the step sizes was found to be zero with at least $10^{-12}$ accuracy between the two DOFs tested. Once it was determined that the solution was

numerically stable, Table 5.1 was produced using $10^{-3} \mathrm{~s}$ values, reflecting the worst-case scenario torques experienced under different conditions. The table was organized by movement type, DOF, spherical mass, rotational speed, posture ID, minimum torque, and maximum torque. The first was movement type: this means the wrist is flexing, extending, pronating, or supinating. This was followed by the DOF in regard to where the wrist torque was measured, either at the flexionextension or the pronation-supination DOF. The next category was the spherical mass, which was the additional mass the hand had to hold, followed by the maximum rotational speed. The posture ID describes the posture of the hand and the angle of the non-moving wrist DOF. Finally, the last two categories include the minimum and maximum wrist torques at the respective DOF measured. The first four rows corresponded to the overall maximum and minimum torques experienced regardless of the rotational speed and loading (although the conditions turned out to be the same 
for all four cases). The remaining rows produced torques that were based on a specific rotational speed and loading condition: $60 \mathrm{deg} / \mathrm{s}$ with either a 1 or $2 \mathrm{~kg}$ mass. On the lower end of the spectrum with an absolute minimum torque of $0.0041 \mathrm{Nm}$ was when the hand was positioned horizontally with the wrist flexed at an angle of 0.7418 radians. The wrist was undergoing pronation-supination movement and the torque was measured at the flexion-extension DOF, with a mass of $1 \mathrm{~kg}$ and a rotational speed of $60 \mathrm{deg} / \mathrm{s}$.

Table 5.1. Simulated torque data for hardware selection. The table is broken down into several categories including movement type, DOF, spherical mass, rotational speed, posture ID, minimum torque, and maximum torque.

\begin{tabular}{|c|c|c|c|c|c|c|}
\hline Posture ID & Movement Type & DOF & Mass (kg) & Speed (deg/s) & Min Torque (Nm) & Max Torque (Nm) \\
\hline 1 & FE & FE & 6 & 120 & -4.08 & 3.89 \\
\hline 2 & FE & PS & 6 & 120 & -3.92 & 3.74 \\
\hline 3 & PS & PS & 6 & 120 & -4.14 & 0.69 \\
\hline 4 & PS & FE & 6 & 120 & -3.97 & 0.15 \\
\hline 5 & FE & FE & 2 & 60 & -0.84 & 1.40 \\
\hline 6 & FE & PS & 2 & 60 & -1.38 & 1.31 \\
\hline 7 & PS & PS & 2 & 60 & -1.45 & 0.19 \\
\hline 8 & PS & FE & 2 & 60 & -1.39 & 0.03 \\
\hline 9 & FE & FE & 1 & 60 & -0.76 & 0.71 \\
\hline 10 & FE & PS & 1 & 60 & -0.74 & 0.70 \\
\hline 11 & PS & PS & 1 & 60 & -0.78 & 0.10 \\
\hline 12 & PS & FE & 1 & 60 & -0.75 & 0.00 \\
\hline
\end{tabular}

Posture ID: $1=$ hand pointing vertically up; $2=$ hand horizontal and wrist pronated-supinated at 0 rad; $3=$ hand horizontal and wrist flexed at $1.3963 \mathrm{rad} ; 4=$ hand oriented diagonally between horizontal and vertical and wrist flexed at $0.7418 \mathrm{rad}$;

$5=$ hand horizontal and wrist supinated at $1.3963 \mathrm{rad} ; 6=$ hand horizontal and wrist pronated-supinated at 0 rad;

$7=$ hand horizontal and wrist flexed at $1.3963 \mathrm{rad} ; 8=$ hand horizontal and wrist flexed at $0.7418 \mathrm{rad} ; 9=$ hand horizontal;

$10=$ hand horizontal and wrist pronated-supinated at $0 \mathrm{rad} ; 11=$ hand horizontal and wrist flexed at $1.3963 \mathrm{rad}$;

$12=$ hand horizontal and wrist flexed at $0.7418 \mathrm{rad}$.

In addition to the results regarding hardware selection, the simulation data was also verified with the analytical calculations. The resulting plots are shown in Appendix D. Movement 1 data can be seen in panels (A-C), and includes the motion profiles for the position, velocity, and acceleration, followed by the horizontal and vertical movement of the COG, and lastly the torque comparison for the analytical and numerical solutions. Similarly, movement 2 data is shown in panels (D-F) 
and movement 3 data is shown in panels (G-I). The local CS of the of the hand was established with the positive $x$-axis pointing from medial to lateral, the $y$-axis pointing from the anterior palm to the posterior palm, and the z-axis pointing from proximal to distal along the arm segment. This means that the torque corresponding to flexion-extension was calculated about the $\mathrm{x}$-axis, and the torque corresponding to pronation-supination was calculated about the z-axis. The normalized RMSE for each movement is shown in Table 5.2. The smallest errors were seen in movement 1 about the $\mathrm{z}$ axis and the largest error was seen in movement 2 about the $\mathrm{x}$ axis.

Table 5.2. Normalized RMSE values. These values represent the normalized RMSE between the analytical and numerical torque data.

\begin{tabular}{|l|l|l|l|}
\cline { 2 - 4 } \multicolumn{1}{c|}{} & Movement 1 & Movement 2 & Movement 3 \\
\hline Error X Axis & 0.0070 & 0.0151 & 0.0075 \\
\hline Error Z Axis & 0.0028 & 0.0113 & 0.0094 \\
\hline
\end{tabular}

\subsection{Experimental Results}

The latency was calculated between the command and response signals which can be seen in Figure 5.1 below. The figure was grouped into two examples with latencies of $\geq 200 \mathrm{~ms}$ and $\geq$ 400 ms. The example movement corresponding to the 200 ms delay was for wrist flexion Figure 5.1 (A \& C) and the movement corresponding to the 400 ms delay was for thumb abduction of the CMC joint Figure 5.1 (B \& D). Figure 5.1 (A) shows a comparison between three signals; however, the comparisons of interest are between the reference signal (black) and the command signal (red) as well as the command signal and the response signal (blue). A total of 10 command signals were sent to the servo motors. The last command signal extends beyond the length of the reference signal and represents the final signal sent to the motor. It can be seen that the response signal does not start moving for about $200 \mathrm{~ms}$ after the first signal was sent from the motor, but this is more 
prominently seen in Figure 5.1 (C). This panel shows the delay of the reference signal, $200 \mathrm{~ms}$, at an associated unbiased correlation value (or signal similarity measurement) of 0.47 . Similarly, in Figure 5.1 (B), the reference and command signals were compared, except this time there was one less command signal sent to the motors compared to the wrist flexion motor. The number of signals sent could change depending on how fast the motor was able to catch up to the reference value. The difference in the start times between the command and reference signals was even larger, measuring $360 \mathrm{~ms}$, as shown in panel (D). The associated unbiased correlation value was 0.49 , showing that the signals were more similar the measured delay value for the thumb CMC joint than they were for the wrist flexion joint at its corresponding delay value. It should be noted that the command and reference signals pictured in Figure 5.1 were the original signals and were not used for the cross-correlation calculation. Cross-correlation was performed between the response signal and the interpreted command signal such that the signals were equal in length. The start and end times remained the same for the command signal as shown in Figure 5.1; however, the number of points between increased. 

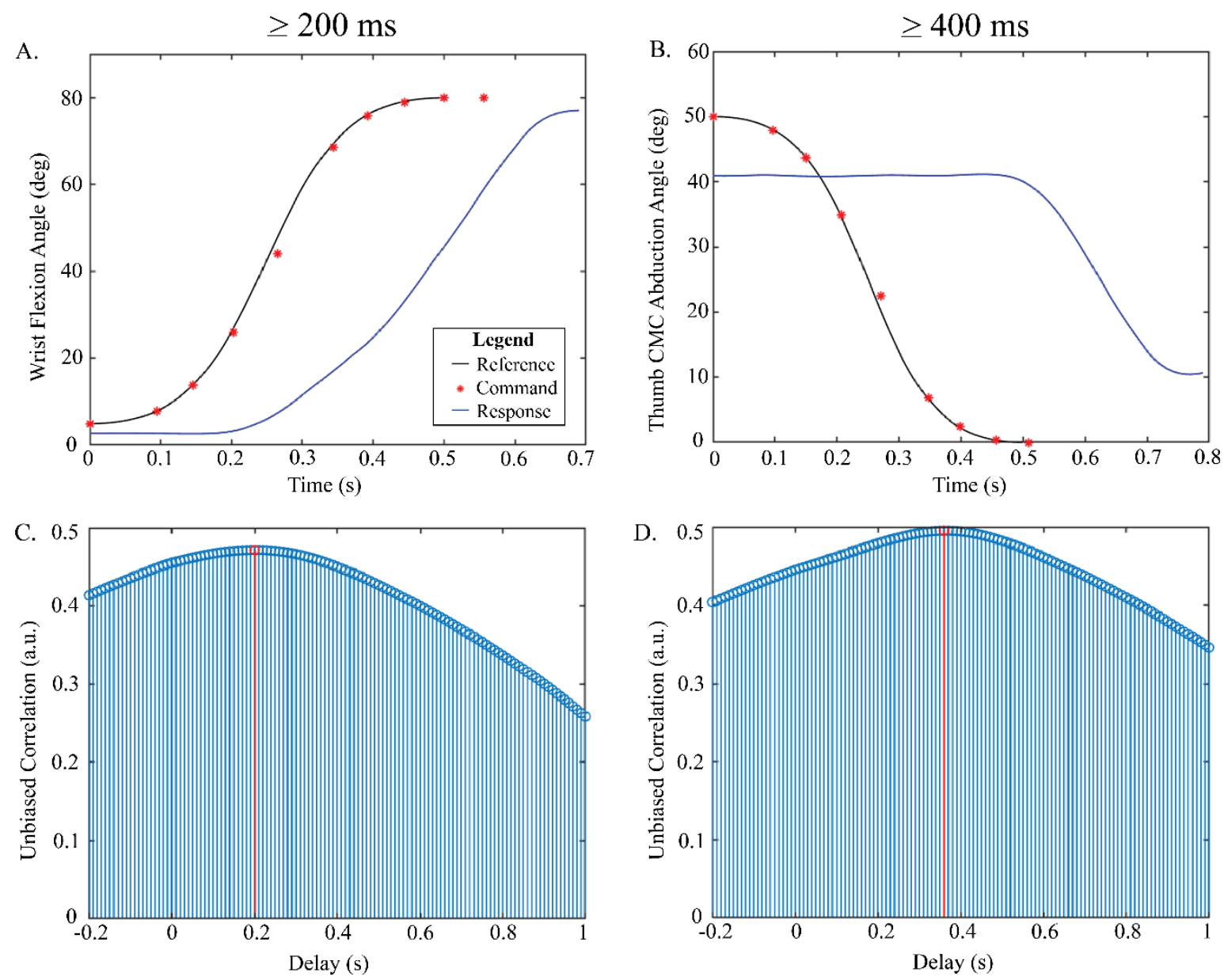

Figure 5.1. Examples of signals with representative delays. A. Index flexion at the MCP joint plotted with the reference, command, and response signals. B. Delay measured in seconds and the associated unbiased correlation value for the index MCP joint. C. Thumb abduction at the CMC joint plotted with the reference, command, and response signals. D. Delay measured in seconds and the associated unbiased correlation value for the thumb CMC joint.

Using cross-correlation, the delays were calculated for all 100 trials across the 16 DOFs and presented in a boxplot (Figure 5.2). Along the $x$-axis are the DOFs (where a description is provided in Table 5.3), and along the $y$-axis are the corresponding delays. The delays are grouped into two categories, $200 \mathrm{~ms}$ and $400 \mathrm{~ms}$ delays. The smaller delays were seen among most of the index 
finger, wrist, and a few thumb movements. The delays for each of these boxplots was not widely distributed and, in most cases, the mean value was equal to the upper quartile (or lower quartile), meaning most of the data fell into this upper section (or lower section) of the distribution. It can be seen that the remaining index, thumb, and all ring finger movements were more widely distributed in delay values thus showing less consistency of delays between trials testing the same movements. The servo motors were directly connected to the joint in which they were controlling the two wrist DOFs, and their data was relatively consistent between trials. The remaining DOFs were controlled using the Bowden cable system which can introduce more variability into the system. Since the delays were measured between when the command signal was sent and when the movement was recorded using motion capture, possible reasoning for this variability in delays could be more of a mechanical issue specifically regarding the cable system and how it was setup. The pulley not wound tight enough could have caused the motor to spin but taken longer before the cable was taught and the finger started moving. There will also be a small delay contribution, regardless of the mechanical performance, due to the time required to send the command signal from MATLAB to the servomotors. In addition to calculating the execution delays across the DOFs, the RMSE values were also determined. 


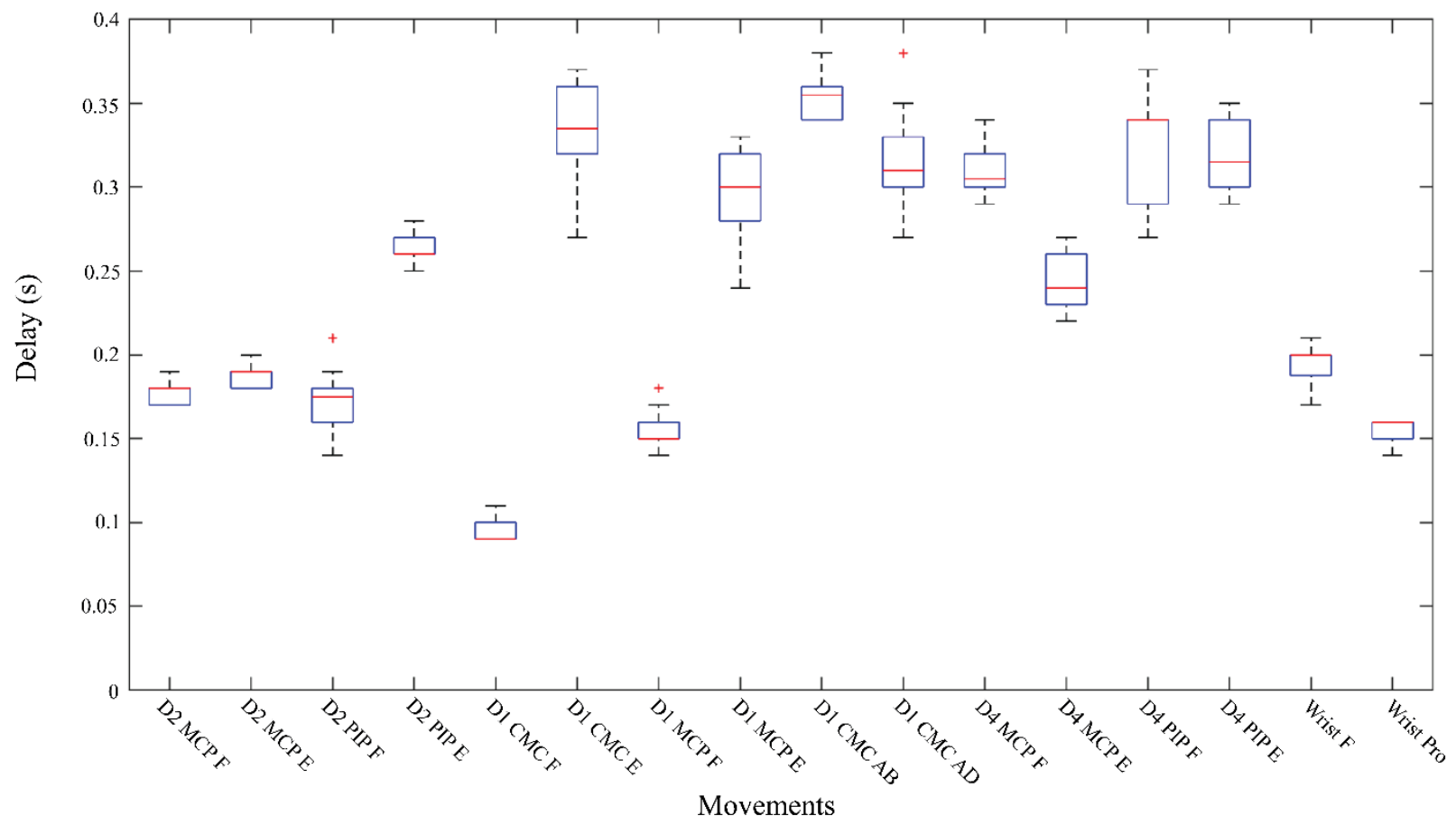

Figure 5.2. Signal delays across movements. The average delay between each command signal and the respective joint that is presented above. The servo delays were averaged for the 10 trials within the respective movement. 
Table 5.3 Description of DOFs. This table provides a more detailed description of the DOFs presented in Figure 5.3.

\begin{tabular}{|l|l|}
\hline DOF & Description \\
\hline D2 MCP F & Index MCP DOF during Flexion \\
\hline D2 MCP E & Index MCP DOF during Extension \\
\hline D2 PIP F & Index PIP DOF during Flexion \\
\hline D2 PIP E & Index PIP DOF during Extension \\
\hline D1 CMC F & Thumb CMC DOF during Flexion \\
\hline D1 CMC E & Thumb CMC DOF during Extension \\
\hline D1 MCP F & Thumb MCP DOF during Flexion \\
\hline D1 MCP E & Thumb MCP DOF during Extension \\
\hline D1 CMC AB & Thumb CMC DOF during Abduction \\
\hline D1 CMC AD & Thumb CMC DOF during Adduction \\
\hline D4 MCP F & Ring MCP DOF during Flexion \\
\hline D4 MCP E & Ring MCP DOF during Extension \\
\hline D4 PIP F & Ring PIP DOF during Flexion \\
\hline D4 PIP E & Ring PIP DOF during Extension \\
\hline Wrist F & Wrist Flexion DOF \\
\hline Wrist P & Wrist Pronation DOF \\
\hline
\end{tabular}

The RMSE values were calculated using the reference and response joint angles. Figure 5.3 (A) shows an example of the movement "Wrist flexion". Both signals have been aligned starting at 0 , factoring in the delay. The resulting wrist flexion RMSE value was found to be $8.6 \mathrm{deg}$. It can be 
seen that the signals start to deviate from one another between the 0.2 and 0.5 second interval. Both show an increase in joint angle and reach close to the same peak value; however, the response signal does not follow the ideal path of the reference signal. One possible reason for this was due to the command signal being approximated when mapped to the ideal inputs. This could have caused the profile to be skewed since the mapped ROM was projected to be 80 deg, but as shown, the response signal did not reach that value.

In Figure 5.3 (B), the reference and response signals were plotted for the thumb CMC abduction movement. Starting off, the abduction angle was below the maximum reference angle; however, the signals crossed around the $0.2 \mathrm{~s}$ level but began to diverge toward the end of the movement, until the response signal steadied around 11 deg. The RMSE value for this movement was 7.3 deg. Although some of the error for this joint was due to the mechanics, visually during the experiment, the thumb traveled through the entire ROM. The most likely reason that the plot does not show a value closer to 0 at $0.5 \mathrm{~s}$ for the response signal is to how the thumb joint angle was calculated. The thumb abduction-adduction ROM was affected by the necessary calculations to obtain the flexion-extension plane of the thumb. Due to experimental marker placement, certain positions during the movement caused the markers to be aligned; therefore, the plane was no longer able to be calculated. To account for this during the abduction-adduction, the normal of the thumb flexionextension plane was calculated at a point in time during flexion-extension since it remains constant during the movement. Taking the known global flexion-extension normal vector and knowing that this is the same normal that would occur at the first instance of abduction-adduction, the normal was rotated into the thumb local CS for each point over the movement. That being said, if the thumb normal vector was off at all from the exact parallel normal to the palm, this will cause error in the subsequent thumb joint angle calculations. 

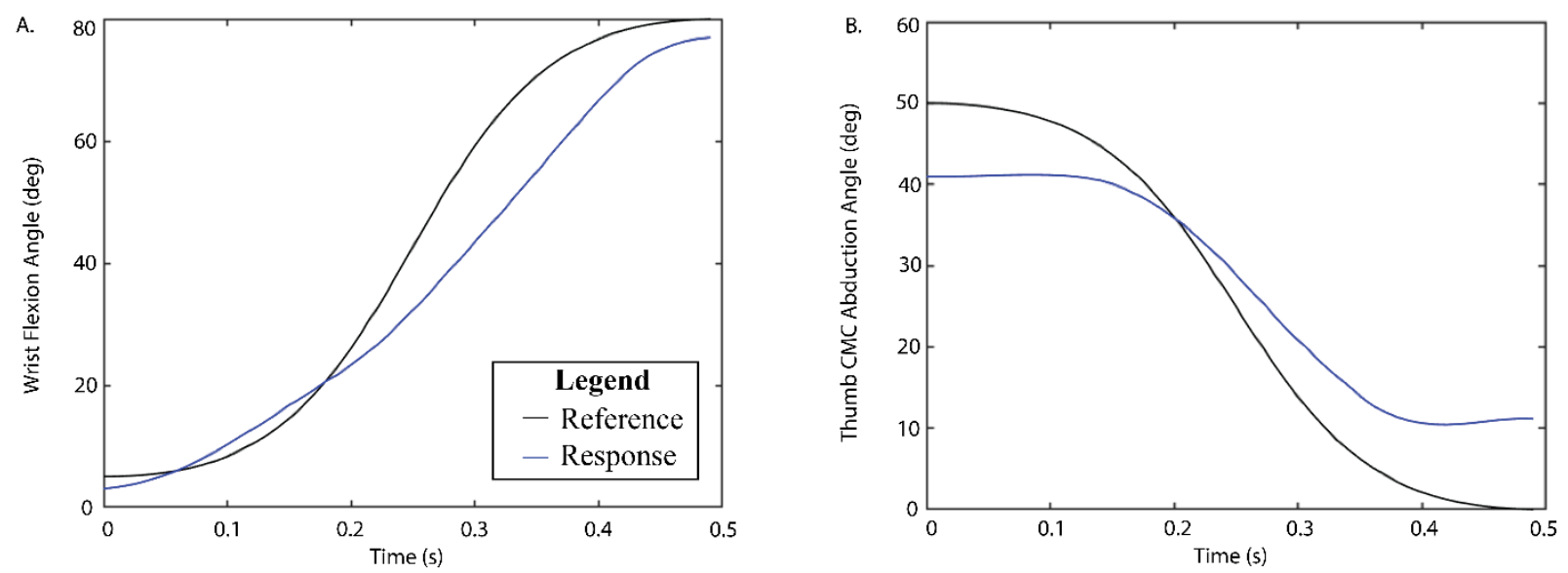

Figure 5.3. Example RMSE value plots. A. Reference, command, and response signals used to generate the RMSE value for the index MCP joint. B. Reference, command, and response signals used to generate the RMSE value for the thumb CMC joint.

The RMSE values for all trials were presented with a boxplot, as shown in Figure 5.4. The interquartile ranges were very small for all but a few of the distributions. It is also noticeable that the whiskers are either small or only on one side of the data as well for most of the data. The RMSE values were not widely spread and tended to be less scattered and more consistent across trials. The smallest error was exhibited in the thumb CMC joint during flexion was approximately $2^{\circ}$ with the largest error at $55^{\circ}$, found in the ring PIP joint during flexion. The average RMSE across all joints was $19^{\circ}$. One important piece of information to note from this figure is that the mechanics (ROM) for the PIP joint was similar across all fingers. It does not appear this way from the figure though considering the median of the index PIP and ring PIP joints during flexion are nearly 45 deg, and the thumb median RMSE value for the MCP joint is under $5^{\circ}$. [Although the names are different, the thumb MCP and index/ring PIP joints have the same placement (second joint from distal segment) in the digits]. Further investigation revealed that based on the segment design, the joint had approximately a $40^{\circ}-50^{\circ}$ ROM capability. When comparing this ROM to maximum 
ROM for the respective joints, the error could vary significantly. Both the index and ring PIP ideal joint ROM is $120^{\circ}$ whereas the thumb ROM is only $45^{\circ}$ leading to large differences in the RMSE values for these joints. Similarly, this happened with the extension movements of those same joints. There was not a wide distribution of data for most of joints, the thumb CMC joint during flexion, ring MCP joint during extension, and ring PIP joint during flexion had the most variation among trials of the same movement. The wrist DOFs were very similar in RMSE values most likely due to alignment as the motors were more than capable of covering the full ROM for the joint.

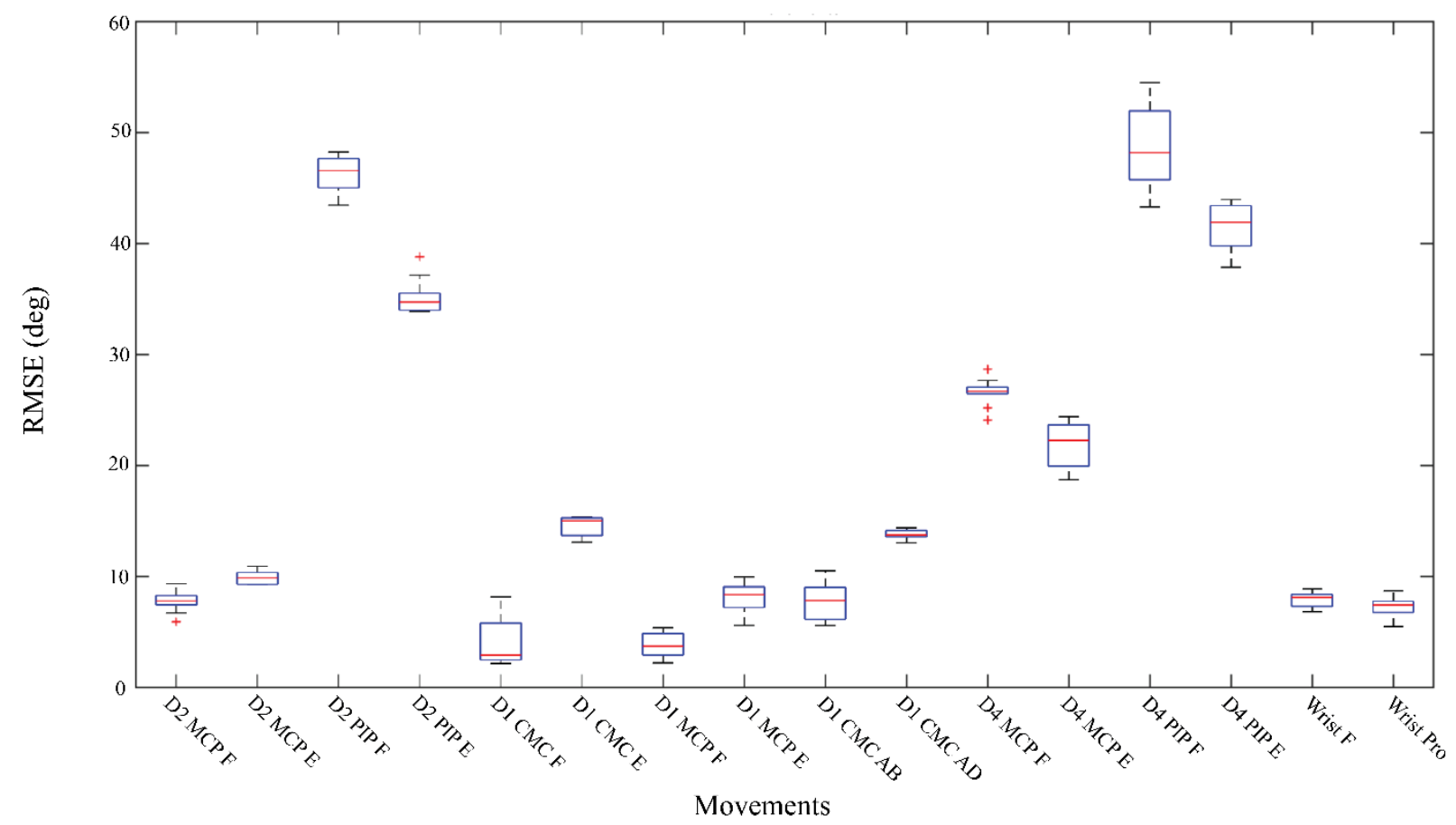

Figure 5.4. RMSE values across movements. The RMSE value for all movements testes is presented above. 


\section{Chapter 6: Discussion and Conclusions}

\subsection{Discussion}

In this study, an additional DOF was added to an existing robotic arm. Simulations were initially performed to determine hardware requirements for the added wrist flexion-extension DOF as well as the wrist pronation-supination DOF. Modifications were made by adding wrist flexionextension, combining the middle, ring, and small fingers, as well as adding a controller system. Following the manufacturing of the device, kinematic movement was demonstrated for a variety of individual movements. This section discussed the reasoning behind the design modifications that were made, data analysis regarding the simulated and experimental testing, the overall limitations, future work, and concluding thoughts.

\subsubsection{Design}

There were several designs were considered. These included individual finger control for all fingers, reducing the hand to only three or four fingers to mimic more of a tripod gripper, or possibly even only having a one DOF thumb and wrist. The reason that the hand was specifically designed with a two DOF wrist and thumb, individual index finger control, and coupled middle, ring, and small fingers was to maximize function without encroaching on space limitations. The index finger was designed this way to mimic human grasping, as it is the most independent of the four fingers (Häger-Ross \& Schieber, 2000; Kilbreath \& Gandevia, 1994; Mason et al., 2001). For similar reasoning the thumb was designed in this manner as it accounts for nearly $40 \%$ of hand function (Weir, 2005b, p. 32.32). One study even demonstrated that a 2-DOF thumb in a 4-DOF hand was comparable to a 23-DOF hand during most grasps tested (Montagnani et al., 2016). Although the thumb was not modified significantly from the original design, the two DOF capabilities allowed for precise grasping when used with the index finger. 
In terms of the wrist, although the specifications of the motors were not known to start, one design constraint was implemented, and that was the use of a low-profile servo for the pronationsupination DOF. The purpose of this was to provide a compact design and reduce the overall length of the forearm such that the device would be as close to the length of the natural human arm as possible. The pronation-supination DOF combined with the flexion-extension DOF design has been implemented in a similar work by Olsen et al.; however, this was only an attachment for existing prosthetics and did not address where the additional motors would go for driving the hand (Olsen et al., 2019). Our solution not only has this additional DOF along with pronationsupination, but it also relocates the motors to a controller system to reduce the weight on the residual limb. In a similar work by Delph et al., a rehabilitation glove was used to help patients move their fingers; however, the design was based on a controller system that used servomotors to distally actuate the fingers from an exoskeleton perspective. Although a similar method was used to actuate hands from distal locations, our design and implementation is to be used with prosthetic applications (Delph et al., 2012). This requires the design and development of not only a controller, but the prosthetic hand and forearm.

The prosthetic hand and its layout have been discussed, but equally important was the design of the Bowden cable system and how it interacts with the hand. Although a pull system with a torsional spring inserted in the fingers was the design chosen, other systems were considered including gear, pull-pull, and push-pull. Gear systems for example, have the benefit of creating a compact design by locating the motors proximally in the fingers; however, the location can cause added weight to the device and smaller motors can reduce the amount of grip force available (Krausz et al., 2016). The advantage to pull-pull systems is their ability to produce a strong flexion and extension force and are generally only subject to deflection within the cable system 
(Steuernagel). The downside is that they require two cables per finger actuated. The push-pull system is beneficial because it can reduce the number of cables needed in half, compared to a pullpull system; however, the cable must be rigid enough to be pushed while also maintaining flexibility for prosthetic applications. In addition to this, it is subject to both backlash and deflection reducing the efficiency of the system. A compromise between these systems was the integration of torsional springs into the finger joints. The springs take care of the push factor by restoring the fingers to an extended position, while the cables pull cause the fingers to flex and grasp around an object. The cable system allows for multiple joints to be actuated at the same time and from the same actuation source; for example, the driving joint (i.e., MCP or CMC) is able to move through its $\operatorname{ROM}(\theta)$ and the follower joints (i.e., DIP and PIP) move as a percentage of the driving joint $(0.72 \theta)$ and $(0.23 \theta)$, respectively (Ramirez et al., 2019a). The specific springs selected for the project were based on calculating the torque needed to lift the 3D printed fingers into an extended position against gravity. The calculation did not account for the resistance of the cable, lining the fingers, that the spring would have to also work against to restore the fingers. Instead, a few springs were selected and tried based on the inner diameter (how well they fit within the fingers) and a torque minimally above the spring torque calculated. The spring would not only have to be able to lift the finger, but also pull the cable which was subject to friction. The wind direction of the spring (left or right-handed) was also chosen based on the specific space requirements as the direction would have caused the leg length to be shortened.

Despite the benefits of Bowden cable systems including their ability to actuate from a distance, they do have some potential drawbacks. One of these design considerations or trade-offs that arise is deflection (less so backlash as this is more of a concern with push-pull systems). Minimizing the deflection was considered by fixing the conduit near an anticipated bend i.e. around the 
elbow/bicep etc. and reducing the bends where necessary (Steuernagel). Reducing the bends can also help reduce friction and improve the efficiency (Steuernagel). One way the bending was reduced was by running the tubing out the side of the controller system rather than from the top and down the shoulder (Nycz et al., 2016). Although there were many options to actuate the fingers, the option with the most benefits and fewest drawbacks was the pull cable system paired with the torsional springs.

During testing, it was noted that the thumb flexion-extension servomotor began to fail. The potential cause for this was due to the constant tension on the thumb for $4+$ hrs. The load combined with the prolonged duration of servomotor usage caused overheating. This motor was replaced, and the experiment did continue as planned. Also, regarding the thumb, due to maker placement, they ended up aligning during the experiment. This required additional calculations to create a plane that would be useful to calculate the thumb angles.

\subsubsection{Data Analysis}

To clarify the results presented in Chapter 3 for the simulation and experimental testing, I will discuss them in further detail. Due to no noticeable difference seen between the step sizes checked, it was found that the step sizes evaluated did not affect the accuracy of this solution. That being said, due to computation time, the $10^{-3} \mathrm{~s}$ results were used. The simulation results showed the largest torque at an absolute value of $4.0795 \mathrm{Nm}$; however, this torque is not typically encountered performing everyday tasks such as drinking a cup of coffee, brushing your teeth, or buttoning your shirt. To better fit within the scope of the experiment, while still providing enough torque to be useful, the servomotor selected for pronation-supination was a $1.18 \mathrm{Nm}$ low-profile servomotor. The servomotor chosen for flexion-extension was rated at $1.96 \mathrm{Nm}$ of torque. The low-profile servomotor allowed for a compact design and the flexion-extension motor had a built-in U-mount 
to allow the hand to rotate about the motor. Both motors would be more than capable of completing tasks with a $1 \mathrm{~kg}$ mass moving at $60 \mathrm{deg} / \mathrm{s}$. For studies that would want to test conditions outside of these, it would be recommended to change the motors depending on the testing performed with the arm.

In terms of the simulation verification, the smallest NRMSE values were seen during conditions in which the weight was $6 \mathrm{~kg}$, and the larger NRMSE values were seen when the weight was $1 \mathrm{~kg}$. This could be attributed to assuming all the weight acted at the COG even for the hand itself despite only weighting $\sim 0.2 \mathrm{~kg}$. The issue with that is $0.2 \mathrm{~kg}$ is only $3.3 \%$ of $6 \mathrm{~kg}$ whereas 0.2 is $20 \%$ of $1 \mathrm{~kg}$. By neglecting the actual geometry of the rectangular shape and only considering the moment of inertia generated by the sphere with the added hand weight, this could cause the error to be higher at smaller weights.

In Chapter 4, the error between the ideal and output joint angles was quantified using the RMSE values. The average RMSE value was 19 deg across all joints, with the best and worst values spanning 2 to 55 deg (MCP and third digit PIP DOFs, respectively). In addition to the reasons discussed above, possible additional reasons attributing to the larger errors in the middle, ring, and small finger combination was the design of the Whipple tree mechanism. Increasing the length of the cables for the ring and small finger would have helped to increase the extension ROM. Also providing more room for the cable connection to slide and not get caught during the pulling motion would have improved the flexion as well. Despite the inaccuracies among the finger joints, the wrist joints both showed high accuracy (RMSE $<9)$ and small delays $(\leq 200 \mathrm{~ms})$.

Regarding the latency, the smallest delays occurred in the index finger, wrist flexion, wrist pronation, and some thumb movements, where larger delays (close to $360 \mathrm{~ms}$ ) were seen in the remaining thumb and ring finger joints. Based on the mapping technique used to take kinematic 
inputs and produce servo movements as the output, additional error was introduced. This error caused the delay for the output signal to vary over the course of the movement rather than be more constant. Had the inputs been mapped through the entire ROM of the joint with mechanical limitations implemented, rather than underestimating the capable ROM, less error would have occurred.

\subsection{Limitations and Mitigations}

\subsubsection{Simulation}

Some of the limitations to the simulation in Chapter 3 include only actuating the wrist DOFs. The model would have to be adapted to test other grasping postures and the torque required to actuate other DOFs. Also, the rotational speed and weight selections were limited. To better account for the human wrist, the rotational speed could be increased even as high as $180 \mathrm{deg} / \mathrm{s}$ or more.

\subsubsection{Design, Hardware, and Testing}

Some of the limitations to the design presented in Chapter 4 included using springs to restore the fingers to an extended position. Some of the fingers did not extend as far as those anatomically. This could be remedied by increasing the spring stiffness or increasing the spring leg length to increase the restoring torque in extended positions. To improve flexion positions moving the cable further away from the center of the pins would cause a larger lever and easier to close the fingers. Additionally, the size of the fingers could be improved to allow larger flexion angles. A Bowden cable with a Teflon lining or material other than steel would have also reduced the friction within the tube and resulted in a higher efficiency. Regarding the hardware, a Raspberry Pi would have been a better choice compared to the Arduino Mega since the clock speed was $16 \mathrm{MHz}$ and 1.5 $\mathrm{GHz}$, respectively (this would more likely be a problem if used with the EMG signal and a speed test such as the SHAP) (Arduino Mega 2560 Rev3, 2020; Raspberry Pi 4 Tech Specs). 
As mentioned, the finger design partially attributes to the joints not cycling through their full ROM; however, the output signal was also subject to error. A more appropriate choice could have been to add potentiometers to the finger joints to measure the joint angles directly (Brenneis et al., 2017).

\subsection{Future Work}

In this thesis, movement was demonstrated in all 6 DOFs. With this success, the next step would be to control the device using a previously designed biomimetic controller relying on EMG signals as opposed to artificially created MATLAB input signals (Boots, 2019). The controller would have to be adapted to the DOFs unique to this prosthetic design, but that is well within its capabilities. Once the device was working with the biomimetic controller the next phase would be to utilize the prosthetic attachment pictured in Figure 4.1 (F) and test the device with an amputee. Beyond this dexterity and speed testing could occur with tests such as the Clothes Pin Test, Box and Blocks Test, or South Hampton Hand Assessment (SHAP) to assess and better modify the device. One potential way to scale this design to accommodate amputees of various arm sizes would be to use the morphometric/anthropomorphic tables. Some of these are detailed in Biomechanics and Moto Control of Human Movement by David A. Winter. These tables provide scaling factors that would allow the original model to be scaled using parameters such as age and weight. These values would have associated segment lengths for people who fit into those categories. Another goal would be to release this work as an open-source project to allow other people the ability to not only use this device but to experiment and explore other possible designs.

\subsection{Conclusions}

In this work, I was able to provide a solution for overcoming transradial prosthetic control limitations with additive manufacturing and modeling techniques. I was able to apply knowledge across a variety of engineering concepts including design, modeling, simulating, mechatronics, 
statistics, biomechanics, manufacturing, and more to simulate, design, and test a new 6-DOF device. Simulating the device focused on the torque at the two wrist DOFs: flexion-extension and pronation-supination to determine the appropriate hardware for realistic applications. The servos selected for the application were a $1.18 \mathrm{Nm}$ low-profile motor and a $1.96 \mathrm{Nm}$ standard motor for wrist pronation-supination and flexion-extension, respectively. Lower torque motors were selected as the experiment was focused on testing ROM and not focusing on weight/speed during the task. The servos selected would have been more than enough to cover a $1 \mathrm{~kg}$ object moving at $60 \mathrm{deg} / \mathrm{s}$. The next step was the physical design. The development consisted of many changes from the original modified device including the addition of a controller system, coupling the middle, ring, and small fingers, and adding an addition wrist DOF. These choices were made to create a device that provided as much function as possible without adding to the weight of the residual limb or extending beyond size/space restrictions in the arm. This was measured with the RMSE values calculated between the reference and response output joint angles. The hand was shown to be more accurate during the thumb, wrist, and most index finger joints. The delay was also smaller in the index, wrist, and some thumb joints. Consistently, the wrist was shown to both have small delays and minimal error. I was able to add a wrist DOF to an existing robotic arm and show the ability to perform movements in each DOF. 


\section{References}

Anatomy of the bones: Hand. (n.d.). [Photograph]. Retrieved February 28, 2020, from http://www.people.vcu.edu/ mhcrosthwait/clrs317web/boneanatomy.htm

Arduino Mega 2560 Rev3. (2020). Arduino. https://store.arduino.cc/usa/mega-2560-r3

Arm and shoulder bones anterior view and posterior view. (2018). [Web].

https://www.anatomynote.com/human-anatomy/skeleton-anatomy/arm-and-shoulderbones-anterior-view-and-posterior-view/

Autodesk Inventor (2019.5.1). (2019). [Computer software]. Autodesk.

Bair, M. M., \& Gondal, A. Z. (2019). Anatomy, shoulder and upper limb, forearm radius. https://www.ncbi.nlm.nih.gov/books/NBK544512/

BCcampus. (2013a). 8.2 Bones of the Upper Limb. In Anatomy and Physiology. OpenStax. https://opentextbc.ca/anatomyandphysiology/chapter/8-2-bones-of-the-upper-limb/

BCcampus. (2013b). 9.5 Types of Body Movements. In Anatomy and Physiology. OpenStax. https://opentextbc.ca/anatomyandphysiology/chapter/9-5-types-of-body-movements/

Belter, J. T., \& Dollar, A. M. (2011). Performance characteristics of anthropomorphic prosthetic hands. 2011 IEEE International Conference on Rehabilitation Robotics, 1-7. https://doi.org/10.1109/ICORR.2011.5975476

Benz, H. L., Yao, J., Rose, L., Olgac, O., Kreutz, K., Saha, A., \& Civillico, E. F. (2016). Upper extremity prosthesis user perspectives on unmet needs and innovative technology. Conference Proceedings : ... Annual International Conference of the IEEE Engineering in Medicine and Biology Society. IEEE Engineering in Medicine and Biology Society. Annual Conference, 287-290. https://doi.org/10.1109/EMBC.2016.7590696 
Bertels, T., Schmalz, T., \& Ludwigs, E. (2009). Objectifying the functional advantages of prosthetic wrist flexion. JPO Journal of Prosthetics and Orthotics, 21(2), 74-78. https://doi.org/10.1097/JPO.0b013e3181a10f46

Biddiss, E., McKeever, P., Lindsay, S., \& Chau, T. (2011). Implications of prosthesis funding structures on the use of prostheses: Experiences of individuals with upper limb absence. Prosthetics and Orthotics International, 35(2), 215-224. https://doi.org/10.1177/0309364611401776

Boots, M. T. (2019). A Biomimetic Approach to Controlling Restorative Robotics [Dissertation]. West Virginia University.

Bordoni, B., \& Varacallo, M. (2019). Anatomy, Tendons. StatPearls Publishing. https://www.ncbi.nlm.nih.gov/books/NBK513237/

Bowden cable. (2012, November 26). Grace’s Guide. https://www.gracesguide.co.uk/Bowden_Cable

Brenneis, D. J. A., Dawson, M. R., \& Pilarski, P. M. (2017, August 15). Development of the HANDI hand: An inexpensive, multi-articulating, sensorized hand for machine learning research in myoelectric control. Myoelectric Controls Symposium, Fredericton, New Brunswick. https://sites.ualberta.ca/ pilarski/docs/papers/Brenneis_2017_MEC_preprint.pdf Cordella, F., Ciancio, A. L., Sacchetti, R., Davalli, A., Cutti, A. G., Guglielmelli, E., \& Zollo, L. (2016). Literature review on needs of upper limb prosthesis users. Frontiers in Neuroscience, 10(209), 1-14. https://doi.org/10.3389/fnins.2016.00209 
Crisco, J. J., Heard, W. M. R., Rich, R. R., Paller, D. J., \& Wolfe, S. W. (2011). The mechanical axes of the wrist are oriented obliquely to the anatomical axes. The Journal of Bone and Joint Surgery. American Volume., 93(2), 169-177. https://doi.org/10.2106/JBJS.I.01222

Cuellar, J. S., Smit, G., Zadpoor, A. A., \& Breedveld, P. (2018). Ten guidelines for the design of non-assembly mechanisms: The case of 3D-printed prosthetic hands. Proceedings of the Institution of Mechanical Engineers, Part H: Journal of Engineering in Medicine, 232(9), 962-971. https://doi.org/10.1177/0954411918794734

Dalley, S. A., Wiste, T. E., Withrow, T. J., \& Goldfarb, M. (2009). Design of a multifunctional anthropomorphic prosthetic hand with extrinsic actuation. IEEE/ASME Transactions on Mechatronics, 14(6), 699-706. https://doi.org/10.1109/TMECH.2009.2033113

Deijs, M., Bongers, R. M., Ringeling - van Leusen, N. D. M., \& van der Sluis, C. K. (2016). Flexible and static wrist units in upper limb prosthesis users: Functionality scores, user satisfaction and compensatory movements. Journal of NeuroEngineering and Rehabilitation, 13(26), 1-13. https://doi.org/10.1186/s12984-016-0130-0

Delph, M. A., Fischer, S. A., Gauthier, P. W., \& Martinez Luna, C. H. (2012). Rehabilitation Robotics Glove (pp. 1-84). Worcester Polytechnic Institute. https://web.wpi.edu/Pubs/Eproject/Available/E-project-042512143542/unrestricted/Rehabilitative_Robotic_Glove_MQP.pdf

Edemekong, P. F., Bomgaars, D. L., Sukumaran, S., \& Levy, S. B. (2020). Activities of daily living. StatPearls Publishing. https://www.ncbi.nlm.nih.gov/books/NBK470404/ ElKoura, G., \& Singh, K. (2003). Handrix: Animating the human hand. The Eurographics Association, 1-11. 
Fan, S., Fan, S., Jiang, L., \& Liu, H. (2016). A design of a miniaturized prosthetic wrist based on repetition rate of human wrist daily tasks. 2016 IEEE International Conference on Robotics and Biomimetics (ROBIO), 1643-1648.

https://doi.org/10.1109/ROBIO.2016.7866563

Forro, S. D., \& Lowe, J. B. (2019). Anatomy, Shoulder and Upper Limb, Arm Structure and Function. StatPearls Publishing. https://www.ncbi.nlm.nih.gov/books/NBK507841/

Gaetani, F., Primiceri, P., Antonio Zappatore, G., \& Visconti, P. (2019). Hardware design and software development of a motion control and driving system for transradial prosthesis based on a wireless myoelectric armband. IET Science, Measurement Technology, 13(3), 354-362. https://doi.org/10.1049/iet-smt.2018.5108

Geethanjali, P. (2016). Myoelectric control of prosthetic hands: State-of-the-art review. Medical Devices (Auckland, N.Z.), 9, 247-255. https://doi.org/10.2147/MDER.S91102

Gibson, I., Rosen, D., \& Stucker, B. (2015). Additive Manufacturing Technologies (Second Edition). Springer New York. https://doi.org/10.1007/978-1-4939-2113-3

Häger-Ross, C., \& Schieber, M. H. (2000). Quantifying the independence of human finger movements: Comparisons of digits, hands, and movement frequencies. The Journal of Neuroscience, 20(22), 8542-8550. https://doi.org/10.1523/JNEUROSCI.20-2208542.2000

Hand \& Forearm. (n.d.). InMoov. Retrieved March 5, 2020, from http://inmoov.fr/gallery-v2/ Hibbeler, R. C. (2013). Three-Dimensional Kinetics of a Rigid Body. In Engineering Mechanics Dynamics (13th ed., pp. 579-629). Pearson Prentice Hall.

Important Concepts and Choices in Physical Simulation—MATLAB \& Simulink. (n.d.). MathWorks. Retrieved April 12, 2021, from 
https://www.mathworks.com/help/physmod/simscape/ug/important-concepts-andchoices-in-physical-simulation.html

Kanitz, G., Montagnani, F., Controzzi, M., \& Cipriani, C. (2018). Compliant prosthetic wrists entail more natural use than stiff wrists during reaching, not (necessarily) during manipulation. IEEE Transactions on Neural Systems and Rehabilitation Engineering, 26(7), 1407-1413. https://doi.org/10.1109/TNSRE.2018.2847565

Kilbreath, S. L., \& Gandevia, S. C. (1994). Limited independent flexion of the thumb and fingers in human subjects. The Journal of Physiology, 479(3), 487-497. https://doi.org/10.1113/jphysiol.1994.sp020312

Krausz, N. E., Rorrer, R. A. L., \& Weir, R. F. ff. (2016). Design and fabrication of a six degreeof-freedom open source hand. IEEE Transactions on Neural Systems and Rehabilitation Engineering, 24(5), 562-572. https://doi.org/10.1109/TNSRE.2015.2440177

Kyberd, P. J. (2012). The influence of passive wrist joints on the functionality of prosthetic hands. Prosthetics and Orthotics International, 36(1), 33-38. https://doi.org/10.1177/0309364611426905

Kyberd, P. J., Lemaire, E. D., Scheme, E., MacPhail, C., Goudreau, L., Bush, G., \& Brookeshaw, M. (2011). Two-degree-of-freedom powered prosthetic wrist. The Journal of Rehabilitation Research and Development, 48(6), 609. https://doi.org/10.1682/JRRD.2010.07.0137

Maat, B., Smit, G., Plettenburg, D., \& Breedveld, P. (2018). Passive prosthetic hands and tools: A literature review. Prosthetics and Orthotics International, 42(1), 66-74. https://doi.org/10.1177/0309364617691622 
Mandich, M. B. (2015). Development in the Preschool Years. In A. Cronnin (Ed.), Human Development and Performance Throughout the Life Span (2nd ed., pp. 260-261). Cenegage Learning.

Mason, C. R., Gomez, J. E., \& Ebner, T. J. (2001). Hand synergies during reach-to-grasp. Journal of Neurophysiology, 86(6), 2896-2910. https://doi.org/10.1152/jn.2001.86.6.2896

Material extrusion. (n.d.). Retrieved December 1, 2020, from http://seb199.me.vt.edu/dreams/material-extrusion/

MATLAB (9.9.0.1538559 (R2020b)). (n.d.). [Computer software]. The MathWorks Inc.

Meetze, S. (2020). Normally-closed flexy-hand terminal device 1.0. YouMagine. https://www.youmagine.com/designs/normally-closed-flexy-hand-terminal-device-1-0

Mell, A. G., Childress, B. L., \& Hughes, R. E. (2005). The effect of wearing a wrist splint on shoulder kinematics during object manipulation. Archives of Physical Medicine and Rehabilitation, 86(8), 1661-1664. https://doi.org/10.1016/j.apmr.2005.02.008

Montagnani, F., Controzzi, M., \& Cipriani, C. (2016). Independent long fingers are not essential for a grasping hand. Scientific Reports, 6(1), 1-9. https://doi.org/10.1038/srep35545

National Academies of Sciences, Engineering, and Medicine. (2017). The Promise of Assistive Technology to Enhance Activity and Work Participation (p. 24740). National Academies Press. https://doi.org/10.17226/24740

Neumann, D. A. (2017). Wrist. In Kinesiology of the Musculoskeletal System: Foundataions for Rehabilitation (3rd ed., p. 228). Elsevier.

Nycz, C. J., Bützer, T., Lambercy, O., Arata, J., Fischer, G. S., \& Gassert, R. (2016). Design and characterization of a lightweight and fully portable remote actuation system for use with 
a hand exoskeleton. IEEE Robotics and Automation Letters, 1(2), 976-983. https://doi.org/10.1109/LRA.2016.2528296

Olsen, N. R., George, J. A., Brinton, M. R., Paskett, M. D., Kluger, D. T., Tully, T. N., Duncan, C. C., \& Clark, G. A. (2019). An adaptable prosthetic wrist reduces subjective workload (pp. 1-19) [Preprint]. Bioengineering. https://doi.org/10.1101/808634

Ottobock. (2015). Myoelectric compared to body-powered prostheses. Otto Bock Clinical Research \& Services. https://handsmartgroup.org/wpcontent/uploads/2012/10/Myoelectric-compared-to-body-powered-prostheses-studysummaries.pdf

Pasquina, P. F., Perry, B. N., Miller, M. E., Ling, G. S. F., \& Tsao, J. W. (2015). Recent advances in bioelectric prostheses. Neurology: Clinical Practice, 5(2), 164-170. https://doi.org/10.1212/CPJ.0000000000000132

Ramirez, J., Rubiano, A., \& Castiblanco, P. (2019a). Soft driving epicyclical mechanism for robotic finger. Actuators, 8(3), 58. https://doi.org/10.3390/act8030058

Ramirez, J., Rubiano, A., \& Castiblanco, P. (2019b). Soft driving epicyclical mechanism for robotic finger: Pulley/servo (No. 3) [Photograph]. Multidisciplinary Digital Publishing Institute. https://www.mdpi.com/2076-0825/8/3/58

Raspberry Pi 4 tech specs. (n.d.). Raspberry Pi. Retrieved June 29, 2020, from https://www.raspberrypi.org/products/raspberry-pi-4-model-b/specifications/

Reaz, M. B. I., Hussain, M. S., \& Mohd-Yasin, F. (2006). Techniques of EMG signal analysis: Detection, processing, classification and applications. Biological Procedures Online, 8(1), 11-35. https://doi.org/10.1251/bpo115 
Robertson, D. G. E., Caldwell, G. E., Hamill, J., Kamen, G., \& Whittlesey, S. N. (2013). ThreeDimensional Kinematics. In Research Methods in Biomechanics (2nd ed., pp. 37-54). Human Kinetics.

Semasinghe, C. L., Madusanka, D. G. K., Ranaweera, R. K. P. S., \& Gopura, R. A. R. C. (2019). Transradial prostheses: Trends in development of hardware and control systems. The International Journal of Medical Robotics and Computer Assisted Surgery, 15(1), e1960. https://doi.org/10.1002/rcs.1960

Sobinov, A. (2019). Description of Motor Control Using Inverse Models [Dissertation]. West Virginia University.

Steuernagel, W. (n.d.). Accounting for lost motion [White Paper]. Cable Manufacturing \& Assembly Co. Retrieved May 21, 2020, from https://www.cmacable.com/wpcontent/uploads/2014/05/accounting-for-lost-motion-whitepaper.pdf

The three anatomical planes of movement. (2016). [Photograph]. https://parallelcoaching.co.uk/what-are-the-three-anatomical-planes-of-movement

Timeline: Prosthetic limbs through the years. (2015, March 8). UPMC HealthBeat. https://share.upmc.com/2015/03/timeline-prosthetic-limbs-years/

Uellendahl, J. (2017). Myoelectric versus body-powered upper-limb prostheses: A clinical perspective. JPO: Journal of Prosthetics and Orthotics, 29(12), P25-P29. https://doi.org/10.1097/JPO.0000000000000151

Ultimate materials guide -tips for 3D printing with PLA. (2020). Simplify3D. /support/materialsguide/pla/

Ultimate materials guide-3D printing flexible filament. (2020). Simplify3D. /support/materialsguide/flexible/ 
Ultimate materials guide_-Tips for 3D printing with PETG. (2020). Simplify3D. /support/materials-guide/petg/

Weir, R. F. ff. (2005a). Design of Artificial Arms and Hands for Prosthetic Applications. In Standard Handbook of Biomedical Engineering (p. 32.1-32.61). McGraw-Hill. https://pdfs.semanticscholar.org/1d5e/f9e48b17f30cedd07cdd3f61f5474a80cb28.pdf?_ga $=2.11875892 .187076627 .1583347908-1314812481.1576423795$

Weir, R. F. ff. (2005b). Design of Artificial Arms and Hands for Prosthetic Applications. In Standard Handbook of Biomedical Engineering (p. 32.32). McGraw-Hill. https://pdfs.semanticscholar.org/1d5e/f9e48b17f30cedd07cdd3f61f5474a80cb28.pdf?_ga $=2.11875892 .187076627 .1583347908-1314812481.1576423795$

Which design? (2016, January 20). Enabling The Future. http://enablingthefuture.org/whichdesign/

Yough, M. G., Hardesty, R. L., Yakovenko, S., \& Gritsenko, V. (2021). A segmented forearm model of hand pronation-supination approximates joint moments for real time applications**Research supported by National Institute of General Medical Sciences and the National Institute of Child Health and Human Development. 2021 10th International IEEE/EMBS Conference on Neural Engineering (NER), 751-754.

https://doi.org/10.1109/NER49283.2021.9441405 


\section{Appendix A: Prosthetic Review}

\section{A.1: Commercial and Research Based Prosthetic Comparison}

This section of Appendix A contains two tables detailing the prosthetics that are commercially available as well as those that are still undergoing research.

Table 1. Commercial Prosthetic Comparison. This table contains a detailed comparison of different prosthetics currently on the market. The categories are broken down into type of actuation, control, fingers that are controllable, wrist DOFs that are controllable, whether or not it is $3 \mathrm{D}$ printed, and weight.

\begin{tabular}{|c|c|c|c|c|c|c|c|c|}
\hline Prosthetic Name & Actuation & Control & Finger Control & Wrist DOF & 3D Printed & Weight & Image & Resources \\
\hline UnLimbited Arm & BP: Elbow Driven & Elbow Driven & $\begin{array}{l}\text { C.: Thumb (1), } \\
\text { Index, Middle, } \\
\text { Ring, \& Pinky }\end{array}$ & None & Yes & $<350 \mathrm{~g}$ & & $\begin{array}{l}\text { (Team Unlimbited Arm, 2021) } \\
\text { (Team Unlimbited, 2018) } \\
\text { (Team_Unlimbited 2016) }\end{array}$ \\
\hline The Raptor Hand & BP: Wrist Driven & Wrist Driven & $\begin{array}{l}\text { C.: Thumb (1), } \\
\text { Index, Middle, } \\
\text { Ring, \& Pinky }\end{array}$ & None & Yes & N/A & & $\begin{array}{l}\text { (Dally et al., 2015) } \\
\text { (Walter, 2015) } \\
\text { (Kate et al., 2017) }\end{array}$ \\
\hline Luke Arm & EP: EMG & EMG & $\begin{array}{c}\text { I.: Thumb (2), } \\
\text { Index (1), Middle, } \\
\text { Ring, \& Pinky (1) }\end{array}$ & FE/RU (1), PS (1) & No & $1.4 \mathrm{~kg}$ & & \begin{tabular}{|l} 
(Mobius Bionics, n.d.) \\
(Mobius Bionics, 2021)
\end{tabular} \\
\hline Bebionic 3 & EP: EMG & EMG & $\begin{array}{l}\text { I.: Thumb (1), } \\
\text { Index(1), Middle } \\
\text { (1), Ring (1), \& } \\
\text { Pinky (1) }\end{array}$ & PS (1) & No & $\begin{array}{l}<600 \mathrm{~g} \\
\text { (hand } \\
\text { alone) }\end{array}$ & & \begin{tabular}{|l} 
(RSLSteeper, n.d.) \\
(Atzori \& Müller, 2015)
\end{tabular} \\
\hline Michelangelo Hand & EP: EMG & EMG & \begin{tabular}{|c|} 
I.: Thumb (1), \\
Index (1), Middle, \\
Ring, and Pinky (1)
\end{tabular} & FE (1), PS (1) & No & $\sim 510 \mathrm{~g}$ & & $\begin{array}{l}\text { (Atzori \& Müller, 2015) } \\
\text { (Ottobock, 2020) }\end{array}$ \\
\hline $\begin{array}{l}\text { i-Limb Quantum } \\
\text { Prosthetic Hand }\end{array}$ & EP: EMG & EMG & $\begin{array}{l}\text { I.: Thumb (2), } \\
\text { Index (1), Middle } \\
\text { (1), Ring (1), \& } \\
\text { Pinky (1) }\end{array}$ & FE (1), PS (1) & No & $<515 g$ & & $\begin{array}{l}\text { (Atzori \& Müller, 2015) } \\
\text { (Össur, n.d.) }\end{array}$ \\
\hline $\begin{array}{c}\text { TASKA Proshetic } \\
\text { Hand }\end{array}$ & EP: EMG & EMG & $\begin{array}{l}\text { I.: Thumb (2), } \\
\text { Index, Middle, } \\
\text { Ring, \& Pinky }\end{array}$ & FE,PS & No & N/A & & $\begin{array}{l}\text { (TASKA Prosthetics, 2017) } \\
\text { (Designers Institute of New } \\
\text { Zealand, 2018) }\end{array}$ \\
\hline Hero Arm & EP: EMG & EMG & $\begin{array}{l}\text { I.: Thumb (1), } \\
\text { Middle, Index } \\
\text { C.: Ring \& Pinky }\end{array}$ & N/A & No & $1 \mathrm{~kg}$ & & (Open Bionics, 2021) \\
\hline InMoove & EP: Microcontroller & Microcontroller & $\begin{array}{l}\text { I.: Thumb (2), } \\
\text { Index, Middle, } \\
\text { Ring, \& Pinky }\end{array}$ & PS & Yes & N/A & & (InMoov, n.d.) \\
\hline \multicolumn{9}{|c|}{$\begin{array}{l}\text { Notes: } \\
\mathrm{BP}=\text { body-powered, EP=externally-powered } \\
\text { I=independent finger control, C=coupled finger control } \\
\text { Thumb(1)-1 DOF, Thumb(2)-2-DOF } \\
\text { FE= flexion/extension, PS= pronation/supination, RU= radial/ulnar deviation }\end{array}$} \\
\hline
\end{tabular}


Table 2. Research based prosthetic comparison. This table details the type of actuation, control method, wrist DOF used, if it was manufactured using 3D printing, and the weight for some prosthetics undergoing research.

\begin{tabular}{|c|c|c|c|c|c|c|c|}
\hline Prosthetic Name & Actuation & Control & Wrist DOF & 3D Printed & Weight & Image & Resources \\
\hline SmartHand & EP & EMG & $\mathrm{N} / \mathrm{A}$ & No & $>675 g$ & & (Cipriani et al., 2011) \\
\hline 2-DOF Wrist & EP & $\begin{array}{l}\text { Switches } \\
\text { EMG }\end{array}$ & FE,PS & No & $200 \mathrm{~g}$ & & (Kyberd et al., 2011) \\
\hline Wrist Attachment & EP & Microcontroller & FE,PS,RU & Yes & $360 \mathrm{~g}$ & & (Olsen et al., 2019) \\
\hline Reachy & EP & EMG & FE,PS & Yes & $1.4 \mathrm{~kg}$ & & (Mick et al., 2019) \\
\hline N/A & EP & EMG & None & Yes & $250 \mathrm{~g}$ & & (Cognolato et al., 2018) \\
\hline $\begin{array}{l}\text { Rehabilitation } \\
\text { Robotic Glove }\end{array}$ & EP & $\begin{array}{c}\text { Switch } \\
\text { EMG } \\
\text { Programmed }\end{array}$ & None & No & $\begin{array}{l}\text { backpack: } \sim 6 \mathrm{~kg} \\
\quad \text { arm: } \sim 1 \mathrm{~kg}\end{array}$ & & (Delph et al., 2002) \\
\hline Adam's Arm & EP & EMG & FE, PS & Yes & N/A & & (Gaetani et al., 2019) \\
\hline
\end{tabular}




\section{A.2: Additional Resources}

This section contains the additional resources used to create the tables found in A.1.

[InMoov 3D printed robot hand and forearm] [Photograph] (2020). InMoov. http://inmoov.fr/gallery-v2/

[TASKA prosthetic hand] [Photograph]. 2018. Best Awards. https://bestawards.co.nz/product/consumer/4ormfunction/taska-prosthetics-prosthetichand/

[The UnLimbited Arm v2.1—Alfie Edition] [Photograph]. (2016). Thingiverse. https://www.thingiverse.com/thing:1672381

Atzori, M., \& Müller, H. (2015). Control capabilities of myoelectric robotic prostheses by hand amputees: a scientific research and market overview. Frontiers in Systems Neuroscience, 9(162), 1-7. https://doi.org/10.3389/fnsys.2015.00162

Cipriani, C., Controzzi, M., \& Carrozza, M. C. (2011). The SmartHand transradial prosthesis. Journal of NeuroEngineering and Rehabilitation, 8(1), 29. https://doi.org/10.1186/17430003-8-29

Cognolato, M., Atzori, M., Marchesin, C., Marangon, S., Faccio, D., Tiengo, C., Bassetto, F., Gassert, R., Petrone, N., \& Müller, H. (2018). Multifunction control and evaluation of a 3D printed hand prosthesis with the Myo armband by hand amputees [Preprint]. Bioengineering. https://doi.org/10.1101/445460

Dally, C., Johnson, D., Canon, M., Ritter, S., \& Mehta, K. (2015). Characteristics of a 3Dprinted prosthetic hand for use in developing countries. 2015 IEEE Global Humanitarian Technology Conference (GHTC), 66-70. https://doi.org/10.1109/GHTC.2015.7343956

Delph, Michael A., Fischer, Sarah A., Gauthier, Philip W., Martinez Luna, Carlos H. (2002). Rehabilitation robotics glove. Massachusetts, United States: Worcester Polytechnic institute. https://web.wpi.edu/Pubs/E-project/Available/E-project-042512143542/unrestricted/Rehabilitative_Robotic_Glove_MQP.pdf

Gaetani, F., Primiceri, P., Antonio Zappatore, G., \& Visconti, P. (2019). Hardware design and software development of a motion control and driving system for transradial prosthesis based on a wireless myoelectric armband. IET Science, Measurement Technology, 13(3), 354-362. https://doi.org/10.1049/iet-smt.2018.5108

Kate, J., Smit, G., \& Breedveld, P. (2017, February 2). 3D-printed upper limb prostheses: A review. Disability and Rehabilitation Assistive Technology, 12(3), 1-15. https://www.researchgate.net/publication/313274657_3Dprinted_upper_limb_prostheses_a_review 
Kyberd, P. J., Lemaire, E. D., Scheme, E., MacPhail, C., Goudreau, L., Bush, G., \& Brookeshaw, M. (2011). Two-degree-of-freedom powered prosthetic wrist. The Journal of Rehabilitation Research and Development, 48(6), 609. https://doi.org/10.1682/JRRD.2010.07.0137

Mick, S., Lapeyre, M., Rouanet, P., Halgand, C., Benois-Pineau, J., Paclet, F., Cattaert, D., Oudeyer, P.-Y., \& de Rugy, A. (2019). Reachy, a 3D-printed human-like robotic arm as a testbed for human-robot control strategies. Frontiers in Neurorobotics, 13, 65. https://doi.org/10.3389/fnbot.2019.00065

Mobius Bionics. (2021). LUKE arm detail page - Mobius Bionics. Mobius Bionics. https://www.mobiusbionics.com/luke-arm/

Mobius Bionics. (n.d.). Luke arm system. Mobius Bionics. https://mobiusbionics.com/wpcontent/uploads/2017/08/Mobius-Bionics-LUKE-Product-Spec-Sheet.pdf

Olsen, N. R., George, J. A., Brinton, M. R., Paskett, M. D., Kluger, D. T., Tully, T. N., Duncan, C. C., \& Clark, G. A. (2019). An adaptable prosthetic wrist reduces subjective workload [Preprint]. Bioengineering. https://doi.org/10.1101/808634

Open Bionics. (2021). Hero Arm-User Guide. Open Bionics. Retrieved July 23, 2021, from https:/openbionics.com/hero-arm-user-guide/

Össur. (n.d.). I-Limb Quantum. Össur. Retrieved March 5, 2020, from https://www.ossur.com/en-us/prosthetics/arms/i-limb-quantum

Ottobock. (2020). Michelangelo prosthetic hand. Ottobock. Retrieved July 23, 2021, from https://www.ottobockus.com/prosthetics/upper-limb-prosthetics/solutionoverview/michelangelo-prosthetic-hand/

RSLSteeper. (n.d.). Bebionic3 technical information. Ottobock. https://shop.ottobock.ca/media/pdf/bebionic3_technical_information_-_Lo_Res.pdf

TASKA Prosthetics. (2017). Seven key features set TASKA apart. TASKA prosthetics. http://www.taskaprosthetics.com/the-taska/features/\#

Team Unlimbited [@TeamUnLimbited]. (2018, May 12). Full adult arm. Completed weight only 334g !!! [Tweet]. Retrieved March 5, 2020, from https://twitter.com/TeamUnLimbited/status/995358633272717312

Team Unlimbited Arm. (2021). Enabling the future. Retrieved July 23, 2021, from http://enablingthefuture.org/team-unlimbited-arm/

Team_Unlimbited. (2016, July 14). The UnLimbited Arm v2.1 - Alfie Edition. Thingiverse. thingiverse.com/thing:1672381

Walter. (2015). [Raptor reloaded 3D printed hand] [Photograph] Thrinter. http://thrinter.com/raptor-reloaded-print/ 


\section{Appendix B: Method for Calculating Analytical Model Solutions}

Appendix B contains additional steps that were sued to calculate the analytical solution for the model. To determine the analytical torque at the flexion-extension and pronation-supination axes, the first step was to calculate the summation of forces about the $\mathrm{x}, \mathrm{y}$, and $\mathrm{z}$ axes (shown in eq. (13)).

$$
\begin{aligned}
& \sum \mathrm{F}_{\mathrm{x}}=\mathrm{m}\left(\mathrm{a}_{\mathrm{G}}\right)_{\mathrm{x}} \\
& \sum \mathrm{F}_{\mathrm{y}}=\mathrm{m}\left(\mathrm{a}_{\mathrm{G}}\right)_{\mathrm{y}} \\
& \sum \mathrm{F}_{\mathrm{z}}=\mathrm{m}\left(\mathrm{a}_{\mathrm{G}}\right)_{\mathrm{z}}
\end{aligned}
$$

The terms in Eq. 1-3 include $m$ which is the mass of the body and $\left(\mathrm{a}_{\mathrm{G}}\right)_{\mathrm{x}},\left(\mathrm{a}_{\mathrm{G}}\right)_{\mathrm{y}}$, and $\left(\mathrm{a}_{\mathrm{G}}\right)_{\mathrm{z}}$ are the accelerations in the respective $\mathrm{x}, \mathrm{y}$, and $\mathrm{z}$ directions (Hibbeler, 2013). The forces $\mathrm{F}_{\mathrm{x}}, \mathrm{F}_{\mathrm{y}}$, and $\mathrm{F}_{\mathrm{z}}$ have units of $\mathrm{N}$. 


\section{Appendix C: Establishing a Local Point in a Global CS Example}

\section{C.1: Local to Global CS}

An example of how to calculate points from local CS 6 to the Global CS is shown below. At the end of this Appendix, an example of the joint CS setup is also presented. Eq. (4) presents the overall equation for converting local coordinates into global coordinates. This was the same method as described in Robertson's Research Methods in Biomechanics.

$$
{ }^{u} \mathrm{P}={ }^{u} R^{6 * 6} \mathrm{P}+{ }^{G} P_{6, \text { origin }}
$$

To calculate the rotation matrix, the $\mathrm{x}, \mathrm{y}$, and $\mathrm{z}$ unit vectors along with their $\mathrm{i}, \mathrm{j}$, and $\mathrm{k}$ components were needed for both the local CS at 6 and the global CS, shown in eq. 5.

$$
{ }^{u} R^{6}=\left[\begin{array}{lll}
x_{u i}{ }^{*} x_{6 i} & x_{u j}{ }^{*} y_{6 i} & x_{u k}{ }^{*} z_{6 i} \\
y_{u i}{ }^{*} x_{6 j} & y_{u j}{ }^{*} y_{6 j} & y_{u k}{ }^{*} z_{6 j} \\
z_{u i}{ }^{*} x_{6 k} & z_{u j}{ }^{*} y_{6 k} & z_{u k}{ }^{*} z_{6 k}
\end{array}\right]
$$

The remaining terms ${ }^{\mathrm{G}} \mathrm{P}_{6 \text { origin }}$ and ${ }^{6} \mathrm{P}$ were calculated in eq. (6) and eq. (7), respectively using the global origin coordinates for marker 6 and the local $\mathrm{x}, \mathrm{y}, \mathrm{z}$ coordinates of point $7 \mathrm{~b}$ defined in CS 6.

$$
\begin{gathered}
{ }^{u} \mathrm{P}_{6, \text { origin }}=\left[\begin{array}{c}
\mathrm{x}_{6} \\
\mathrm{y}_{6} \\
\mathrm{z}_{6} \\
1
\end{array}\right] \\
{ }^{6} \mathrm{P}=\left[\begin{array}{c}
\mathrm{x}_{7 \mathrm{~b}} \\
\mathrm{y}_{7 \mathrm{~b}} \\
\mathrm{z}_{7 \mathrm{~b}} \\
1
\end{array}\right]
\end{gathered}
$$

Forming the homogenous transformation equation, shown in eq. (8), was the final step to solving for the global coordinates of point 7b, once eq. (4) was satisfied.

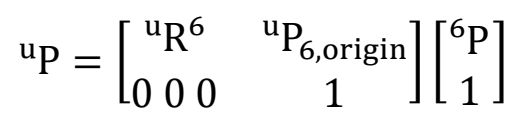

This process was repeated for the remaining four points listed above as well as for any other point calculated in another reference.

\section{C.2: Joint CS Setup}

This section details how the CS was setup for the example joint as well as how the method can be applied to calculate any joint.

With all the points globally defined, the CS at marker 7 was established by creating two vectors: one between 7 and $7 \mathrm{~b}$ and another between 7 and 7c. The $\mathrm{x}$ direction was assigned along $7 \rightarrow 7 \mathrm{~b}$, 
the $y$ vector was calculated perpendicular to the plane and the $\mathrm{z}$ direction was the cross product of vectors $\mathrm{x}$ and $\mathrm{y}$. From marker 7 the local distance to $8 \mathrm{~b}$ could be measured and a joint CS setup at point $7 \mathrm{~b}$ (between points 7b, 7c, and 8b). A local CS at the palm was also established using points $\mathrm{f} 1, \mathrm{f} 2$, and f3.

The local joint CS established at the index MCP joint and the segment CS setup at the palm will be used as an example to show the process by which the joint angles were calculated. The first step to determine the rotation angle was to obtain the rotation matrices, in Equation 5, for point f1 and point $7 b\left({ }^{u} R^{f 1}\right.$ ' and $\left.{ }^{u} R^{7 b}\right)$. The rotation sequence to obtain the correct order of frames is shown in Equation 9.

$$
{ }^{\mathrm{f} 1} \mathrm{R}^{7 \mathrm{~b}}={ }^{\mathrm{u}} \mathrm{R}^{\mathrm{f} 1{ }^{\prime} * \mathrm{u}^{\mathrm{u}}} \mathrm{R}^{7 \mathrm{~b}}
$$

This would rotate a frame from CS 7b to CS f1. 


\section{Appendix D: Simulation Verification}

This appendix contains the figures that verify the analytical and numerical calculations. 
A.

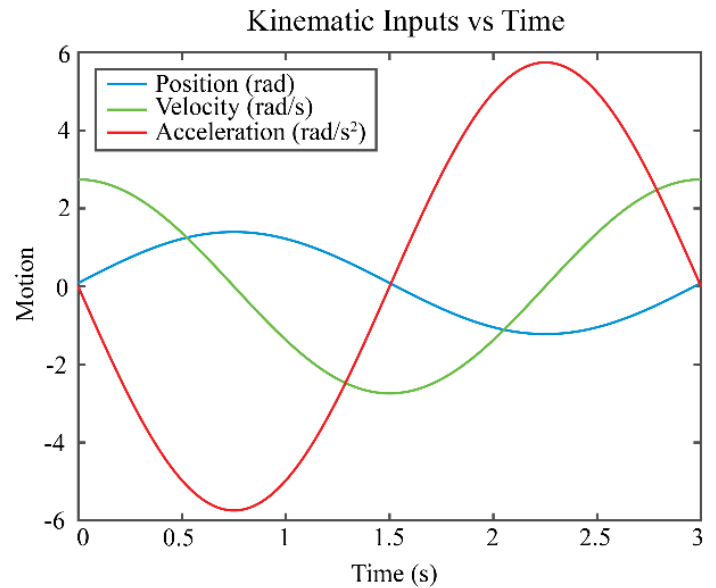

B.

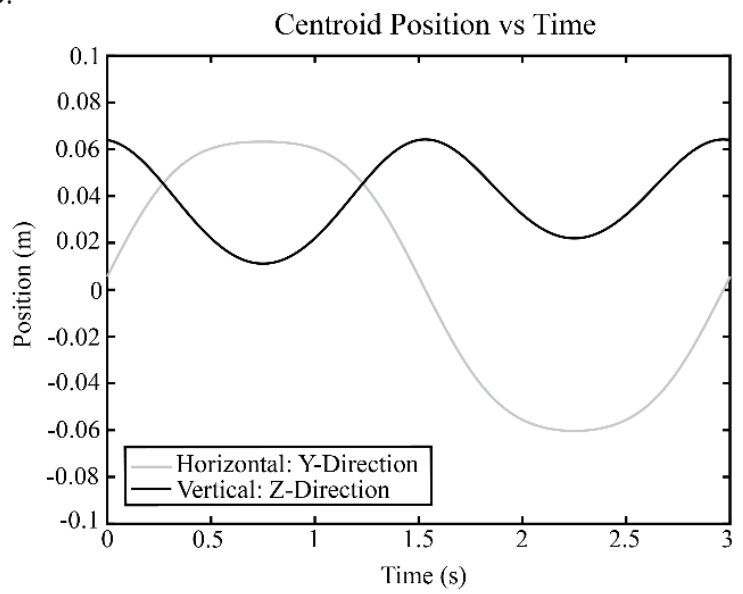

C.

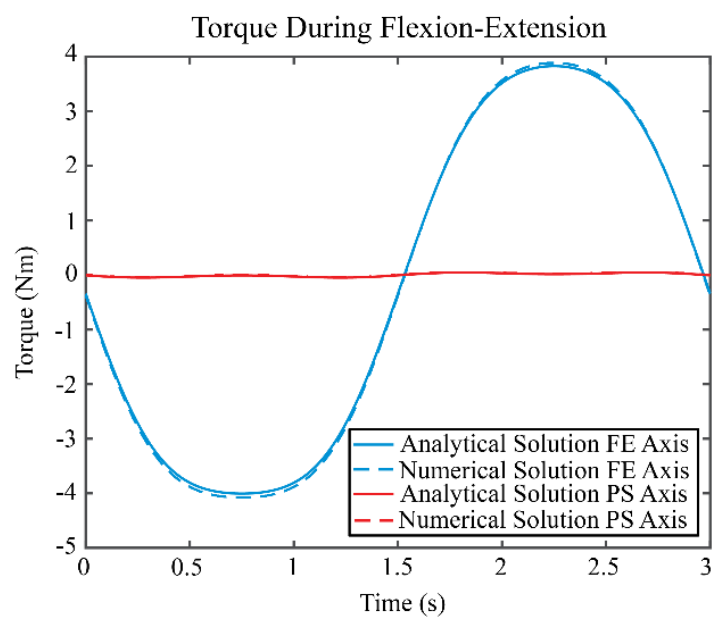

Figure 1. Wrist simulation verification for movement 1. Wrist flexion-extension, in a vertical position, with a weight of $6 \mathrm{~kg}$ moving at $120 \mathrm{deg} / \mathrm{s}$. A. Position, velocity, and acceleration during the simulation. B. Position in terms of horizontal and vertical movement directions. C. Torque comparison between the analytical and numerical solutions for both wrist flexion-extension and pronation-supination. 
A.

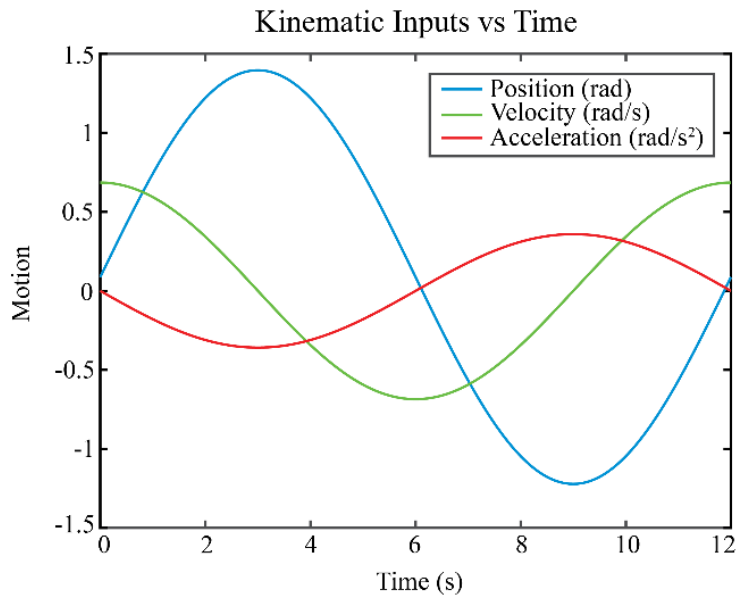

B.

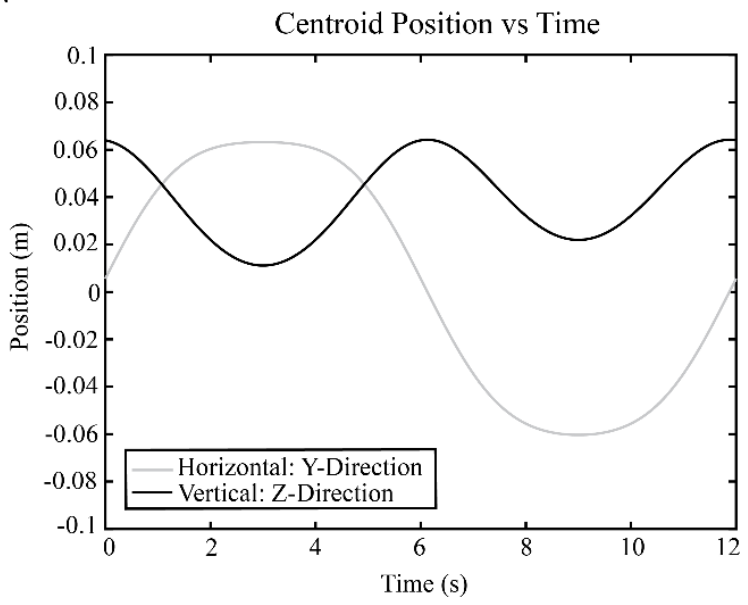

C.

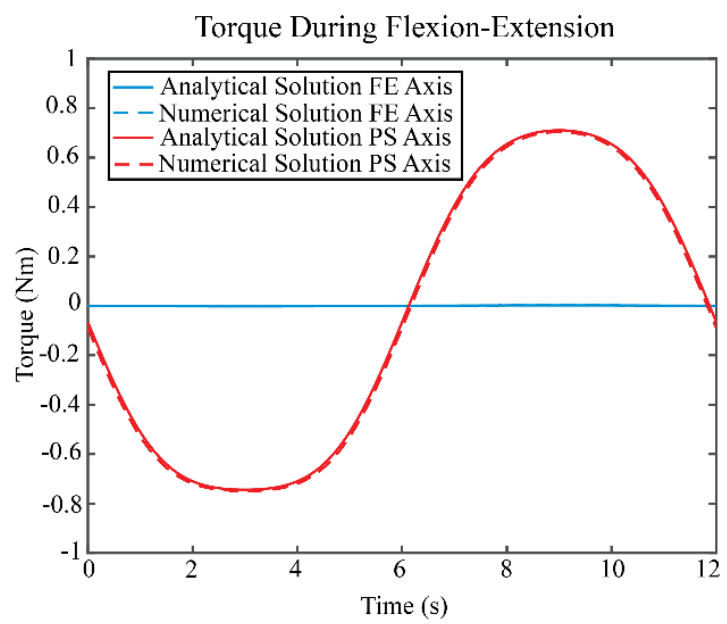

Figure 2. Wrist simulation verification for movement 2. Wrist flexion-extension, in a horizontal position, with a weight of $1 \mathrm{~kg}$ moving at $30 \mathrm{deg} / \mathrm{s}$. A. Position, velocity, and acceleration during the simulation. B. Position in terms of horizontal and vertical movement directions. C. Torque comparison between the analytical and numerical solutions for both wrist flexion-extension and pronation-supination. 
A.

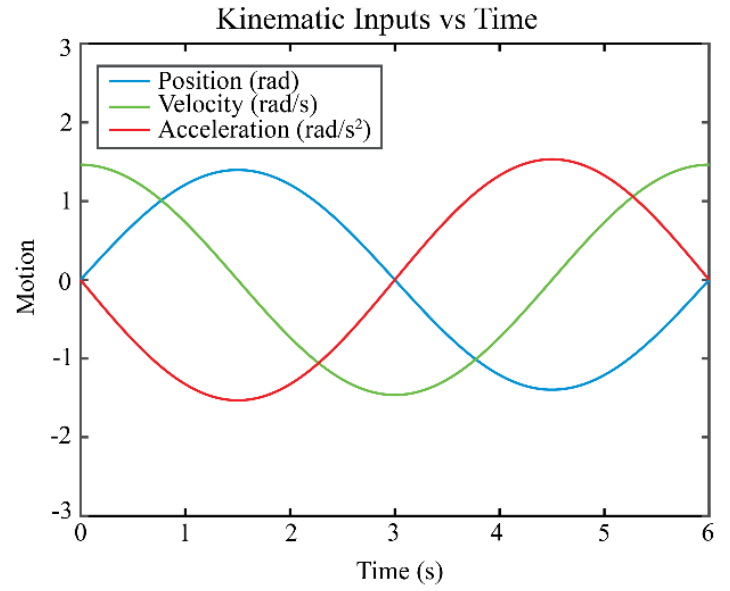

B.

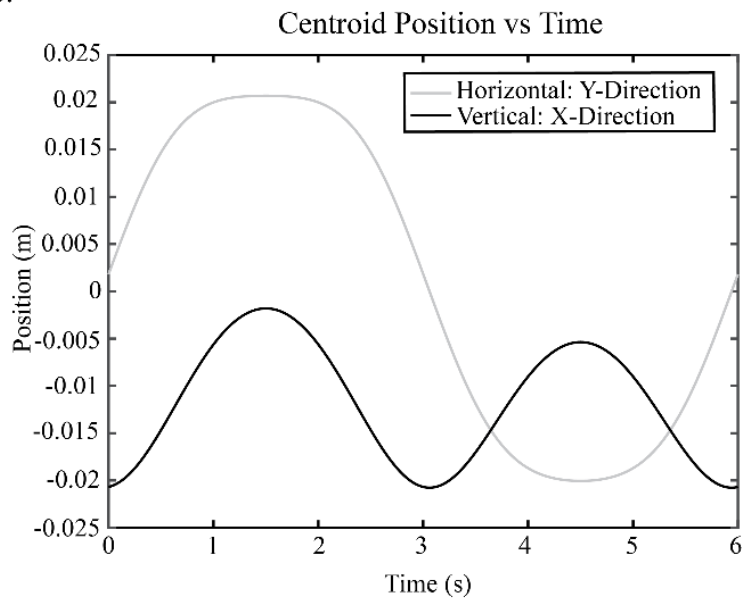

C.

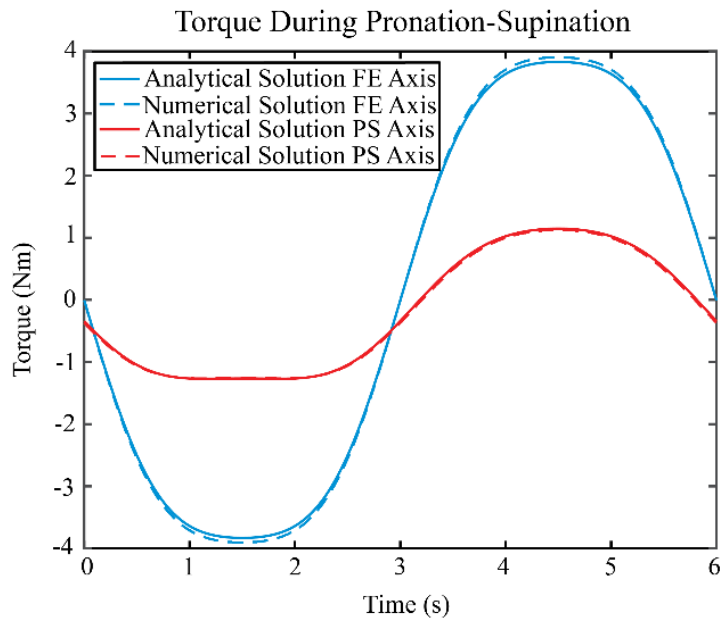

Figure 3. Wrist simulation verification for movement 3. Wrist pronation-supination, in a horizontal position, with a weight of $6 \mathrm{~kg}$ moving at $60 \mathrm{deg} / \mathrm{s}$. A. Position, velocity, and acceleration during the simulation. B. Position in terms of horizontal and vertical movement directions. C. Torque comparison between the analytical and numerical solutions for both wrist flexion-extension and pronation-supination. 